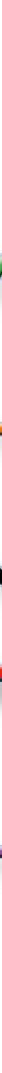

FAU Studies Mathematics \& Physics 13

Mathias Sirvent

Incorporating Differential Equations into

Mixed-Integer Programming for

Gas Transport Optimization 

Mathias Sirvent

Incorporating Differential Equations into

Mixed-Integer Programming for

Gas Transport Optimization 


\section{FAU Studies Mathematics \& Physics}

\section{Band 13}

Herausgeber der Reihe:

Prof. Dr. Karl-Hermann Neeb und Prof. Dr. Klaus Mecke 
Mathias Sirvent

\section{Incorporating Differential Equations into Mixed-Integer Programming for Gas Transport Optimization}

\section{Erlangen}

FAU University Press

2018 
Bibliografische Information der Deutschen Nationalbibliothek:

Die Deutsche Nationalbibliothek verzeichnet diese Publikation in der Deutschen Nationalbibliografie; detaillierte bibliografische Daten sind im Internet über http://dnb.d-nb.de abrufbar.

Autoren-Kontaktinformation: Mathias Sirvent, sirvent@posteo.de

Bitte zitieren als

M. Sirvent. Incorporating Differential Equations into Mixed-Integer Programming for Gas Transport Optimization. FAU Studies

Mathematics \& Physics Band 13. Erlangen: FAU University Press, 2018. DOI: 10.25593/978-3-96147-114-o.

Das Werk, einschließlich seiner Teile, ist urheberrechtlich geschützt. Die Rechte an allen Inhalten liegen bei ihren jeweiligen Autoren. Sie sind nutzbar unter der Creative Commons Lizenz BY-NC-ND.

Der vollständige Inhalt des Buchs ist als PDF über den OPUS Server der Friedrich-Alexander-Universität Erlangen-Nürnberg abrufbar: https://opus4.kobv.de/opus4-fau/home

Verlag und Auslieferung:

FAU University Press, Universitätsstraße 4, 91054 Erlangen

Druck: docupoint GmbH

ISBN: 978-3-96147-113-3 (Druckausgabe) eISBN: 978-3-96147-114-o (Online-Ausgabe)

ISSN: $\quad 2196-7482$

DOI: $\quad 10.25593 / 978-3-96147-114-0$ 


\title{
Incorporating Differential Equations into Mixed-Integer Programming for Gas Transport Optimization
}

\author{
Gemischt-Ganzzahlige Programmierung mit \\ Differentialgleichungen für die \\ Gastransportoptimierung
}

\author{
Der Naturwissenschaftlichen Fakultät \\ der Friedrich-Alexander-Universität Erlangen-Nürnberg
}

Zur

Erlangung des Doktorgrades Dr. rer. nat

vorgelegt von

Mathias Sirvent

aus Erlangen 
Als Dissertation genehmigt

von der Naturwissenschaftlichen Fakultät

der Friedrich-Alexander-Universität Erlangen-Nürnberg

Tag der mündlichen Prüfung: 29. März 2018

Vorsitzender des Promotionsorgans: Prof. Dr. Georg Kreimer

Gutachter:

Prof. Dr. Alexander Martin

Prof. Dr. Winnifried Wollner

Prof. Dr. Sven Leyffer 


\section{Acknowledgements}

I started my $\mathrm{PhD}$ thesis on April 15th, 2013. Exactly 4 years, 7 months, and 26 days later, the day has come. Today is December 11th, 2017 and I am grateful for an extremely interesting and educational period. I am happy to have the possibility to express sincere thanks to many people.

This thesis could not have been written without the support of my supervisor Prof. Dr. Alexander Martin. He introduced me to discrete optimization with the first lecture in 2010 and stimulated my enthusiasm for this research topic. After writing my master thesis in 2013, he gave me the chance to start as a $\mathrm{PhD}$ student at Friedrich-Alexander-Universität Erlangen-Nürnberg and I always felt supported and challenged by his guidance.

I wish to thank Prof. Dr. Martin Schmidt ${ }^{1}$ for his contribution on various joint papers, his strive for perfection, and his willingness to discuss our mathematical developments right down to the last detail. I learned a lot during the last years and this definitely has to do with his way of working.

I cannot thank Robert Burlacu ${ }^{1}$ enough for all the discussions on discrete optimization, the encouraging comments, and also the detection of wrongly spelled surnames. Moreover, I owe him the basic ideas for the example in Figure 4.14. Thanks Mr Burlesçu! In this context, I want to emphasize and appreciate the shared time with Denis Aßmann ${ }^{1}$, Julia Grübel, Andreas Heidt, Lena Hupp, Manu Kapolke ${ }^{1}$, and Galina Orlinskaya ${ }^{1}$.

Furthermore, I wish to express my thanks to Prof. Dr. Martin Gugat, Prof. Dr. Günter Leugering, David Wintergerst, and Prof. Dr. Winnifried Wollner for their support in our common papers and the great collaborations. Special thanks to Prof. Dr. Winnifried Wollner for the acceptance of being referee.

Additionally, I want to thank Nikolaos Kanelakis and Dr. Pandelis Biskas for our joint work on the Greek natural gas transport network; see Figure 4.16. My stay at the Aristotle University of Thessaloniki in 2016 was very pleasant and interesting.

\footnotetext{
${ }^{1}$ Thank you for proof-reading parts of this thesis.
} 
My next thanks go to Prof. Dr. Frauke Liers for maintaining a very enjoyable working atmosphere at our chair. Additionally, I want to thank her, Johann Schmitt, and Jeroen Stolwijk for the cooperation inside of the integrated graduate school of the academic research project "SFB Transregio 154: Mathematical Modeling, Simulation, and Optimization using the Example of Gas Networks". In this context, I want to thank the German Research Foundation (DFG) for the corresponding financial support, Marc Pfetsch for being my mentor inside of the program, and all the other doctoral students of the project that were not mentioned so far: Oliver Habeck, Christoph Huck, Andrés González, Thomas Kugler, Oliver Kunst, Björn Liljegren-Sailer, Michael Schuster, Pascal Mindt, Sabrina Nitsche, Tom Walther, and Dr. Tobias Wollenberg.

Many thanks go to all the remaining colleagues of the group: Dennis Adelhütte $^{1}$, Dr. Andreas Bärmann, Lucia Bäuml, Gabriela Bittner, Matej Ciesko, Thorsten Gellermann, Patrick Gemander, Dr. Christine Hayn, Beate Kirchner, Thomas Kleinert ${ }^{1}$, Vanessa Krebs ${ }^{1}$, Katja Kutzer, Dr. Maximilian Merkert, Dr. Antonio Morsi, Dr. Johannes Müller, Dr. Susanne Pape, Andrea Peter, Dr. Jakob Schelbert, PD Dr. Lars Schewe, Andreas Schmutzer, Oskar Schneider, Hanno Schülldorf, Johannes Thürauf ${ }^{1}$, Dr. Christoph Thurner ${ }^{1}$, Sebastian Tschuppik ${ }^{1}$, Christina Weber, and Dr. Dieter Weninger ${ }^{1}$. Special thanks to Dr. Björn Geißler and Dr. Martin Weibelzahl for the collaborations in our common papers.

Apart from our group, I wish to express my thanks to the professional table football crew of the 3rd and 4th floor: Dr. Martin Knossalla, Tobias Kufner, Björn Liljegren-Sailer, Fabian Rüffler, Johannes Semmler, Michele Spinola, Christoph Strohmeyer, Maximilian Walther, and David Wintergerst.

Furthermore, I appreciate the exchange of experiences with my friends that are or were heading for a $\mathrm{PhD}$ thesis in chemistry, computer science, economics, engineering, international business, or materials science: Marcus Becher, Dr. Anja Fingerhut, Micael Alonso Frank, Dr. Mario Heene, Sebastian Holzmann, Dr. Johannes Holzwarth, Thomas Matousek, Daniel Sekula, and Christian Sölch.

My special thanks to Mariana Sirvent, Ricardo Sirvent, and Maria Schütz ${ }^{1}$. Thanks for your patience, support, encouragement, and your belief in me.

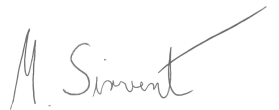

Mathias Sirvent 


\section{Zusammenfassung}

Erdgas ist eine der essentiellsten Energiequellen der Erde und der Transport durch Gasnetzwerke demzufolge eine wichtige Aufgabe. Die damit relevante Gastransportoptimierung gehört zu einer Kategorie von mathematischen Optimierungsproblemen, die sowohl diskrete Entscheidungen, als auch Differentialgleichungen beinhaltet. Erstere ergeben sich durch aktive Netzwerkelemente wie Ventile, Schieber oder Kompressoren, während zweitere das physikalische Verhalten von Erdgas abbilden. In der Gastransportoptimierung stehen somit gemischt-ganzzahlige und differentialgleichungsbeschränkte Optimierungsprobleme im Fokus. Der wissenschaftliche Beitrag dieser Dissertation, um solche Probleme zu lösen, hat zwei Komponenten.

Zunächst werden drei neue globale Algorithmen präsentiert. Typische Lösungsansätze transformieren die Differentialgleichungen, um lineare Nebenbedingungen zu erhalten. Dies ist nachvollziehbar, da die gemischtganzzahlige lineare Programmierung die vielversprechendste Version der gemischt-ganzzahligen Programmierung darstellt. Die neuen globalen Algorithmen in dieser Dissertation verlassen sich nicht auf eine solche Transformation und können mit weniger Informationen über die beteiligten Differentialgleichungen arbeiten. In einem iterativen Prozess werden gemischtganzzahlige lineare Programme und kleine nichtlineare Programme abwechselnd gelöst. Des Weiteren wird die Korrektheit sowie die endliche Terminierung der Algorithmen bewiesen und ein umfangreiches theoretisches Gerüst für die Nebenbedingungsannahmen vorgestellt. Die mathematischen und algorithmischen Entwicklungen erlauben die Lösung von stationären Gastransportproblemen mit gewöhnlichen Differentialgleichungen. Dazu werden vielversprechende numerische Resultate für das griechische Gastransportnetzwerk vorgestellt. Darüber hinaus ist der Weg für allgemeine simulationsbasierte Algorithmen geebnet.

Im Anschluss wird ein Instantansteuerungsalgorithmus für die transiente Gastransportoptimierung mit partiellen Differentialgleichungen vorgestellt. Ein neues und spezifisches Diskretisierungsschema wird entwickelt, welches die Benutzung von gemischt-ganzzahligen lineare Programmen innerhalb des 
Instantansteuerungsalgorithmus' erlaubt. Erneut zeigen vielversprechende numerische Resultate die Anwendbarkeit des Ansatzes. Diese Ergebnisse bereiten den Weg für weitere Forschung im Bereich der transienten Gasnetzoptimierung, welche in der Literatur aufgrund ihrer Schwierigkeit seltener behandelt wird. 


\section{Abstract}

Natural gas is one of the most important energy sources. Consequently, its transportation through gas networks is an essential task and gives rise to gas transport problems. Such optimization problems involve discrete decisions to switch network elements as valves, control valves, or compressor machines. Moreover, the physical behavior of natural gas is described by differential equations. Thus, when dealing with gas transport optimization, mixedinteger problems constrained by differential equations become relevant. The scientific contribution of this thesis to solve such problems is twofold.

First, three new global algorithms are presented. In general, a typical solution approach transforms the differential equations to linear constraints. This is reasonable as mixed-integer linear programming is the most successful instance of mixed-integer programming. The new global algorithms in this thesis do not rely on this transformation and can work with less information about the underlying differential equation constraints. In an iterative process, mixed-integer linear programs and small nonlinear programs are solved alternately and the correct and finite terminations of the algorithms are proven. An extensive theoretical framework that distinguishes the assumptions on the constraints is set up. The developments allow to solve stationary gas transport optimization problems with ordinary differential equations. In this sense, promising numerical results for the Greek natural gas transport network are shown. Furthermore, the way for more general simulation-based algorithms is paved.

Second, an instantaneous control algorithm for transient gas network optimization with partial differential equations is presented. A new and specific discretization scheme that allows to use mixed-integer linear programs inside of the instantaneous control algorithm is developed for the example of gas. Again, promising numerical results that illustrate the applicability of the approach are shown. These findings pave the way for more research in the field of transient gas network optimization, which, due to its hardness, is often disregarded in the literature. 



\section{List of Figures}

3.1 Gross power generation in Germany; see AG Energiebilanzen e.V. [1]. . . . . . . . . . . . . . . . . 27

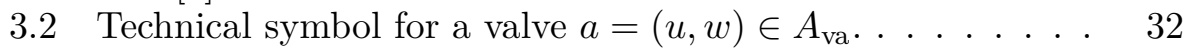

3.3 Technical symbol for a control valve $a=(u, w) \in A_{\mathrm{cv}}$. . . . 32

3.4 Technical symbol for a compressor machine $a=(u, w) \in A_{\mathrm{cm}} .33$

3.5 Characteristic diagram of a turbo compressor with operating range (blue). . . . . . . . . . . . . . . . 34

3.6 Domain of pressure and mass flux function (blue). . . . . 38

4.1 Nonconvex feasible set (blue) for Problem (4.3) neglecting integrality. . . . . . . . . . . . . . 45

4.2 Illustration of Definition 4.3.2 and Definition 4.3.3. . . . . 51

4.3 Bound tightening for $x_{i}$ with tightened box (blue). . . . . . 52

4.4 Setting for subproblem and master problem for Algorithm 4.1. 54

4.5 Illustration of the proof of Lemma 4.4.4 (b). . . . . . . 58

4.6 Illustration of the proof of Theorem 4.4.6. . . . . . . . 59

4.7 Illustration of the proof of Theorem 4.4.7 . . . . . . . . 61

4.8 Setting for subproblem and master problem for Algorithm 4.2. 64

4.9 Using subproblem $(\mathrm{S}(i, k))$ fails for Algorithm 4.2. . . . . 65

4.10 Illustration of the proof of Lemma 4.5.4 . . . . . . . . 69

4.11 Setting for subproblem and master problem for Algorithm 4.3. 73

4.12 Illustration of Example 4.12. . . . . . . . . . . . . 75

4.13 Pathological examples with high number of iterations. . . . 82

4.14 Algorithm 4.1 with additional dimension in (4.2c): $x_{i_{3}}=5\left(\log \left(x_{i_{1}}\right)+\log \left(x_{i_{2}}\right)\right) \ldots \ldots \ldots \ldots . \ldots \ldots$

4.15 Classification of our stationary pipe model (4.33) in dark gray. 86

4.16 Greek natural gas transport network. . . . . . . . . . . 91

4.17 Subproblem averages over iterations for Algorithm 4.1. . . . 94

4.18 Subproblem boxplots over iterations for Algorithm 4.1. . . . 94

4.19 Master problem averages over iterations for Algorithm 4.1. . 95

4.20 Subproblem averages over iterations for Algorithm 4.2 and Algorithm 4.3. . . . . . . . . . . . . . . 97

4.21 Subproblem boxplots over selected iterations for Algorithm 4.2. 97 
4.22 Master problem averages over iterations for Algorithm 4.2 and Algorithm 4.3. . . . . . . . . . . . . 98

4.23 Tightened relaxation of in- and outflow pressure over the course of iterations. . . . . . . . . . . . 100

5.1 Classification of our transient pipe model (5.11) in dark gray. 111

5.2 Overview of the time discretizations for (5.11) . . . . . 112

5.3 Network of Testset One. . . . . . . . . . . . . . . . . . . 121

5.4 Profiles over the pipe's length for different time points. . . . 121

5.5 Network of Testset Two. . . . . . . . . . . . . . . . . . . 122

5.6 Profiles over the time horizon at entries, exists, and the flexible node. . . . . . . . . . . . . . . . . . 124

5.7 Pressure difference profile over the time horizon at active elements. . . . . . . . . . . . . . 125

5.8 Pressure difference profile (initial vs. target) over time for three time blocks. . . . . . . . . . . . . . 126

5.9 Gurobi's running times for solving all time-discrete MIPs. . 127 


\section{List of Tables}

4.1 Overview of the results of Algorithm 4.1. . . . . . . . . 93

4.2 Overview of the results of Algorithm 4.2 and Algorithm 4.3. 96 



\section{Contents}

Acknowledgements $\quad 1$

Zusammenfassung 3

Abstract 5

$\begin{array}{lc}\text { List of Figures } & 8\end{array}$

List of Tables $\quad 9$

1 Introduction 13

1.1 The Title of this Thesis . . . . . . . . . . . . 13

1.2 Challenges and Guidelines . . . . . . . . . . . . . . . 14

1.3 Scientific Contribution and Results . . . . . . . . . . . 15

1.4 Incorporation of Joint Work with other Authors . . . . . . . 16

1.5 Organization . . . . . . . . . . . . 16

2 Literature Survey: Basic Concepts 19

2.1 Computational Complexity . . . . . . . . . . . . . . 19

2.2 Linear Programming . . . . . . . . . . . . . . . . . . . 20

2.3 Mixed-Integer Linear Programming . . . . . . . . . . . . . . 21

2.4 Mixed-Integer Nonlinear Programming . . . . . . . . . . . 23

3 Gas Transport Optimization 27

3.1 The German Energy Industry . . . . . . . . . . . . . . . . 28

3.2 Modeling Gas Networks . . . . . . . . . . . . . . . . . 30

3.2 .1 Nodes . . . . . . . . . . . . . . . . . . . 31

$3.2 .2 \quad$ Arcs . . . . . . . . . . . . . . . . . . 32

3.2 .3 Summary . . . . . . . . . . . . . . . . . 39

3.3 Literature Survey: Solving Gas Transport Optimization Prob-

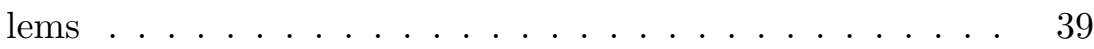


4 Decomposition Methods for Mixed-Integer Nonlinear $\begin{array}{ll}\text { Programs } & 43\end{array}$

4.1 Problem Statement . . . . . . . . . . . . . . . . . . . 44

4.1.1 General Mixed-Integer Nonlinear Program . . . . . . 44

4.1.2 Reformulated Mixed-Integer Nonlinear Program . . 44

4.2 The Assumptions . . . . . . . . . . . . . . . . . . 47

4.3 Basic Algorithmic Framework . . . . . . . . . . . . . . 48

4.4 The Case of Strictly Monotonic and Strictly Concave or Convex Functions . . . . . . . . . . . . . . . . . . . 51

4.4.1 Derivation of the Algorithm . . . . . . . . . . . 51

4.4.2 Convergence Results . . . . . . . . . . . . . . 56

4.5 The Case of Lipschitz Continuous Functions and Exact Function Evaluations . . . . . . . . . . . . 62

4.5.1 Derivation of the Algorithm . . . . . . . . . . . 62

4.5.2 Convergence Results . . . . . . . . . . . . . . 68

4.6 The Case of Lipschitz Continuous Functions and Inexact Function Evaluations . . . . . . . . . . . . . 72

4.6.1 Derivation of the Algorithm . . . . . . . . . . . 72

4.6 .2 Convergence Results . . . . . . . . . . . . . . 75

4.7 Literature Survey: Related Algorithms . . . . . . . . . . . . 78

4.8 Further Remarks . . . . . . . . . . . . . . . . . . . 80

4.8.1 Algorithmic Details . . . . . . . . . . . . . 80

4.8.2 Limitations and Extensions . . . . . . . . . . 82

4.9 Application to Stationary Gas Transport Optimization . . . 84

4.9 .1 Objective Function . . . . . . . . . . . . . 85

4.9 .2 Stationary Pipe Model . . . . . . . . . . . . . . . 85

4.9 .3 Numerical Results . . . . . . . . . . . . . . . . . . 89

5 Instantaneous Control for PDE-Constrained Mixed$\begin{array}{ll}\text { Integer Programs } & 103\end{array}$

5.1 Problem Statement and Derivation of the Algorithm . . . . 104

5.2 Literature Survey: Instantaneous Control . . . . . . . . . 105

5.3 Further Remarks . . . . . . . . . . . . . . . . . . . 106

5.4 Application to Transient Gas Transport Optimization . . . 106

5.4 .1 Objective Function . . . . . . . . . . . . . . 107

5.4 .2 Transient Pipe Model . . . . . . . . . . . . . . . . . 109

5.4 .3 Numerical Results . . . . . . . . . . . . . . . . . 120

6 Summary and Outlook $\quad 129$

$\begin{array}{ll}\text { Bibliography } & 131\end{array}$ 


\section{Introduction}

Mathematical programming is an important tool for modeling real-world problems from a large variety of applications like, e.g., engineering, economics, or the natural sciences. In this introduction, we define the general mathematical problem and introduce the considered application. Moreover, we state the challenges and point out the scientific achievements.

The introduction is structured as follows. We motivate and explain the title of the thesis in detail in Section 1.1. Section 1.2 states the challenges and introduces the guidelines that repeatedly emerge in the course of this study. Afterward, Section 1.3 underlines the scientific contributions and highlights results before Section 1.4 emphasizes the collaborative works that are incorporated into this thesis. Finally, Section 1.5 gives an overview on the following chapters.

\subsection{The Title of this Thesis}

Mathematical programming deals with maximizing or minimizing a function where the values of the involved variables are restricted by constraints. Such a problem is called mathematical program (MP) and many real-world problems can be modeled as MPs. Consequently, a successful strategy for mathematical programming is crucial. This strategy depends on the properties of the MP.

A decisive distinction for MPs is made when the involved variables are not only continuous but integer variables as well. We talk about mixedinteger programming in this case. These integers are typically relevant for problems where variables represent quantities that can only be integer-it is not possible to build 1.3 airplanes - and where variables represent decisions modeled by 0 and 1 . Thus, we define our general mathematical programming problem under consideration:

$$
\begin{array}{ll}
\min _{x} & h(x) \\
\text { s.t. } & A x \geq b, \quad x^{-} \leq x \leq x^{+}, \quad x_{\mathcal{C}} \in \mathbb{R}^{|\mathcal{C}|}, \quad x_{\mathcal{I}} \in \mathbb{Z}^{|\mathcal{I}|}, \\
& x_{\mathcal{C}} \in \mathcal{X} .
\end{array}
$$




\section{Introduction}

The sets $\mathcal{C}$ and $\mathcal{I}$ are the finite index sets of continuous and integer variables, respectively. Furthermore, the variable vector

reads $x:=\left(x_{\mathcal{C}}^{\top}, x_{\mathcal{I}}^{\top}\right)^{\top} \in \mathbb{R}^{|\mathcal{C}|} \times \mathbb{Z}^{|\mathcal{I}|}$ with bounds $x^{-} \leq x \leq x^{+}$. Linear constraints are given by $A x \geq b$ with $A \in \mathbb{R}^{m \times n}, n=n_{\mathcal{C}}+n_{\mathcal{I}}=|\mathcal{C}|+|\mathcal{I}|$, and $b \in \mathbb{R}^{m}$. A nonlinear objective is defined as $h: \mathbb{R}^{n} \rightarrow \mathbb{R}$. Finally, abstract constraints $x_{\mathcal{C}} \in \mathcal{X}$ are given.

Many real-world problems are governed by differential equations. Our application is gas transport optimization where the behavior of natural gas is described by the Euler equations, which are a set of hyperbolic partial differential equations. In our general mathematical programming problem (1.1), these differential equations are incorporated by (1.1c) in an abstract way. Additionally, gas transport optimization comprises decision variables to switch on and off active elements, e.g., valves, control valves, or compressor machines. This includes mixed-integer programming into our application.

Taken all together, the ingredients for the title of this thesis are available: Incorporating Differential Equations into Mixed-Integer Programming for Gas Transport Optimization.

\subsection{Challenges and Guidelines}

We want to solve Problem (1.1) and the challenging part emerges from the discrete aspects and from the differential equations incorporated by (1.1c). These equations are given as partial differential equations (PDEs) for transient gas transport optimization or as simplified ordinary differential equations (ODEs) for stationary gas transport optimization.

Mixed-integer programs including differential equations are typically solved by remodeling the differential equations to obtain an a-priori mixedinteger nonlinear or linear surrogate model. These remodeling steps are often subject to various simplification steps that are necessary to transform the differential equations into analytical functions. Our first challenge is to omit these simplification steps and develop general algorithms without relying on analytical functions. Moreover, we want to use the algorithms to solve stationary gas transport optimization problems that are constrained by ODEs.

Furthermore, transient gas transport optimization often leads to huge mathematical programs after remodeling. In general, these problems cannot be solved when the size of the networks are of practical size. Our second 
challenge is to find formulations and corresponding algorithms that are able to tackle transient gas transport optimization problems.

Note that the first challenge seeks for general algorithms that are then used for stationary gas transport optimization, whereas the second challenge is tailored to the application of transient gas transport optimization.

Our roadmap to cope with the challenges is inspired by two guidelines. We want to decompose our problems and we want to use mixed-integer linear programs. The simple reason for the first point is that decomposing problems into smaller ones has a successful history and is common sense for solving big problems. Second, mixed-integer linear programs underwent an enormous amount of research in the last decades. Consequently, fast, stable, robust, and reliable solvers as Gurobi, Cplex, or Scip are available and we use these solvers, in our case Gurobi, as a workhorse; see Gurobi [80], Cplex [36], and Maher et al. [110], respectively.

\subsection{Scientific Contribution and Results}

For the first challenge, we consider the case where the constraint functions in (1.1c) are not given analytically. We build up a new hierarchy of assumptions that restricts the knowledge about the nonlinear functions. Depending on the assumptions, we develop three new global decomposition algorithms that decompose the difficult constraints (1.1c), i.e., we rely on our first guideline. Specifically, we build upon relaxation strategies that are motivated by developments in the field of (mixed-integer) (non)linear programming and Lipschitz optimization. As a result, mixed-integer linear programs according to our second guideline are obtained. We prove correct and finite terminations and clarify the limitations of our approaches. Note that the presented framework is a general approach for problems in the form of (1.1). In particular, we consider stationary gas transport optimization, i.e., Problem (1.1) with the Euler equations in the form of ODEs in (1.1c). Moreover, we show promising numerical results for the real-world gas network of Greece.

Additionally, we consider the second challenge of transient gas transport optimization, i.e., Problem (1.1) with PDEs (1.1c). We use an instantaneous control algorithm and adapt it to the Euler equations for the first time. Specifically, we decompose the problem into single time steps and present new discretization techniques that ensure the mixed-integer linear program property. In other words, we build upon our guidelines again. Finally, we 


\section{Introduction}

show promising numerical results that illustrate the applicability of the approach.

\subsection{Incorporation of Joint Work with other Authors}

Some parts of this thesis incorporate collaborative work with other authors. These works are accepted for publication in international journals or are submitted for publication:

[76] M. Gugat, G. Leugering, A. Martin, M. Schmidt, M. Sirvent, and D. Wintergerst. "MIP-Based Instantaneous Control of Mixed-Integer PDE-Constrained Gas Transport Problems." In: Computational Optimization and Applications 70.1 (May 2018), pp. 267-294. DOI: 10.1007/s10589-017-9970-1.

[77] M. Gugat, G. Leugering, A. Martin, M. Schmidt, M. Sirvent, and D. Wintergerst. "Towards Simulation Based Mixed-Integer Optimization with Differential Equations." In: Networks (2018). DOI: 10.1002/net.21812.

[141] M. Schmidt, M. Sirvent, and W. Wollner. A Decomposition Method for MINLPs with Lipschitz Continuous Nonlinearities. Tech. rep. July 2017. URL: http://www.optimization-online.org/DB_ HTML/2017/07/6130.html (visited on 04/27/2018). Submitted to Mathematical Programming.

Within these joint works, we remark that the author of this thesis made significant contributions for conceiving, developing, and elaborating all the presented contents that follow. In addition, we explicitly state results that originate from co-authors and where the author of this thesis did not contribute.

\subsection{Organization}

Chapter 2 is a literature survey. It explains various specifications of Problem (1.1), their complexity, and how to solve them. The referred methods and algorithms are the foundation for the subsequent developments.

In Chapter 3, we specify our application of gas transport optimization and introduce the model in detail. Furthermore, we refer to existing algorithms and methods. 
In Chapter 4, we present three new and general decomposition algorithms for Problem (1.1) and prove their correct and finite terminations. In this context, we restrict the knowledge about the constraint in (1.1c) to address the case of functions that are not analytically given. Finally, we show promising numerical results for stationary gas transport optimization. Chapter 4 addresses the first challenge.

In Chapter 5, we introduce an instantaneous control algorithm for Problem (1.1) and break new ground by adapting it to transient gas transport optimization. Finally, we show promising numerical results. Chapter 5 addresses the second challenge.

In Chapter 6, we first give a summary by taking up the challenges defined in Section 1.2. In particular, we lay emphasize on the scientific contributions that are made in Chapter 4 and Chapter 5. Eventually, we give an outlook for future research. 



\section{Literature Survey: Basic Concepts}

The presented ideas in Chapter 4 and Chapter 5 build upon state-of-the-art algorithms for linear programs (LPs), mixed-integer linear programs (MIPs), and mixed-integer nonlinear programs (MINLPs). We briefly describe the main aspects of computational complexity theory in Section 2.1. Afterward, we introduce the main concepts for solving LPs, MIPs, and MINLPs in Section 2.2, Section 2.3, and Section 2.4, respectively.

\subsection{Computational Complexity}

Arora and Barak [4] provide all relevant information about computational complexity. In this chapter, we describe the important concepts to classify the problems that show up in this thesis.

An algorithm is a finite procedure that returns a correct answer for a problem regardless of the instance. Its runtime is defined as the number of basic arithmetic operations conducted by the algorithm. For a polynomialtime algorithm, the runtime is polynomially bounded by the encoding size of the input.

A decision problem is a problem whose only possible answers are "yes" or "no". Complexity classes define sets of problems with the same runtime boundedness. The complexity class $\mathrm{P}$ contains all decision problems that can be answered by a polynomial-time algorithm. Furthermore, the complexity class NP contains the decision problems for which a certificate exists and a polynomial-time algorithm can verify a "yes"-answer given that certificate. This is less restrictive than the complexity class $\mathrm{P}$ and yields to $\mathrm{P} \subseteq \mathrm{NP}$.

Note that complexity classes are defined for decision problems. We need a reformulation to use these classifications for optimization problems. For this purpose, we ask for a feasible point restricted to certain objective value and use binary search to find the optimal value. This is harder than the decision problem. Luckily, binary search runs in at worst logarithmic time such that, e.g., a polynomial-time algorithm for the single decision 
problem is still a polynomial-time algorithm. To refer to a complexity class for an optimization problem, we talk about the complexity class of the corresponding decision problem.

A concept to classify a wider range of problems is NP-hardness. A problem is said to be NP-hard if every decision problem from NP can be polynomially reduced to it. This means that, with a polynomial-time algorithm for this NP-hard problem, we can solve every problem of NP in polynomial time. In other words, NP-hard problems are at least as hard as the hardest problems in NP.

An NP-complete problem belongs to the NP and the NP-hard complexity classes. As NP is defined for decision problems, NP-completeness can only be valid for decision problems as well. Cook [34] detected such an NP-complete problem, named Boolean satisfiability problem (SAT), for the first time. Garey and Johnson [59] and Karp [95] extended the list to a wide range of problems.

It is an open question whether $\mathrm{P}=\mathrm{NP}$; see Fortnow [56]. In particular, the Clay Mathematics Institute stated it as one of the eight millennium prize problems; see Carlson et al. [25]. To date, there is no polynomialtime algorithm for any NP-hard and, by implication, any NP-complete problem. This points to the hypothesis $\mathrm{P} \neq \mathrm{NP}$. If there would be such a polynomial-time algorithm, we could take it to solve every other problem of NP in polynomial time after reduction and conclude $\mathrm{P}=\mathrm{NP}$. Indeed, most people think that $\mathrm{P} \neq \mathrm{NP}$. This means, broadly spoken, that there are problems that are hard to solve and problems that are easy to solve.

Altogether, we use complexity classes to estimate the worst-case runtimes for solving certain problems. For optimization problems, we want to know if they are NP-hard or if their corresponding decision problems are in $\mathrm{P}$. The former implies exponential-time algorithms if $\mathrm{P} \neq \mathrm{NP}$. The latter confirms the existence of a polynomial-time algorithm.

\subsection{Linear Programming}

A linear program (LPs) in standard form is defined as

$$
\begin{array}{ll}
\min _{x} & c^{\top} x \\
\text { s.t. } & A x=b, \quad x \geq 0,
\end{array}
$$

with $c \in \mathbb{R}^{n}, b \in \mathbb{R}^{m}, A \in \mathbb{R}^{m \times n}$, and $x \in \mathbb{R}^{n}$. Over the years, an enormous amount of research has been done on LPs. For a detailed overview, we 
refer to the books of Chvátal [30] and Dantzig and Thapa [42, 43] and the references therein. Three algorithms, namely simplex algorithm, ellipsoid method, and interior-point algorithm, are central to solve LPs as (2.1).

The simplex algorithm was suggested by George Dantzig during the 1940s to solve LPs that occur in a military context; see Dantzig [39, 40]. The idea is to iterate over the extreme points of the polyhedron and check them for optimality. The simplex algorithm is highly efficient in practice; see Bixby [15]. Unfortunately, the worst-case complexity is exponential time; see Klee and Minty [98].

Leonid Khachiyan proves the polynomial-time solvability of LPs with the ellipsoid method; see Khachiyan [96, 97]. This shows that the corresponding decision problems of LPs lie in P. On the other hand, the ellipsoid method is not efficient in practice as it produces numerical problems. The idea is to overlap the polyhedron with an ellipsoid and check its center for feasibility. The method reduces the ellipsoid's size step by step to detect a feasible point or confirm an empty polyhedron. The ellipsoid method can be enhanced to an algorithm for solving LPs with binary search again.

A major contribution that incorporates high efficiency and polynomialtime solvability is the interior-point algorithm of Narendra Karmarkar; see Karmarkar [94]. It iterates over points inside of the polyhedron to approach the optimum.

These days, state-of-the-art solvers as Gurobi and Cplex mainly rely on the simplex and the interior-point algorithm; see Gurobi [80] and Cplex [36], respectively. The simplex algorithm is typically superior for small problems, whereas the interior-point algorithm plays its strengths for big LPs.

\subsection{Mixed-Integer Linear Programming}

A mixed-integer linear program (MIP) is defined as

$$
\begin{aligned}
\min _{x} & c^{\top} x \\
\text { s.t. } & A x \geq b, \quad x^{-} \leq x \leq x^{+}, \quad x_{\mathcal{C}} \in \mathbb{R}^{|\mathcal{C}|}, \quad x_{\mathcal{I}} \in \mathbb{Z}^{|\mathcal{I}|},
\end{aligned}
$$

with the sets $\mathcal{C}$ and $\mathcal{I}$ being the finite index sets of continuous and integer variables with $|\mathcal{C}|>0$ and $|\mathcal{I}|>0$, respectively. Moreover, $c \in \mathbb{R}^{n}, b \in \mathbb{R}^{m}$, $A \in \mathbb{R}^{m \times n}$, and $x \in \mathbb{R}^{n}$ with bounds $x^{-} \leq x \leq x^{+}$. MIPs are NP-hard, so we cannot expect polynomial-time algorithms unless $\mathrm{P}=\mathrm{NP}$. More particularly, even the corresponding decision problems of pure integer programming 
with only binary variables are NP-complete and, as a consequence, NP-hard; see Karp [95].

To solve MIPs as (2.2), Gomory [69-71] proposes LP relaxations with relaxed integrality and uses the simplex algorithm to solve them. Afterward, he inserts cutting planes to cut off the fractional solutions and solves an LP again to obtain an integral solution any time soon.

Another crucial idea is the branch-and-bound algorithm; see Dakin [38] and Land and Doig [104]. Branch-and-bound solves LP relaxations with relaxed integrality iteratively and fixes variables to integrality during the process step by step.

Branch-and-cut combines these two concepts in a natural and intuitive way. Crowder and Padberg [37] propose such an algorithm for the traveling salesman problem where cutting planes are added for all LPs during branchand-bound.

Another common method to solve MIPs is to decompose them. Benders [14] proposes to reformulate the original problem into a model with less variables and more constraints by projection. A master problem solves this reformulated model with a subset of constraints. Afterward, a subproblem checks this optimal solution for the master problem for feasibility of the original problem. If feasibility does not hold, the subproblem delivers cuts, so-called Benders cuts, to remove the proposed master problem's solution. As this procedure delivers new rows, we talk about row generation. Dantzig and Wolfe [44] develop a reformulation that removes constraints but increases the amount of variables instead. A master problem solves the reformulated model but only with a subset of variables. Afterward, a subproblem checks this feasible solution for the master problem for optimality of the original problem. If optimality does not hold, the subproblem delivers variables, i.e., columns, to improve the proposed, not optimal, master problem's solution. As this procedure delivers new columns, we talk about column generation. Dantzig and Wolfe [44] describe the method for LPs, but it can be enhanced for MIPs as well.

The main difference between these methods is that Benders [14] generates optimal solutions that are potentially infeasible, while Dantzig and Wolfe [44] generate feasible solutions that are potentially not optimal. For the case of LPs, duality theory covers the relation and is used for the proofs in Benders [14] and Dantzig and Wolfe [44].

With these basics at hand, considerable research attention is directed toward the best possible algorithmic configuration to solve MIPs. This attention involves different cutting planes, heuristics, warm starts for the 
repeated and similar LPs, decomposition methods, and many more. A huge amount of research is devoted to these questions. Broad and detailed descriptions are given in the books of Conforti et al. [33], Nemhauser and Wolsey [123], Schrijver [147], and Wolsey [160].

The state-of-the-art solvers such as Gurobi, Cplex, and Scip use many of these ideas to solve MIPs; see Gurobi [80], Cplex [36], and Maher et al. [110] respectively. Nowadays, these solvers are so fast, stable, robust, and reliable that using MIP solvers as a workhorse for many different kinds of problems is a rational choice that we can exploit for the developments and the numerical results in Chapter 4 and Chapter 5.

\subsection{Mixed-Integer Nonlinear Programming}

A mixed-integer nonlinear program (MINLP) is defined as

$$
\begin{array}{ll}
\min _{x} & h(x) \\
\text { s.t. } & A x \geq b, \quad x^{-} \leq x \leq x^{+}, \quad x_{\mathcal{C}} \in \mathbb{R}^{|\mathcal{C}|}, \quad x_{\mathcal{I}} \in \mathbb{Z}^{|\mathcal{I}|}, \\
& f(x) \leq 0,
\end{array}
$$

with the sets $\mathcal{C}$ and $\mathcal{I}$ being the finite index sets of continuous and integer variables with $|\mathcal{C}|>0$ and $|\mathcal{I}|>0$, respectively. Moreover, $c \in \mathbb{R}^{n}, b \in \mathbb{R}^{m}$, $A \in \mathbb{R}^{m \times n}$, and $x \in \mathbb{R}^{n}$ with bounds $x^{-} \leq x \leq x^{+}$. MINLPs form an important class of optimization problems because they combine the possibility of modeling nonlinear aspects and discrete decisions. Unfortunately, MINLPs are in general undecidable; see Jeroslow [92]. This can be fixed by ensuring that the feasible set is compact what we assume for the rest of this thesis and what we obtain due to the bounds in (2.3b). In this case, MIPs can be reduced to MINLPs and NP-hardness holds again.

Solving MINLPs as (2.3) is a highly active field of research; see Belotti et al. [13] for a recent survey. To classify MINLPs, we distinguish between convex and nonconvex. We assume that the objective function is linear as a nonlinear objective can always be transferred to the constraint matrix. Convex MINLPs stand for a convex feasible set neglecting integrality. Convex constraint functions in (2.3c) ensure convex MINLPs. As it is often the case in optimization, convexity renders MINLPs much easier to solve. On the other hand, nonconvex MINLPs stand for a nonconvex feasible set even neglecting integrality.

Both, convex and nonconvex MINLPs, are tackled by an alternating process of convexification and refinement in general. Convexification ensures 
a relaxation of the original problem, which is probably even linearized, whereas refinement ensures finer relaxations in the course of the algorithms.

Convex Mixed-Integer Nonlinear Programming Convex MINLPs are typically tackled by considering the MIP and the NLP part.

One way is the NLP-based branch-and-bound of Gupta and Ravindran [79] that uses the branch-and-bound procedure from MIP theory. The main difference is that the iteratively solved relaxations are convex NLPs instead of LPs now. Another way is a branch-and-cut method for 0-1 mixed convex programming; see Stubbs and Mehrotra [152].

A further approach is to decompose the MINLP into an alternating sequence of master problems and subproblems. Similar to Benders [14] for MIPs, the generalized Benders' decomposition in Geoffrion [68] reformulates the original problem by projection. The result is an MIP master problem with less variables and more constraints again. Based on Benders [14], Geoffrion [68] constructs an NLP subproblem and relies on nonlinear duality theory. He proves convergence by closing the gap between lower and upper bound. Duran and Grossmann [50], Fletcher and Leyffer [55], and Leyffer [107] reformulate the original problem by a so-called outer approximation. Hereby, the MIP master problem has the same variables, but the nonlinearities are replaced by linear constraints at linearization points. The subproblem delivers new linearization points and the procedure ensures the relaxation property of the master problem. Outer approximation has a successful version called LP/NLP-based branch-and-bound proposed by Quesada and Grossmann [132]. Hereby, the MIP master problems are not solved to the end, but feasible solutions inside of the branch-and-bound tree are used to produce new linearization points along the way. Note that generalized Benders' decomposition is a particular case of outer approximation; see Kocis and Grossmann [100]. The latter is the state-of-the-art approach for solving convex MINLPs.

A third way to solve convex MINLPs is the extended cutting plane method by Westerlund and Pettersson [159] that decomposes the problem as well. Compared to outer approximation, it abandons the subproblem and derives new linearization points from the master problem's solution directly. Although this implies weaker cuts, the extended cutting plane method works fine for problems with a moderate degree of nonlinearity. 
Nonconvex Mixed-Integer Nonlinear Programming Additionally to the already mentioned Belotti et al. [13], the survey Burer and Letchford [23] and the book Tawarmalani and Sahinidis [153] provide nice overviews about specific nonconvex mixed-integer nonlinear programming algorithms.

The state-of-the-art approach is to decompose the nonconvex set into convex feasible sets in a so-called spatial branch-and-bound algorithm; see Smith and Pantelides [149, 150]. Spatial branch-and-bound solves the problem over a convexified set yielding an MIP or a convex MINLP. If feasibility does not hold, it splits up the feasible set by branching. Afterward, the method convexifies the single problems again and performs a branchand-bound algorithm. The main questions with such an approach are how to convexify an arbitrary nonconvex set and how to implement the branching step to improve the refinement of the nonlinear functions.

McCormick [118] proposes the state-of-the-art approach to convexify a nonconvex set using factorization. Factorable nonlinear functions are restricted to bivariate addition, bivariate multiplication, and some basic univariate functions. Moreover, it is a typical assumption for general nonconvex MINLPs to contain factorable nonlinear functions. In this case, we can reformulate the nonlinear functions to several basic univariate functions. To convexify the univariate functions, extensive literature studies are available; see, e.g., Liberti and Pantelides [108], McCormick [118], Smith and Pantelides [150], and Tawarmalani and Sahinidis [153, 154].

In the MIP context, branching on fractional integer variables is natural. For MINLPs, branching on continuous variables might make sense as well; see Smith and Pantelides [149, 150] again. Furthermore, branching strategies gain from strong bound tightening techniques. Especially Ryoo and Sahinidis [134, 135] develop optimality-based and feasibility-based range reductions and establish a so-called branch-and-reduce algorithm. Further developments to improve refinement are presented in Belotti et al. [11], Belotti [12], and Tawarmalani and Sahinidis [154]. All procedures share the idea of refining the nonlinear functions inside of a branch-and-bound tree successively.

Another possibility to solve nonconvex MINLPs is to piecewise linearize the nonlinear functions after factorization again. Martin and Möller [115], Martin et al. [116], and Möller [120] investigate this approach and Geißler et al. [61-64] and Morsi [122] show how to maintain the relaxation property. After checking for feasibility again, possible refinement steps are investigated by Burlacu et al. [24] and Geißler [60]. The linearization 
methods itself are presented in Dantzig [41] and Markowitz and Manne [114]. We refrain from reviewing the large literature on piecewise linear approximation of nonlinear functions but refer the reader to the references in the hitherto cited papers.

A whole zoo of solvers for MINLPs, which mainly use the described procedures, exist and we mention the most relevant ones. Gurobi and Cplex restrict themselves to specific convex, that is quadratic, MINLPs; see Gurobi [80] and Cplex [36], respectively. Additionally, Bonmin solves general convex MINLPs, while Baron, Couenne, and Scip promise to solve arbitrary MINLPs; see Bonmin [18], Baron [9], Couenne [35], and Maher et al. [110], respectively. We have to mention that general-purpose global MINLP solvers do not deliver that fast and reliable solutions as one might hope so far. Nevertheless, these solvers can be used for comparable small problems what we exploit for the developments and the numerical results in Chapter 4. 


\section{Gas Transport Optimization}

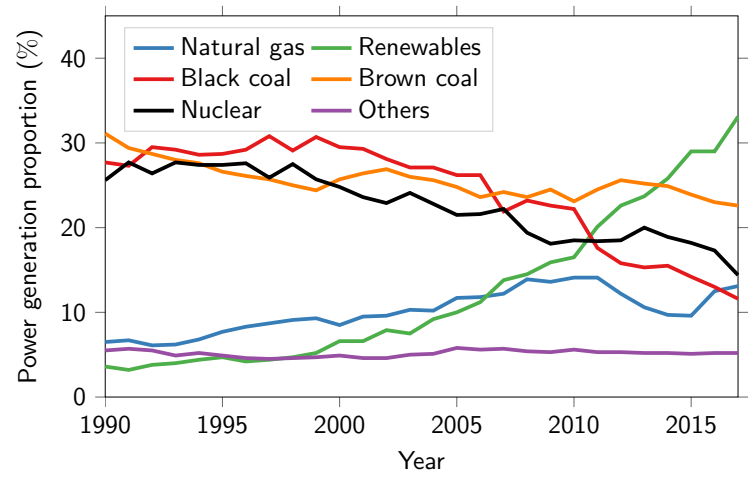

Figure 3.1: Gross power generation in Germany; see AG Energiebilanzen e.V. [1].

In this chapter, we provide basic information about natural gas and about our application of gas transport optimization. In Germany, our main focus from now on, a politically intended energy turnaround has stirred up the way of power generation in the last decade; see Figure 3.1. The proportion of natural gas rises slightly in that context and gives priority to questions of gas transport. In particular, Germany does not have relevant gas fields and mainly Russia, the Netherlands, and Norway deliver the commodity; see AG Energiebilanzen e.V. [1]. Consequently, gas transportation through pipelines and its mathematical optimization is a highly active field of research of applied optimization; see the detailed overviews Domschke et al. [49], Hante et al. [83], Koch et al. [99], and Ríos-Mercado and Borraz-Sánchez [133] and the references therein.

Section 3.1 gives an introduction and overview of the German energy industry with focus on natural gas and explains Figure 3.1 in detail. Section 3.2 presents the mathematical models of the relevant gas network elements. Finally, we provide a literature study of how gas transport problems are typically solved in Section 3.3. 


\subsection{The German Energy Industry}

Modern industrial societies such as Germany are causing a lot of trouble concerning the natural environment. These troubles are mainly caused by greenhouse gas emissions and nuclear power generation among others.

Greenhouse gas emissions are responsible for global warming; see Blunden and Arndt [16] and the references therein. Energy industries, e.g., power generation, manufacturing industries, construction, and transport are mainly responsible for the emissions; see Umweltbundesamt [156, Chapter 3.1]. Fossil energy sources such as black and brown coal were, in addition to nuclear material, the main commodities for the power generation during the '90s; see Figure 3.1. These fossil sources are cheap but produce high amounts of greenhouse gas when used for power generation; see Wagner et al. [158].

On the other hand, nuclear power generation has the problem of the final nuclear waste repository and is risky in the daily run; see Lelieveld et al. [105]. Nuclear power plants produce contaminated material and its repository remains an open question because nuclear radiation survives for thousands of years. Furthermore, the well-known Three Mile Island (03/28/1979), Chernobyl (04/26/1986) and Fukushima (03/11/2011) disasters in the USA, the former Soviet Union, and in Japan, respectively, pointed out the risk in operation.

All these facts have forced German politicians to action; see Jacobsson and Lauber [91]. Germany makes efforts to reduce greenhouse gas emissions by eliminating fossil fuels like coal. Moreover, a planned nuclear power phaseout by 2022 shall omit the risk and the long-term problems of nuclear energy; see Bundesgesetzblatt [21] for the law adopted in 2011. Renewable energies from wind, water, or sun shall replace the resulting energy gap. Various renewable energy laws have been passed during the '90s and '00s to support and subsidize these renewable energy technologies; see Bundesgesetzblatt [22] for the most important law during that process. This whole process is called the energy turnaround. It increases power generation from renewable energies and decreases it from fossil and nuclear energy; see Figure 3.1. Consequently, Germany reduced greenhouse gas emissions by $26.5 \%$ from 1990 to 2015; see Umweltbundesamt [156, Chapter 2.3]. We remark that additional developments, e.g., improved efficiency factors, play a role as well. Nevertheless, the reorganization of power generation had a significant effect; see Umweltbundesamt [156, Chapter 2.1].

How does natural gas come into play now? Before the energy turnaround, the base load of the electricity network was ensured by coal-fired and 
nuclear power plants and, at a lower percentage, by gas-fired power plants; see Figure 3.1 again. The supply was smooth and omitted great variations in the network. As opposed to this, renewable energies are often exposed to the weather whose fluctuations directly translate into the network. This is not desirable as fluctuations lead to unwanted instabilities. An important role to stabilize these fluctuations falls to gas-fired power plants.

Gas-fired power plants start up and shut down quickly. Therefore, it makes sense to combine them with renewable energy production to balance fluctuations. It is seen as an energy source for a transition period until renewable resources suffice to produce all power by themselves. Moreover, gas networks can serve as storages. The network stores gas after excess power is converted to natural gas in a so-called power-to-gas procedure. Finally, energy production with natural gas emits less greenhouse gas compared to coal; see Wagner et al. [158] again.

All these circumstances led to political support for gas-fired power plants. Nonetheless, the proportion of power generated by natural gas decreased from 2010 to 2014; see Figure 3.1 again. Extremely complicated regulatory rules in the German electricity market explain this development; see Asendorpf [5] for a simplified explanation in a popular scientific way. Natural gas was temporarily even displaced by much more environmentally harmful energy sources as brown coal. Fortunately, this market failure seems to alleviate.

Natural Gas plays an important role for the energy turnaround to succeed. This motivates the German authorities on behalf of the German Research Foundation ${ }^{1}$ to promote the academic research project "SFB Transregio 154: Mathematical Modeling, Simulation and Optimization using the Example of Gas Networks" "with Prof. Dr. Alexander Martin as a speaker from 2014 on. Various research groups from German universities - which are Friedrich-Alexander-Universität Erlangen-Nürnberg ${ }^{3}$, Humboldt-Universität zu Berlin ${ }^{4}$, Technische Universität Berlin ${ }^{5}$, Technische Universität Darmstadt $^{6}$, and Universität Duisburg-Essen ${ }^{7}$ - and research institutes - which are Weierstraß-Institut für Angewandte Analysis und Stochastik Berlin ${ }^{8}$

\footnotetext{
${ }^{1}$ http://www.dfg.de

${ }^{2}$ http://trr154.fau.de

${ }^{3}$ http://www.fau.de

${ }^{4}$ http://www.hu-berlin.de

${ }^{5}$ http://www.tu-berlin.de

${ }^{6}$ http://www.tu-darmstadt.de

${ }^{7}$ http://www.uni-due.de

${ }^{8}$ http://www.wias-berlin.de
} 
and Zuse Institute Berlin ${ }^{9}$ - as well as the biggest German gas transport company Open Grid Europe $\mathrm{GmbH}^{10}$ with place of business in Essen are involved. Such gas transport companies or operators of gas networks are exclusively responsible for the transportation and do not deal in the commodity; see Gotzes et al. [72] for the regulatory explanations. As part of the project, the author of this thesis focuses on a central area of such gas transport companies: gas transport optimization.

\subsection{Modeling Gas Networks}

Gas transport optimization requires a precise understanding of the physical principles of natural gas. In the following, we introduce the gas network elements together with their mathematical models. For additional details about gas modeling we refer to Domschke et al. [49], Koch et al. [99], Lurie [109], and Ríos-Mercado and Borraz-Sánchez [133].

Natural gas is typically transported over large distances through a pipeline network. The state variables, pressure and mass flow, are governed by highly nonlinear gas physics and gas mainly flows from higher to lower pressures. Consequently, in order to transport the commodity over large distances through pipeline systems, the gas pressure is increased by compression. This is realized by compressor machines. On the other hand, opposite elements that reduce the gas pressure, which are control valves, or even close a connection, which are valves, are given. All these elements can be controlled, which adds discrete aspects to the problem.

Our gas network is modeled by a directed Graph $G=(V, A)$. The set of nodes $V$ is divided into entry nodes $V_{+}$, exit nodes $V_{-}$, and inner nodes $V_{0}$. Thus, $V=V_{+} \cup V_{-} \cup V_{0}$. Entry nodes $V_{+}$supply gas, while exit nodes $V_{-}$discharge gas. Inner nodes $V_{0}$ do not supply or discharge any gas. We remark that we only need the set $V$ for the rest of Chapter 3 . Nevertheless, we recapture the sets $V_{+}, V_{-}$, and $V_{0}$ in Chapter 4 and Chapter 5. Arcs $a=(u, w) \in A$ belong to different network elements and connect nodes $u, w \in V$. We distinguish passive and active network elements. Passive elements are exclusively pipes $A_{\mathrm{pi}}$, while active elements consist of valves $A_{\mathrm{va}}$, control valves $A_{\mathrm{cv}}$, and compressor machines $A_{\mathrm{cm}}$.

We consider the time horizon $\left[0, \mathcal{T}^{\max }\right]$ and a time discretization with time points $0=: t_{0}<t_{1}<\ldots<t_{K}:=\mathcal{T}^{\text {max }}$ and a resulting set of time

\footnotetext{
9 http://www.zib.de

${ }^{10} \mathrm{http}: / /$ www.open-grid-europe.com
} 
points $\mathcal{T}:=\left\{t_{0}, t_{1}, \ldots, t_{K}\right\}$. Our important set of variables to observe are pressure $p$, mass flow $q$, mass flux $\chi$, and the required power of the compressor machines $P$.

We define pressure variables $p$ at all nodes and for all time points:

$$
p_{u}^{t} \in\left[p_{u}^{-}, p_{u}^{+}\right] \quad \text { for all } u \in V, t \in \mathcal{T} .
$$

Moreover, we have mass flow variables $q$ at all nodes and for all time points to model supplied gas, discharged gas, or neither of both. These situations are given by positive values, negative values, or zero, respectively. Furthermore, mass flow variables $q$ at the beginning and at the end of all arcs are defined for all time points again and we obtain

$$
\begin{aligned}
q_{u}^{t} \in\left[q_{u}^{-}, q_{u}^{+}\right] & \text {for all } u \in V, t \in \mathcal{T}, \\
q_{a, u}^{t}, q_{a, w}^{t} \in\left[q_{a}^{-}, q_{a}^{+}\right] & \text {for all } a=(u, w) \in A, t \in \mathcal{T} .
\end{aligned}
$$

Besides, valves, control valves, and compressor machines have constant flow for all $t \in \mathcal{T}$. Thus, for the sake of simplicity and in addition to $(3.2 \mathrm{~b})$, we define variables

$$
q_{a}^{t}=q_{a, u}^{t}=q_{a, w}^{t} \quad \text { for all } a=(u, w) \in A_{\mathrm{va}} \cup A_{\mathrm{cv}} \cup A_{\mathrm{cm}}, t \in \mathcal{T} .
$$

We remark that this does not hold for pipes $A_{\mathrm{pi}}$. Exclusively for pipes, we additionally define mass flux variables

$$
\chi_{a, u}^{t}, \chi_{a, w}^{t} \in\left[\chi_{a}^{-}, \chi_{a}^{+}\right] \quad \text { for all } a=(u, w) \in A_{\mathrm{pi}}, t \in \mathcal{T}
$$

at the beginning and at the end of all pipes and for all time points. Finally, we define variables describing the power for compression by

$$
P_{a}^{t} \in\left[P_{a}^{-}, P_{a}^{+}\right] \quad \text { for all } a \in A_{\mathrm{cm}}, t \in \mathcal{T} .
$$

\subsubsection{Nodes}

We model continuity of mass at all nodes. Additional notations we need are the sets of ingoing and outgoing arcs for all nodes:

$$
\begin{aligned}
\delta^{\text {in }}(u) & :=\{a \in A: \exists w \in V \text { with } a=(w, u)\} & \text { for all } u \in V, \\
\delta^{\text {out }}(u) & :=\{a \in A: \exists w \in V \text { with } a=(u, w)\} & \text { for all } u \in V .
\end{aligned}
$$

Thus, continuity of mass at a node $u$ is described by

$$
q_{u}^{t}+\sum_{a \in \delta^{\mathrm{in}}(u)} q_{a, u}^{t}=\sum_{a \in \delta^{\mathrm{out}}(u)} q_{a, u}^{t} \quad \text { for all } u \in V, t \in \mathcal{T} .
$$

We denote (3.6) by mass flow conservation. 


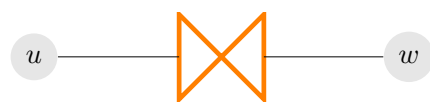

Figure 3.2: Technical symbol for a valve $a=(u, w) \in A_{\text {va }}$.

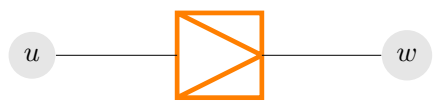

Figure 3.3: Technical symbol for a control valve $a=(u, w) \in A_{\mathrm{cv}}$.

\subsubsection{Arcs}

We give a description of our models for valves $A_{\mathrm{va}}$, control valves $A_{\mathrm{cv}}$, compressor machines $A_{\mathrm{cm}}$, and pipes $A_{\mathrm{pi}}$. Note that further elements as resistors or compressor groups are not described in this thesis as they are not incorporated into the test networks that we use in Chapter 4 and Chapter 5. The models for these elements can be found in Geißler et al. [62].

\section{Valves}

Valves $a=(u, w) \in A_{\mathrm{va}}$ are the most simple controllable elements in the considered gas transport networks. The technical symbol we use for valves can be seen in Figure 3.2. They can be in two different states: open or closed. With this notation at hand, we have

$$
\begin{aligned}
p_{u}^{t}=p_{w}^{t}, & \text { if valve } a=(u, w) \in A_{\mathrm{va}} \text { is open, } \\
q_{a}^{t}=0, & \text { if valve } a=(u, w) \in A_{\mathrm{va}} \text { is closed, }
\end{aligned}
$$

for all $t \in \mathcal{T}$. More detailed information about valves and a formulation as an MIP for a fixed $t$ can be found in Geißler et al. [62].

\section{Control Valves}

Slightly more complicated than valves are control valves $a=(u, w) \in A_{\mathrm{cv}}$. The technical symbol we use for control valves can be seen in Figure 3.3. Their state is distinguished into the active state, the bypass mode, and the closed state. If the control valve is active at time $t \in \mathcal{T}$, it can decrease the incoming gas pressure by a controllable amount

$$
\Delta_{a}^{t} \in\left[\Delta_{a}^{-}, \Delta_{a}^{+}\right] \quad \text { for all } a \in A_{\mathrm{cv}}, t \in \mathcal{T} .
$$




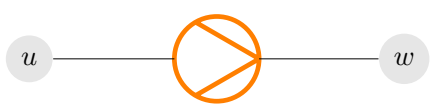

Figure 3.4: Technical symbol for a compressor machine $a=(u, w) \in A_{\mathrm{cm}}$.

The bypass mode and the closed state is the same as the open and the closed state for valves. In summary, we have

$$
\begin{aligned}
p_{u}^{t}-\Delta_{a}^{t}=p_{w}^{t}, & \text { if control valve } a=(u, w) \in A_{\mathrm{cv}} \text { is } \\
& \text { active, } \\
p_{u}^{t}=p_{w}^{t}, & \text { if control valve } a=(u, w) \in A_{\mathrm{cv}} \text { is in } \\
& \text { bypass mode, } \\
q_{a}^{t}=0, & \text { if control valve } a=(u, w) \in A_{\mathrm{cv}} \text { is } \\
& \text { closed, }
\end{aligned}
$$

for all $t \in \mathcal{T}$. We remark that control valves are unidirectional, i.e., they can reduce the pressure into one flow direction. For the opposite direction, they can only be closed or in bypass mode. This is why the technical symbol in Figure 3.3 states a direction, which is decreasing pressure from the left to the right. More detailed information about control valves and a specific formulation as an MIP for a fixed $t$ can again be found in Geißler et al. [62].

\section{Compressor Machines}

Compressor machines $a=(u, w) \in A_{\mathrm{cm}}$ are used to increase the inflow gas pressure to a higher outflow pressure. The technical symbol we use for compressor machines can be seen in Figure 3.4. As control valves, a compressor machine can be active, in bypass mode, or closed. If the compressor machine is in active status, it can increase the gas pressure by

$$
\Delta_{a}^{t} \in\left[\Delta_{a}^{-}, \Delta_{a}^{+}\right] \quad \text { for all } a \in A_{\mathrm{cm}}, t \in \mathcal{T} .
$$

The other states are the same as for control valves:

$$
\begin{aligned}
p_{u}^{t}+\Delta_{a}^{t}=p_{w}^{t}, & \text { if compressor machine } a=(u, w) \in A_{\mathrm{cm}} \text { is } \\
& \text { active, } \\
p_{u}^{t}=p_{w}^{t}, & \text { if compressor machine } a=(u, w) \in A_{\mathrm{cm}} \text { is in } \\
& \text { bypass mode, } \\
q_{a}^{t}=0, & \text { if compressor machine } a=(u, w) \in A_{\mathrm{cm}} \text { is } \\
& \text { closed, }
\end{aligned}
$$




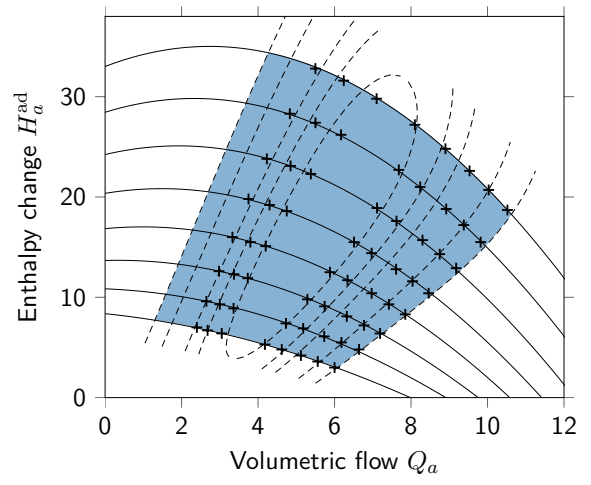

Figure 3.5: Characteristic diagram of a turbo compressor with operating range (blue).

for all $t \in \mathcal{T}$. Again, compressor machines are unidirectional, i.e., they can increase the pressure into one flow direction. For the opposite direction, they can be closed or in bypass. This is why the technical symbol in Figure 3.4 states a direction again, which is increasing pressure from the left to the right. The modeling of the exact feasible range of $\Delta_{a}^{t}$ is more complicated and depending on various influencing factors. We only consider turbo compressors, which are typically described by so-called characteristic diagrams shown in Figure 3.5. The volumetric flow rate

$$
Q_{a}^{t} \in\left[Q_{a}^{-}, Q_{a}^{+}\right] \quad \text { for all } a \in A_{\mathrm{cm}}, t \in \mathcal{T}
$$

is calculated by

$$
Q_{a}^{t}=\hat{R}_{\mathrm{s}} \hat{T} z_{u}^{t} \frac{q_{a}^{t}}{p_{u}^{t}} \quad \text { for all } a=(u, w) \in A_{\mathrm{cm}}, t \in \mathcal{T},
$$

and is given on the $x$-axis in Figure 3.5. The term $\hat{T}$ denotes the temperature. We remark that all our models are isothermal with constant temperature $\hat{T}$. This is a common assumption in gas transport optimization because gas coolers and heaters exist at compressor machines to control the outflow temperature. Note that all constants and parameters in the course of this thesis are tagged with a hat, e.g., $\hat{T}$. Additionally, $\hat{R}_{\mathrm{s}}$ denotes the specific gas constant and $z_{u}^{t}$ denotes the inflow compressibility factor. Many formulas for the calculation of the compressibility factor $z$ exist. We use the formula of the American Gas Association (AGA); see Králik et al. [101]. It is defined as

$$
z(p)=1+\hat{\alpha} p, \quad \hat{\alpha}=0.257 \frac{1}{\hat{p}_{\mathrm{c}}}-0.533 \frac{\hat{T}_{\mathrm{c}}}{\hat{p}_{\mathrm{c}} \hat{T}}
$$


with pseudocritical pressure $\hat{p}_{\mathrm{c}}$ and pseudocritical temperature $\hat{T}_{\mathrm{c}}$. Hence, the compressibility factor $z$ is a function $z=z(p) \in \mathbb{R}_{>0}$ depending on the pressure $p$. Furthermore, we assume the compressibility factor to be positive, which holds in gas transportation networks in general. With the formula of the American Gas Association at hand, we could use $z_{u}^{t}=1+\hat{\alpha} p_{u}^{t}$. Nevertheless, for the sake of simplicity, we define an average pressure parameter

$$
\hat{p}_{u}=\frac{p_{u}^{-}+p_{u}^{+}}{2} \quad \text { for all } a=(u, w) \in A_{\mathrm{cm}}
$$

and use it to define a constant compressibility factor

$$
\hat{z}_{u}=1+\hat{\alpha} \hat{p}_{u} \quad \text { for all } a=(u, w) \in A_{\mathrm{cm}}
$$

at the entry of a compressor machine. Finally, we write the volumetric flow rate as

$$
Q_{a}^{t}=\hat{R}_{\mathrm{s}} \hat{T} \hat{z}_{u} \frac{q_{a}^{t}}{p_{u}^{t}} \quad \text { for all } a=(u, w) \in A_{\mathrm{cm}}, t \in \mathcal{T} .
$$

Moreover, the specific change in adiabatic enthalpy

$$
H_{a}^{\mathrm{ad}, t} \in\left[H_{a}^{-}, H_{a}^{+}\right] \quad \text { for all } a \in A_{\mathrm{cm}}, t \in \mathcal{T}
$$

is calculated by

$$
\begin{array}{r}
H_{a}^{\mathrm{ad}, t}=\hat{R}_{\mathrm{s}} \hat{T} \hat{z}_{u} \frac{\hat{\kappa}}{\hat{\kappa}-1}\left[\left(\frac{p_{w}^{t}}{p_{u}^{t}}\right)^{(\hat{\kappa}-1) / \hat{\kappa}}-1\right] \\
\text { for all } a=(u, w) \in A_{\mathrm{cm}}, t \in \mathcal{T}
\end{array}
$$

and is given on the $y$-axis in Figure 3.5. We assume that the isentropic exponent $\hat{\kappa}=1.38$ is constant. Note that $p_{u}^{t}$ and $p_{w}^{t}$ denote the inflow and outflow pressure of the compressor unit, respectively. Additionally, we use the constant compressibility factor $\hat{z}_{u}$ again. In contrast to control valves, the realizable pressure change in compressors is not modeled independently of the specific flow through the unit; see the blue area in Figure 3.5 that depicts the feasible operating range of an exemplary machine. We have to model the nonlinear boundaries of this region what we do by using the nonlinear constraints

$$
f_{a}^{i}\left(Q_{a}^{t}, H_{a}^{\mathrm{ad}, t}\right) \leq 0, \quad i=1, \ldots, 4 \quad \text { for all } a \in A_{\mathrm{cm}}, t \in \mathcal{T} .
$$


Despite the discussed nonlinearity, tight linear relaxations for (3.15), (3.17), and (3.18) are available; see Geißler et al. [62]. Finally, the power for compression is derived from already defined variables by

$$
P_{a}^{t}=\frac{q_{a}^{t} H_{a}^{\text {ad }}}{\hat{\eta}_{a}^{\text {ad }}} \quad \text { for all } a \in A_{\mathrm{cm}}, t \in \mathcal{T}
$$

with $\hat{\eta}_{a}^{\text {ad }} \in[0,1]$ being the adiabatic efficiency modeling the deviation from the actual power. Again, linear relaxations are available. We use the MIP formulation for a fixed $t$ developed in Geißler et al. [62].

\section{Pipes}

We use pipes $A_{\mathrm{pi}}$ to transport gas through the network. Pipes are typically the most frequent elements in gas networks and we model them as onedimensional arcs $a=(u, w) \in A_{\mathrm{pi}}$. Gas flow is described by the Euler equations that are given as a system of nonlinear hyperbolic PDEs. These equations are derived from the more general Navier-Stokes equations and consist of the continuity equation, the balance of moments, and the energy equation; see Feistauer [54]:

$$
\begin{aligned}
\partial_{t} \rho+\partial_{x}(\rho v) & =0, \\
\partial_{t}(\rho v)+\partial_{x}\left(p+\rho v^{2}\right) & =-\frac{\hat{\lambda}_{a}}{2 \hat{D}_{a}} \rho v|v|-\hat{g} \rho \hat{s}_{a}, \\
\partial_{t}\left(\rho\left(\frac{1}{2} v^{2}+e\right)\right)+\partial_{x}\left(\rho v\left(\frac{1}{2} v^{2}+e\right)+p v\right) & =-\frac{\hat{k}_{a}}{\hat{D}_{a}}\left(T-T_{\iota}\right) .
\end{aligned}
$$

Here, $\rho=\rho(x, t) \in \mathbb{R}_{>0}$ denotes the density, $v=v(x, t) \in \mathbb{R}$ the velocity, $p=p(x, t) \in \mathbb{R}_{>0}$ the pressure, $e=e(x, t) \in \mathbb{R}_{>0}$ the internal energy, and $T=T(x, t) \in \mathbb{R}_{>0}$ the temperature of the gas. Furthermore, $T_{\iota}=T_{\iota}(x, t)$ denotes the temperature of the pipe's wall. Moreover, $x \in\left[0, \hat{L}_{a}\right]$ denotes the spatial coordinate of the pipe with length $\hat{L}_{a}$, while $t \in\left[0, \mathcal{T}^{\text {max }}\right]$ indicates the time point inside of the given time horizon. We remark that the functions depend on the pipe $a=(u, w) \in A_{\mathrm{pi}}$. Nevertheless, for the sake of simplicity, we refrain from indexing the functions. We further denote with $\hat{s}_{a}$ the slope, which we assume to be constant for every pipe, with $\hat{\lambda}_{a}$ the friction coefficient, with $\hat{D}_{a}$ the diameter, and with $\hat{k}_{a}$ the heat coefficient of the pipe. Finally, $\hat{g}$ denotes the gravitational constant.

Remark 3.2.1 (Variable $x$ vs. Spatial Coordinate $x$ ). We remark that the symbol $x$ is used in two different ways in this thesis. On the one hand, 
it denotes a general variable for our problem under consideration in (1.1). On the other hand, it represents the spatial coordinate for PDEs in (3.20). Both uses are standard conventions in their mathematical fields. This is the reason why we refrain from redefining any of them.

Many formulas to deduce the friction factor $\hat{\lambda}_{a}$ exist. We use the empirical formula of Prandtl-Colebrook; see Colebrook [32]. Remember that all our models are isothermal with constant temperature $T(x, t) \equiv \hat{T}$. This is a usual assumption in gas transport optimization as gas pipelines often lie under ground especially in Western Europe. Thus, the energy equation (3.20c) becomes obsolete and (3.20) reduces to

$$
\begin{aligned}
\partial_{t} \rho+\partial_{x}(\rho v) & =0 \\
\partial_{t}(\rho v)+\partial_{x}\left(p+\rho v^{2}\right) & =-\frac{\hat{\lambda}_{a}}{2 \hat{D}_{a}} \rho v|v|-\hat{g} \rho \hat{s}_{a} .
\end{aligned}
$$

Beyond the isothermal Euler equations (3.21), the equation of state is given. It connects the state quantities density $\rho$ and pressure $p$. It is defined as

$$
\rho \hat{R}_{\mathrm{s}} \hat{T} z=p
$$

Again, as for the compressor machine model, we use the formula of the American Gas Association (AGA) to calculate the compressibility factor $z$; see Králik et al. [101]. We repeat the definition:

$$
z(p)=1+\hat{\alpha} p, \quad \hat{\alpha}=0.257 \frac{1}{\hat{p}_{\mathrm{c}}}-0.533 \frac{\hat{T}_{\mathrm{c}}}{\hat{p}_{\mathrm{c}} \hat{T}}
$$

with pseudocritical pressure $\hat{p}_{\mathrm{c}}$ and pseudocritical temperature $\hat{T}_{\mathrm{c}}$. We still assume the compressibility factor to be positive. In contrast to the compressor machine model, we stick to the exact function (3.23) for the pipe model and do not simplify; see (3.13) and (3.14) to compare. Taken all together, we have the Euler equations (3.21), the equation of state (3.22), and the definition of the compressibility factor (3.23) in our isothermal model with the density function $\rho$, the velocity function $v$, and the pressure function $p$ being involved.

The next step is a transformation to get pressure functions and mass flux functions according to our initial definitions (3.1) and (3.4). In order to do so, we eliminate the density $\rho$ using the equation of state (3.22). Additionally, we can write

$$
\chi=\rho v
$$




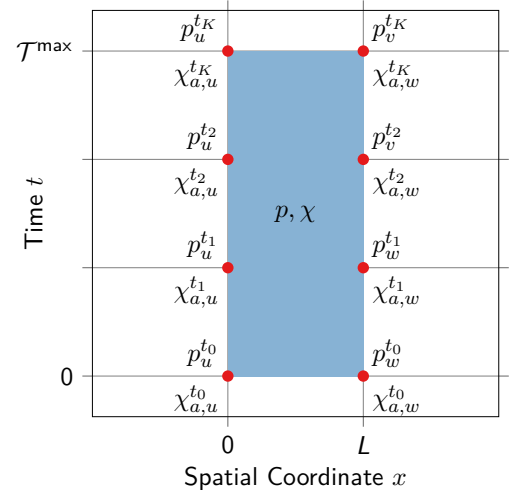

Figure 3.6: Domain of pressure and mass flux function (blue) according to (3.25) with variables (red) according to (3.1) and (3.4) on a pipe $a=(u, w)$.

with $\chi=\chi(x, t) \in \mathbb{R}$ denoting the mass flux. Again, $x \in\left[0, \hat{L}_{a}\right]$ denotes the spatial coordinate of the pipe with length $\hat{L}_{a}$, while $t \in\left[0, \mathcal{T}^{\text {max }}\right]$ indicates the time inside of the given time horizon. Eventually, we abbreviate $\hat{\theta}_{a}=\hat{\lambda}_{a} / \hat{D}_{a}$ and obtain

$$
\begin{aligned}
\partial_{t} p+\hat{R}_{\mathrm{s}} \hat{T} z(p) \partial_{x} \chi & =0, \\
\partial_{t} \chi+\partial_{x}\left(p+\hat{R}_{\mathrm{s}} \hat{T} z(p) \frac{\chi^{2}}{p}\right) & =-\frac{\hat{\theta}_{a} \hat{R}_{\mathrm{s}} \hat{T} z(p)}{2} \frac{\chi|\chi|}{p}-\frac{\hat{g} \hat{s}_{a}}{\hat{R}_{\mathrm{s}} \hat{T} z(p)} p,
\end{aligned}
$$

with pressure function $p$ and mass flux function $\chi$ depending on the spatial coordinate and the time. Moreover, the compressibility factor function $z$ depends on pressure $p$. All the rest consists of parameters.

After a spatial discretization at the beginning and at the end of the pipe combined with a time discretization, we obtain pressure variables and mass flux variables at the beginning and at the end of the pipe. The size of the spatial steps in the discretization of the PDEs is chosen equal to the length of the pipes. A finer discretization of each real pipe can be achieved by a representation with more than one pipe. We finally obtain the variables defined in (3.1) and (3.4). Figure 3.6 illustrates the discretization of (3.25).

We remark that we use mass flow variables and mass flux variables in parallel for pipes; see the definitions in (3.2b) and (3.4), respectively. The former are used for mass flow conservation (3.6) and the latter are used 
for the discretization of the pipe model (3.25). The values are linearly connected by

$$
\chi_{a, u}^{t}=\frac{q_{a, u}^{t}}{\hat{A}_{a}} \quad \text { and } \quad \chi_{a, w}^{t}=\frac{q_{a, w}^{t}}{\hat{A}_{a}} \quad \text { for all } a=(u, w) \in A_{\mathrm{pi}}, t \in \mathcal{T},
$$

with $\hat{A}_{a}$ being the cross-sectional area of the pipe.

\subsubsection{Summary}

We now obtain the entire model:

$$
\begin{aligned}
\text { pressure variables: } & (3.1) ; \\
\text { mass flow variables: } & (3.2),(3.3) \text {; } \\
\text { mass flux variables: } & (3.4) ; \\
\text { power variables: } & (3.5) ; \\
\text { mass flow/mass flux connection: } & (3.26) ; \\
\text { mass flow conservation: } & (3.6) ;
\end{aligned}
$$

valve, control valve, compressor model: linearized (3.7)-(3.19);

$$
\text { pipe model: (3.23), (3.25). }
$$

The model (3.27a) $-(3.27 \mathrm{~g})$ is given for all $t \in \mathcal{T}$ separately and dependencies across time do not exist. It is an MIP. This is obvious for (3.27a)-(3.27f). Furthermore, the MIP models $(3.27 \mathrm{~g})$ for the active elements are explained in Section 3.2.2 and we refer to Geißler et al. [62] once more. We do not touch this part for the rest of this thesis. Taken all together, we strongly rely on the well-investigated stationary MIP models of the active elements, i.e., valves, control valves, and compressor machines.

On the other hand, pipe model $(3.27 \mathrm{~h})$ is given by a system of hyperbolic PDEs that comprises dependencies across time. Consequently, we deal with a mixed-integer PDE constrained problem. We capture and process the pipe model in Chapter 4 and Chapter 5. The same holds for the objective functions and the exact optimization tasks that are not defined so far.

\subsection{Literature Survey: Solving Gas Transport Optimization Problems}

Most approaches deal with models similar or equal to the model we introduce in Section 3.2. Again, we mention the recent surveys in this field of research; 
see Domschke et al. [49], Hante et al. [83], Koch et al. [99], and Ríos-Mercado and Borraz-Sánchez [133] and the references therein.

One branch of literature mainly focuses on discrete aspects of the models that are needed for describing discrete controls of devices like valves, control valves, or compressor machines. An accurate model consists of these discrete controls together with the PDE pipe model. The (global) optimization over such a system in its full beauty is by far out of reach of today's algorithmic technology and theoretical knowledge. Thus, a lot of research relies on simplifying the PDE model. For the stationary case, all time-dependent aspects are neglected. In general, early approaches for solving these gas transport problems are often based on dynamic programming (DP); see Carter [26] for a detailed overview. Furthermore, (penalty) alternating direction methods are used to combine mixed-integer and continuous solution strategies in a hybrid approach in Geißler et al. [65-67]. By far the most common approaches in the literature are (piecewise) linearization techniques for obtaining MIP models that can be solved with state-of-the-art MIP solvers to global optimality; see, e.g., Burlacu et al. [24], Geißler [60], Geißler et al. [62-64], Martin and Möller [115], Martin et al. [116], Möller [120], and Morsi [122]. Besides that, we also use these (piecewise) linearization techniques to couple the gas network with an electricity system, where the latter is a unit commitment problem:

[148] M. Sirvent, N. Kanelakis, B. Geißler, and P. Biskas. "Linearized model for optimization of coupled electricity and natural gas systems." In: Journal of Modern Power Systems and Clean Energy 5.3 (May 2017), pp. 364-374. DOI: 10.1007/s40565-017-0275-2.

The content of the article is not part of this thesis. For the more complicated transient case, Domschke et al. [48], Geißler et al. [61], Mahlke et al. [111], and Moritz [121] use the (piecewise) linearization techniques from the stationary case. Additionally, Mahlke et al. [112] develop promising heuristics to solve the corresponding problems. Recently, Hahn et al. [81] construct a finite volume scheme for the PDE constraints and present heuristics to solve the resulting MINLP. Moreover, extended nonlinear models of mathematical programs with equilibrium constraints (MPEC) that allow for switching of discrete states within continuous models are investigated; see Baumrucker and Biegler [10], Schmidt [138, 139], and Schmidt et al. [143, 144].

Another set of approaches assumes that the discrete controls are externally given and focuses on the nonlinear aspects of the problem. However, 
even after fixing the discrete decisions and simplifying the PDE model, the combination of highly nonlinear gas dynamics in pipes and typically nonlinear as well as nonconvex models of technical entities like, e.g., compressor machines, from the engineering literature already leads to NLP models that are themselves hard to solve; see Schmidt et al. $[145,146]$ for the stationary case. For the transient case, computational studies of NLP solution techniques for fully discretized systems can be found in Ehrhardt and Steinbach [51, 52] and Steinbach [151]. Additionally, an optimal control approach for transient gas network flow without discrete aspects is presented in Zlotnik et al. [161]. Finally, theoretical studies of controllability, stabilization, or the existence of stationary states of gas flow are topics of Banda and Herty [7], Banda et al. [8], Brouwer et al. [19], Gugat [74], and Gugat et al. [75, 78]. 



\section{Decomposition Methods for Mixed-Integer Nonlinear Programs}

In this chapter, we provide three algorithms to solve MINLPs, which is an NP-hard problem. Our contribution is to consider the case where we restrict the knowledge about the nonlinearities to a variable extent. These restrictions may occur due to problems constrained by differential equations or models that build upon black-box simulation runs. Depending on the level of our knowledge, our contribution consists of three new tailored decomposition algorithms that build upon an MIP master problem to be defined. We clarify their limitations, prove their correctness and finite terminations, and present promising numerical results for stationary gas transport problems. Moreover, we show the connection to already existing algorithms and to state-of-the-art general-purpose MINLP solvers. Taken all together, we rely on our guidelines of decomposing our problem and using MIP solvers as a workhorse again.

Chapter 4 is structured as follows. We formulate our problem, a general MINLP, in Section 4.1. We restrict the knowledge about the nonlinear functions by defining assumptions in Section 4.2. Afterward, a description of the basic algorithmic framework is described in Section 4.3. With these basics at hand, we propose three new decomposition based solution algorithms and prove their correct and finite terminations in Sections 4.4-4.6. It follows a literature survey and a discussion about the exact connection to other algorithms in Section 4.7. We make further remarks in Section 4.8 and use the algorithms for stationary gas transport problems in Section 4.9 where we provide promising numerical results.

We finally state that significant parts of this chapter are published in an international journal or are submitted for publication; see our articles Gugat et al. [77] and Schmidt et al. [141]. The author of this thesis made significant contributions for conceiving, developing, and elaborating all the presented contents that follow. In addition, we explicitly state results 
that originate from co-authors and where the author of this thesis did not contribute.

\subsection{Problem Statement}

We first define our optimization problem under consideration in Section 4.1.1. Afterward, we define our reformulated optimization problem in Section 4.1.2 and show constructively that we can use the latter to solve the former.

\subsubsection{General Mixed-Integer Nonlinear Program}

For this chapter, we define our optimization problem under consideration, a general MINLP, by copying (2.3):

$$
\begin{array}{ll}
\min _{x} & h(x) \\
\text { s.t. } & A x \geq b, \quad x^{-} \leq x \leq x^{+}, \quad x_{\mathcal{C}} \in \mathbb{R}^{|\mathcal{C}|}, \quad x_{\mathcal{I}} \in \mathbb{Z}^{|\mathcal{I}|}, \\
& f(x) \leq 0 .
\end{array}
$$

We remark that this general MINLP is discussed in Section 2.4 and is an example for Problem (1.1). The abstract constraints $x_{\mathcal{C}} \in \mathcal{X}$ from (1.1c) are replaced by nonlinear constraint functions in (4.1c).

\subsubsection{Reformulated Mixed-Integer Nonlinear Program}

We reformulate the general MINLP (4.1). A common assumption is that nonlinear functions (4.1a) and (4.1c) are factorable; see McCormick [118], Smith and Pantelides [150], Tawarmalani and Sahinidis [154], and Section 2.4. If this is the case, we can reformulate the general MINLP (4.1) to obtain problem

$$
\begin{array}{ll}
\min _{x} & c^{\top} x \\
\text { s.t. } & A x \geq b, \quad x^{-} \leq x \leq x^{+}, \quad x_{\mathcal{C}} \in \mathbb{R}^{|\mathcal{C}|}, \quad x_{\mathcal{I}} \in \mathbb{Z}^{|\mathcal{I}|} \\
& x_{i_{2}}=f_{i}\left(x_{i_{1}}\right) \quad \text { for all } i \in[\sigma] .
\end{array}
$$

In more detail, $c^{\top} x$ is a linear objective function with $c \in \mathbb{R}^{n}$. Moreover, $x_{i}=\left(x_{i_{1}}, x_{i_{2}}\right)$ denotes a pair of variables for all variable index pairs $i=\left(i_{1}, i_{2}\right) \in[\sigma]$ with $i_{1}, i_{2} \in \mathcal{C}$ and a finite set $[\sigma]:=\{0, \ldots, \sigma\}$. A function $f_{i}: \mathbb{R} \rightarrow \mathbb{R}$ couples the variables $x_{i_{1}}$ and $x_{i_{2}}$, i.e., $x_{i_{2}}=f_{i}\left(x_{i_{1}}\right)$ holds for all $i \in[\sigma]$. Problem (4.2) is a nonconvex MINLP because of the 


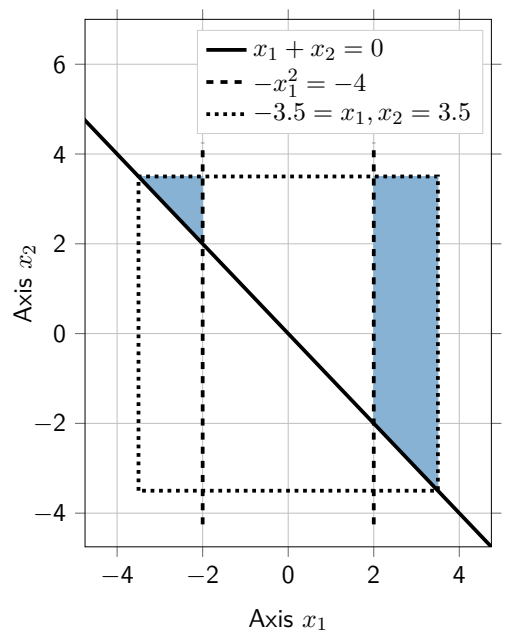

Figure 4.1: Nonconvex feasible set (blue) for Problem (4.3) neglecting integrality.

nonlinear equality constraints in (4.2c). We show the general reformulation procedure constructively with a minimal example.

Example 4.1.1. We define

$$
\begin{array}{ll}
\min _{x} & x_{1}^{2}+x_{2}^{2} \\
\text { s.t. } & x_{1}+x_{2} \geq 0, \quad-3.5 \leq x_{1}, x_{2} \leq 3.5, \\
& -x_{1}^{2} \leq-4, \\
& x_{1} \in \mathbb{Z}, \quad x_{2} \in \mathbb{R},
\end{array}
$$

as an example for the general nonconvex MINLP (4.1); see Figure 4.1 for an illustration of the nonconvex feasible set with neglected integrality. First, we shift the nonlinear objective to the constraints and insert a linear objective instead. We do this by defining a new variable $\eta_{1}$ :

$$
\begin{array}{ll}
\min _{x, \eta_{1}} & \eta_{1} \\
\text { s.t. } & x_{1}+x_{2} \geq 0, \quad-3.5 \leq x_{1}, x_{2} \leq 3.5 \\
& x_{1}^{2}+x_{2}^{2}-\eta_{1} \leq 0 \\
& -x_{1}^{2} \leq-4, \\
& x_{1} \in \mathbb{Z}, \quad x_{2}, \eta_{1} \in \mathbb{R} .
\end{array}
$$


Second, we separate all nonlinear functions by factorization. Thus, all nonlinear constraints are equality constraints with two variables involved:

$$
\begin{aligned}
\min _{x, \eta_{1}, \eta_{2}, \eta_{3}, \eta_{4}} & \eta_{1} \\
\text { s.t. } & x_{1}+x_{2} \geq 0, \quad-3.5 \leq x_{1}, x_{2} \leq 3.5, \\
& \eta_{2}+\eta_{3}-\eta_{1} \leq 0, \\
& \eta_{4} \leq-4, \\
& \eta_{2}=x_{1}^{2}, \\
& \eta_{3}=x_{2}^{2}, \\
& \eta_{4}=-x_{1}^{2}, \\
& x_{1} \in \mathbb{Z}, \quad x_{2}, \eta_{1}, \eta_{2}, \eta_{3}, \eta_{4} \in \mathbb{R} .
\end{aligned}
$$

Finally, if an integral variable $x_{i} \in \mathbb{Z}$ is involved in a nonlinearity, we replace it by a continuous variable and model equality with a linear constraint again:

$$
\begin{aligned}
\min _{x, \eta_{1}, \eta_{2}, \eta_{3}, \eta_{4}, \eta_{5}} & \eta_{1} \\
\text { s.t. } & x_{1}+x_{2} \geq 0, \quad-3.5 \leq x_{1}, x_{2} \leq 3.5, \\
& \eta_{2}+\eta_{3}-\eta_{1} \leq 0 \\
& \eta_{4} \leq-4 \\
& \eta_{5}=x_{1} \\
& \eta_{2}=\eta_{5}^{2} \\
& \eta_{3}=x_{2}^{2}, \\
& \eta_{4}=-\eta_{5}^{2}, \\
& x_{1} \in \mathbb{Z}, \quad x_{2}, \eta_{1}, \eta_{2}, \eta_{3}, \eta_{4}, \eta_{5} \in \mathbb{R} .
\end{aligned}
$$

This last formulation is in the form of Problem (4.2). We note that even if Problem (4.1) is a convex MINLP, the reformulated problem (4.2) is a nonconvex MINLP because of the nonlinear equality constraints in (4.2c) that yield a nonconvex feasible set.

In this chapter, we develop algorithms for the nonconvex MINLP (4.2), which is NP-hard. Taken the procedure of Example 4.1.1 into account, we can use these algorithms to solve the general MINLP (4.1). Additionally, and this is the interesting part, we restrict the knowledge about the nonlinearities by certain assumptions. 


\subsection{The Assumptions}

Our theoretical results depend on the assumptions made on the (in)exactness of function evaluations and on the knowledge of derivatives or Lipschitz constants for $(4.2 \mathrm{c})$. We note that these assumptions are restrictions that make the problems even harder as standard MINLPs. Depending on which assumption we use, our algorithmic approach differs. The easiest case is the following.

Assumption 4.2.1. We have an oracle that evaluates $f_{i}\left(x_{i_{1}}\right)$ and $f_{i}^{\prime}\left(x_{i_{1}}\right)$ for all $i \in[\sigma]$. Furthermore, all $f_{i}$ are strictly monotonic, strictly concave or convex, and differentiable with a bounded first derivative on $x_{i_{1}}^{-} \leq x_{i_{1}} \leq x_{i_{1}}^{+}$.

Note that we could drop the assumption of differentiability. In this case, $f_{i}$ might be differentiable almost everywhere for all $i \in[\sigma]$ and we can use sub- or supergradients at the points of non-differentiability.

Many mixed-integer optimization problems are constrained by nonlinear functions that do not possess desirable analytical properties like convexity. Thus, we define an alternative assumption.

Assumption 4.2.2. We have an oracle that evaluates $f_{i}\left(x_{i_{1}}\right)$ for all $i \in[\sigma]$. Furthermore, all $f_{i}$ are globally Lipschitz continuous on $x_{i_{1}}^{-} \leq x_{i_{1}} \leq x_{i_{1}}^{+}$with known global Lipschitz constant $L_{i}>0$.

Even more complicated is the setting where the functions $f_{i}$ cannot be evaluated exactly, but a reasonably good error bound is known.

Assumption 4.2.3. We have an oracle that provides an approximation $g_{i}\left(x_{i_{1}}\right)$ of $f_{i}\left(x_{i_{1}}\right)$ such that

$$
f_{i}\left(x_{i_{1}}\right) \in\left[g_{i}\left(x_{i_{1}}\right)-\varepsilon_{i}^{f}\left(x_{i_{1}}\right), g_{i}\left(x_{i_{1}}\right)+\varepsilon_{i}^{f}\left(x_{i_{1}}\right)\right]
$$

with known $\varepsilon_{i}^{f}\left(x_{i_{1}}\right)>0$ for all $i \in[\sigma]$. Furthermore, all $f_{i}$ are globally Lipschitz continuous on $x_{i_{1}}^{-} \leq x_{i_{1}} \leq x_{i_{1}}^{+}$with known global Lipschitz constant $L_{i}>0$. We assume that

$$
\varepsilon_{i}^{f}:=\sup _{x \in\left[x_{i_{1}}^{-}, x_{i_{1}}^{+}\right]} \varepsilon_{i}^{f}(x)
$$

is given a-priorily and that

$$
\varepsilon>2 \max _{i \in[\sigma]} \varepsilon_{i}^{f}
$$

holds. 


\subsection{Basic Algorithmic Framework}

Our idea is to decompose the problem into a master problem and a subproblem. These parts shall be tractable in practice, e.g., we want to obtain MIPs and small and tractable NLPs.

Hence, we decompose Problem (4.2) by splitting it up into a mixed-integer linear part and a nonlinear part. The master problem, an MIP, consists of everything apart from (4.2c), whereas the subproblems, various NLPs, comprise the handling of (4.2c).

The main algorithmic idea is that master problem and subproblem are solved alternately. The solutions of the subproblems are used to generate piecewise linear relaxations of the feasible set of (4.2c). The master problem uses these relaxations and iteratively gets better MIP relaxations of Problem (4.2) to solve. At the end, we obtain a globally optimal and approximately feasible solution of Problem (4.2) or a proof of infeasibility. The exact meaning of approximately feasible solution is explained at the end of Section 4.3.

We now introduce the basic ideas of our algorithms and some required notation in detail. We assume that the bounds $x^{-}$and $x^{+}$of Problem (4.2) give rise to a-priorily known compact boxes

$$
\Omega_{i}:=\left[x_{i}^{-}, x_{i}^{+}\right]:=\left[x_{i_{1}}^{-}, x_{i_{1}}^{+}\right] \times\left[x_{i_{2}}^{-}, x_{i_{2}}^{+}\right] \subset \mathbb{R}^{2}
$$

for all $i \in[\sigma]$ such that the graph of $f_{i}$ is contained in $\Omega_{i}$. If the graph is outside the box, we conclude infeasiblity.

The details of the algorithms vary depending on the assumptions we make in Section 4.2. Hence, we first provide a sketch of the generic algorithmic structure to be refined in the Sections 4.4-4.6. The algorithm constructs a sequence of subsets $\left(\Omega_{i}^{k}\right)_{k} \subseteq \Omega_{i}$ for all $i \in[\sigma]$ such that $\Omega_{i}^{k}$ converges to the graph of $f_{i}$ for iterations $k \rightarrow \infty$. For a better handling, we define $\operatorname{gr}\left(f_{i}\right)$ with

$$
\operatorname{gr}\left(f_{i}\right):=\left\{x_{i} \in \mathbb{R}^{2}: x_{i_{2}}=f_{i}\left(x_{i_{1}}\right)\right\} .
$$

For a precise construction of the relaxations $\Omega_{i}^{k}$ we need to specify what information on $f_{i}$ we use according to our assumptions in Section 4.2. Moreover, we assume that $\Omega_{i}^{k}$ are finite unions of polytopes, i.e.,

$$
\Omega_{i}^{k}:=\bigcup_{j \in\left[r_{i}^{k}-1\right]} \Omega_{i}^{k}(j)
$$

for all $i \in[\sigma]$ where $\Omega_{i}^{k}(j)$ are polytopes for all $j$ in some finite index set $\left[r_{i}^{k}-1\right]$. The precise definition of the sets $\Omega_{i}^{k}(j)$ follows later. Up to now 
it is only important that $\Omega_{i}^{k}$ is a union of polytopes that forms a relaxation of $\operatorname{gr}\left(f_{i}\right) \cap\left[x_{i}^{-}, x_{i}^{+}\right]$for all $i \in[\sigma]$.

The algorithm then alternately solves a master problem and subproblems. The master problem is defined over the relaxed sets $\Omega_{i}^{k}$ and the subproblems are used to effectively refine these relaxations to finally obtain an approximately feasible solution of Problem (4.2). With these preparations, we are now in the position to state the $k$ th master problem

$$
\begin{array}{lll}
\min _{x} & c^{\top} x \\
\text { s.t. } & A x \geq b, \quad x^{-} \leq x \leq x^{+}, \quad x_{\mathcal{C}} \in \mathbb{R}^{|\mathcal{C}|}, \quad x_{\mathcal{I}} \in \mathbb{Z}^{|\mathcal{I}|}, \\
& x_{i} \in \Omega_{i}^{k} \quad \text { for all } i \in[\sigma],
\end{array}
$$

that we solve to global optimality providing a solution $\widetilde{x}^{k}$. Note that the vector $x$ that is optimized in $(\mathrm{M}(k))$ is the same as the one used in Problem (4.2). However, when setting up a concrete MIP formulation for the constraints $x_{i} \in \Omega_{i}^{k}$, we have to extend the original variable vector by additional auxiliary variables that are required to formulate mixed-integer linear models of unions of polytopes.

If the master problem is infeasible, Problem (4.2) is infeasible because of the relaxation property. On the other hand, if the master problem's result is already an approximately feasible solution of Problem (4.2), we are done. If this is not the case, we need to improve our approximation. To this end, we consider the $k$ th subproblem providing a new linearization point on the graph of $f_{i}$. With this at hand, the subproblem of the $k$ th iteration reads

$$
\begin{aligned}
\psi^{2}\left(\widetilde{x}^{k}\right):=\min _{x} & \left\|x-\widetilde{x}^{k}\right\|_{2}^{2} \\
\text { s.t. } & x_{i} \in \operatorname{gr}\left(f_{i}\right) \quad \text { for all } i \in[\sigma] .
\end{aligned}
$$

Note that we choose the $\ell_{2}$ norm for the subproblem as it can directly be used in a continuous optimization problem. The Euclidean norm is squared to obtain a differentiable objective $\psi^{2}\left(\widetilde{x}^{k}\right)$. The solutions of $(\mathrm{S}(k))$ are denoted by $\stackrel{\circ}{x}^{k}$. By construction, the subproblem $(\mathrm{S}(k))$ has a nonempty feasible set. Moreover, we assume that the index pairs $i=\left(i_{1}, i_{2}\right)$ are non-overlapping, i.e., $i_{1} \neq \phi_{1}$ and $i_{1} \neq \phi_{2}$ for all $i \neq \phi$. This assumption can always be achieved by adding additional variables to Problem (4.2) and enforcing equality by the linear constraint system $A x \geq b$; see Example 4.1.1 for the general procedure. As a consequence, we show that the subproblem $(\mathrm{S}(k))$ can be split up such that single subproblems can be solved in parallel in every iteration $k$ of the algorithm. 
Lemma 4.3.1. The subproblems $(\mathrm{S}(k))$ are completely separable, i.e., we can solve the kth subproblem by solving the two-dimensional problem

$$
\begin{array}{rr}
\psi_{i}^{2}\left(\widetilde{x}_{i}^{k}\right):=\min _{x_{i}} & \left\|x_{i}-\widetilde{x}_{i}^{k}\right\|_{2}^{2} \\
\text { s.t. } & x_{i} \in \operatorname{gr}\left(f_{i}\right)
\end{array}
$$

for all $i \in[\sigma]$.

Proof. The constraint set

$$
x_{i} \in \operatorname{gr}\left(f_{i}\right) \quad \text { for all } i \in[\sigma]
$$

of subproblem $(\mathrm{S}(k))$ completely decouples along $i \in[\sigma]$. The same holds for the objective function

$$
\left\|x-\widetilde{x}^{k}\right\|_{2}^{2}=\sum_{i \in[\sigma]}\left\|x_{i}-\widetilde{x}_{i}^{k}\right\|_{2}^{2} .
$$

Consequently, the subproblem's solution $\left(\stackrel{\circ}{x}_{i}^{k}\right)_{i \in[\sigma]} \in \mathbb{R}^{2 \sigma}$ of $(\mathrm{S}(k))$ is constructed of the solutions $\stackrel{\circ}{x}_{i}^{k} \in \mathbb{R}^{2}$ of $(\mathrm{S}(i, k))$ for all $i \in[\sigma]$.

Finally, we concretize approximately feasible solutions in two different ways. We remark that the objective values $\psi_{i}^{2}\left(\widetilde{x}_{i}^{k}\right)$ of the subproblems $(\mathrm{S}(i, k))$ are a natural measure of feasibility for every $i \in[\sigma]$. Thus, we define $\ell_{2}-\varepsilon$-feasibility.

Definition 4.3.2 ( $\ell_{2}-\varepsilon$-feasibility). Let $\varepsilon>0$. A solution $\widetilde{x}^{k}$ of the master problem $(\mathrm{M}(k))$ is called $\ell_{2}-\varepsilon$-feasible if $\psi_{i}\left(\widetilde{x}_{i}^{k}\right) \leq \varepsilon$ for all $i \in[\sigma]$. Otherwise, it is called $\ell_{2}-\varepsilon$-infeasible.

Another concept for feasibility is given by comparing the true function values with the approximated function values. Therefore, we specify approximately feasible solutions by $\varepsilon$-feasibility.

Definition 4.3.3 ( $\varepsilon$-feasibility). Let $\varepsilon>0$. A solution $\widetilde{x}^{k}$ of the master problem $(\mathrm{M}(k))$ is called $\varepsilon$-feasible if $\left|f_{i}\left(\widetilde{x}_{i_{1}}\right)-\widetilde{x}_{i_{2}}\right| \leq \varepsilon$ for all $i \in[\sigma]$. Otherwise it is called $\varepsilon$-infeasible.

Definition 4.3.2 and Definition 4.3.3 are visualized in Figure 4.2a and Figure $4.2 \mathrm{~b}$, respectively. 


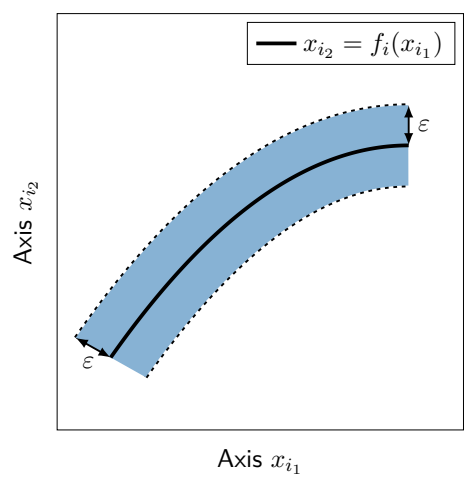

(a) $\ell_{2}-\varepsilon$-feasible set (blue) from Definition 4.3.2.

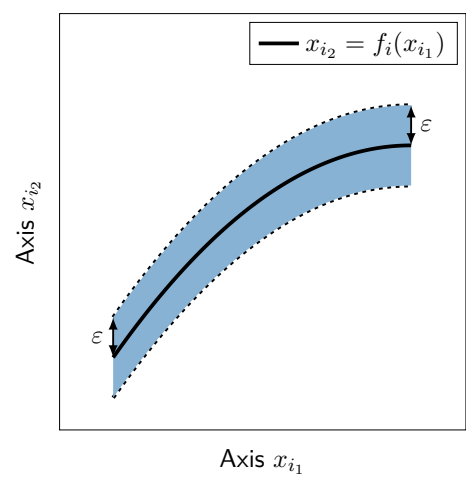

(b) $\varepsilon$-feasible set (blue) from Definition 4.3.3.

Figure 4.2: Illustration of Definition 4.3.2 and Definition 4.3.3.

\subsection{The Case of Strictly Monotonic and Strictly Concave or Convex Functions}

In this section, we assume that Assumption 4.2.1 holds. We present the details of the algorithm in regard to master problem $(\mathrm{M}(k))$ and subproblem $(\mathrm{S}(i, k))$ in Section 4.4.1. In Section 4.4.2, we then prove that the algorithm correctly terminates with a globally optimal $\ell_{2}-\varepsilon$-feasible point of Problem (4.2) or the indication of infeasibility. We remark that significant parts of this section can be found in our article Gugat et al. [77]. Note that the following descriptions amplify the short and concise presentations of the article.

\subsubsection{Derivation of the Algorithm}

Concerning Assumption 4.2.1, we remark that our results hold for all four combinations of strictly increasing or decreasing and strictly concave or convex functions. For the sake of simplicity, we fix the setting to strictly increasing and strictly concave for the rest of Section 4.4. The translation to the other cases is straight forward.

Master Problem The master problem $(\mathrm{M}(k))$ is a relaxation of Problem (4.2) and it is supposed to be an MIP. Instead of (4.2c), which is nonlinear, we take a piecewise linear relaxation of its feasible set $\operatorname{gr}\left(f_{i}\right) \cap\left[x_{i}^{-}, x_{i}^{+}\right]$ for all $i \in[\sigma]$ into account to specify $\Omega_{i}^{k}$ for iteration $k$. Beforehand, we 


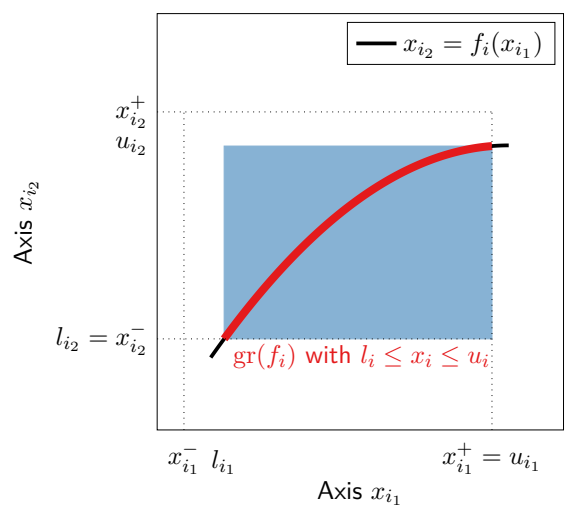

Figure 4.3: Bound tightening for $x_{i}$ with tightened box (blue).

strengthen the bounds of the involved variables using Assumption 4.2.1. The original bounds $x_{i}^{-} \leq x_{i} \leq x_{i}^{+}$are updated by $l_{i} \leq x_{i} \leq u_{i}$ with

$$
\begin{array}{ll}
l_{i_{1}}:=\max \left\{x_{i_{1}}^{-}, f_{i}^{-1}\left(x_{i_{2}}^{-}\right)\right\}, & u_{i_{1}}:=\min \left\{x_{i_{1}}^{+}, f_{i}^{-1}\left(x_{i_{2}}^{+}\right)\right\}, \\
l_{i_{2}}:=\max \left\{x_{i_{2}}^{-}, f_{i}\left(x_{i_{1}}^{-}\right)\right\}, & u_{i_{2}}:=\min \left\{x_{i_{2}}^{+}, f_{i}\left(x_{i_{1}}^{+}\right)\right\},
\end{array}
$$

for all $i \in[\sigma]$. See Figure 4.3 for an illustration.

For the piecewise linear relaxation of $\operatorname{gr}\left(f_{i}\right) \cap\left[l_{i}, u_{i}\right]$, we use a combination of the incremental method by Markowitz and Manne [114] and classical outer approximation by Duran and Grossmann [50] and Fletcher and Leyffer [55]. To construct the set $\Omega_{i}^{k}$ within the $k$ th master problem $(\mathrm{M}(k))$, we assume that there are values $x_{i_{1}}^{k, j} \in \mathbb{R}$ for all $j \in\left[r_{i}^{k}\right]$ with

$$
l_{i_{1}}=: x_{i_{1}}^{k, 0}<x_{i_{1}}^{k, 1}<\cdots<x_{i_{1}}^{k, r_{i}^{k}}:=u_{i_{1}}
$$

for all $i \in[\sigma]$. Now, as Assumption 4.2.1 gives an oracle for $f_{i}\left(x_{i_{1}}\right)$ and $f_{i}^{\prime}\left(x_{i_{1}}\right)$, there are values $f_{i}\left(x_{i_{1}}^{k, j}\right)=: x_{i_{2}}^{k, j} \in \mathbb{R}$ with

$$
l_{i_{2}}=: x_{i_{2}}^{k, 0}<x_{i_{2}}^{k, 1}<\cdots<x_{i_{2}}^{k, r_{i}^{k}}:=u_{i_{2}}
$$

and $f_{i}^{\prime}\left(x_{i_{1}}^{k, j}\right) \in \mathbb{R}$ with

$$
f_{i}^{\prime}\left(x_{i_{1}}^{k, 0}\right)>f_{i}^{\prime}\left(x_{i_{1}}^{k, 1}\right)>\cdots>f_{i}^{\prime}\left(x_{i_{1}}^{k, r_{i}^{k}}\right)
$$


for all $j \in\left[r_{i}^{k}\right]$ and for all $i \in[\sigma]$. The inequalities hold because of the strictly increasing and strictly concave functions. We abbreviate

$$
\begin{aligned}
\mathcal{L}_{i}^{k} & :=\left\{x_{i_{1}}^{k, 0}, x_{i_{1}}^{k, 1}, \ldots, x_{i_{1}}^{k, r_{i}^{k}-1}, x_{i_{1}}^{k, r_{i}^{k}}\right\}, \\
\mathcal{C}_{i}^{k} & :=\left\{x_{i_{2}}^{k, 0}, x_{i_{2}}^{k, 1}, \ldots, x_{i_{2}}^{k, r_{i}^{k}-1}, x_{i_{2}}^{k, r_{i}^{k}}\right\}, \\
\mathcal{G}_{i}^{k} & :=\left\{f_{i}^{\prime}\left(x_{i_{1}}^{k, 0}\right), f_{i}^{\prime}\left(x_{i_{1}}^{k, 1}\right), \ldots, f_{i}^{\prime}\left(x_{i_{1}}^{k, r_{i}^{k}-1}\right), f_{i}^{\prime}\left(x_{i_{1}}^{k, r_{i}^{k}}\right)\right\} .
\end{aligned}
$$

We now define the sets $\Omega_{i}^{k}$ for the $k$ th master problem $(\mathrm{M}(k))$ that are uniquely defined by the sets $\mathcal{L}_{i}^{k}, \mathcal{C}_{i}^{k}$, and $\mathcal{G}_{i}^{k}$. This piecewise linear relaxation $\Omega_{i}^{k}$ of $\operatorname{gr}\left(f_{i}\right) \cap\left[l_{i}, u_{i}\right]$ is modeled as an MIP:

$$
\begin{aligned}
& \Omega_{i}^{k}:=\left\{\left(x_{i_{1}}, x_{i_{2}}\right) \in \mathbb{R}^{2}: x^{-} \leq x \leq x^{+},\right. \\
& x_{i_{2}} \leq x_{i_{2}}^{k, j}+f_{i}^{\prime}\left(x_{i_{1}}^{k, j}\right)\left(x_{i_{1}}-x_{i_{1}}^{k, j}\right) \quad \text { for all } j \in\left[r_{i}^{k}\right], \\
& x_{i_{1}}=x_{i_{1}}^{k, 0}+\sum_{j=1}^{r_{i}^{k}}\left(x_{i_{1}}^{k, j}-x_{i_{1}}^{k, j-1}\right) \delta_{i}^{k, j}, \\
& x_{i_{2}} \geq x_{i_{2}}^{k, 0}+\sum_{j=1}^{r_{i}^{k}}\left(x_{i_{2}}^{k, j}-x_{i_{2}}^{k, j-1}\right) \delta_{i}^{k, j}, \\
& \delta_{i}^{k, j} \geq \omega_{i}^{k, j} \geq \delta_{i}^{k, j+1} \quad \text { for all } j \in\left[r_{i}^{k}-1\right] \backslash\{0\}, \\
& \delta_{i}^{k, j} \in[0,1] \quad \text { for all } j \in\left[r_{i}^{k}\right] \backslash\{0\}, \\
& \omega_{i}^{k, j}\left.\in\{0,1\} \quad \text { for all } j \in\left[r_{i}^{k}-1\right] \backslash\{0\}\right\} .
\end{aligned}
$$

The set $\Omega_{i}^{k}$ is depicted in Figure 4.4b. The linear overestimators (4.7b) are valid outer approximation cuts. The constraints (4.7c)-(4.7e) are piecewise linear underestimators by Assumption 4.2.1. The relaxed version of the incremental method uses the auxiliary variables in (4.7f) and (4.7g). We note that every single $\Omega_{i}^{k}(j)$ for all $j \in\left[r_{i}^{k}-1\right]$ from (4.6) is a triangle that is implicitly modeled by (4.7); see Figure $4.4 \mathrm{~b}$ again. By construction, we have the following propositions. 


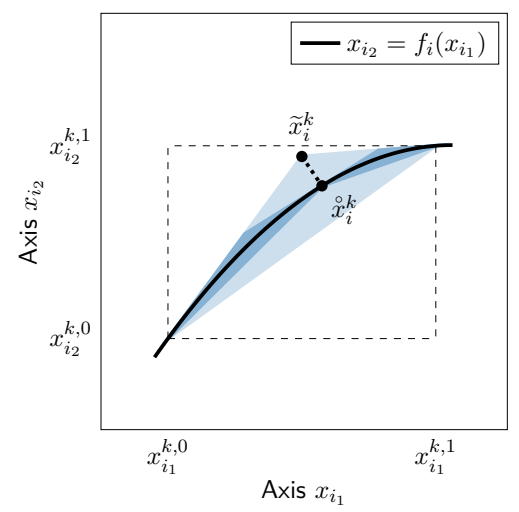

(a) Subproblem $(\mathrm{S}(i, k))$ for an $i \in[\sigma]$.

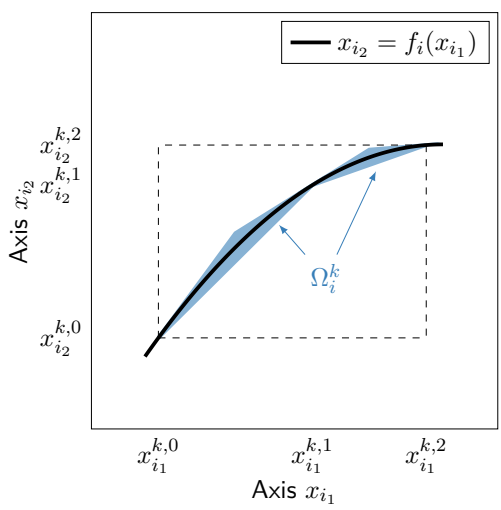

(b) Set $\Omega_{i}^{k}$ of master problem $(\mathrm{M}(k))$ for an $i \in[\sigma]$.

Figure 4.4: Setting for subproblem and master problem for Algorithm 4.1.

Proposition 4.4.1. For iteration $k$, we define $\Omega_{i}^{k}$ with $(4.7)$ for all $i \in[\sigma]$. Then

$$
\Omega_{i}^{k} \supseteq \operatorname{gr}\left(f_{i}\right) \cap\left[l_{i}, u_{i}\right]
$$

holds. Thus, the kth master problem $(\mathrm{M}(k))$ is a relaxation of $(4.2)$.

Proposition 4.4.2. For iteration $k$, we define $\Omega_{i}^{k}$ with $(4.7)$ for all $i \in[\sigma]$. Thus, the master problem $(\mathrm{M}(k))$ is an $\mathrm{MIP}$.

We note that an MIP can be solved in finite time, i.e., a standard MIP solver can compute a global optimal solution for $(\mathrm{M}(k))$ or prove infeasibility in finite time. Due to Proposition 4.4.1, we know that either $(\mathrm{M}(k))$ is solved to global optimality in finite time or Problem (4.2) is infeasible.

Subproblem Given a solution $\widetilde{x}_{i}^{k}$ of $(\mathrm{M}(k))$, the subproblems $(\mathrm{S}(i, k))$ are solved to either determine that the solution found by $(\mathrm{M}(k))$ is close enough to the original feasible set, or alternatively, to provide a new point $\stackrel{\circ}{x}_{i}^{k}$ with its derivative to be added to $\mathcal{L}_{i}^{k+1}, \mathcal{C}_{i}^{k+1}$, and $\mathcal{G}_{i}^{k+1}$, respectively. A subproblem $(\mathrm{S}(i, k))$ that determines the closest points on $\operatorname{gr}\left(f_{i}\right)$ with respect to the given master problems' solutions $\widetilde{x}_{i}^{k}$ is illustrated in Figure 4.4a. Note that Figure $4.4 \mathrm{~b}$ shows the master problem with the result of the subproblem in Figure 4.4a. The subproblems have a nonempty feasible set by construction. Moreover, the objective values of the subproblems are a natural measure for feasibility; see Definition 4.3.2. 
Algorithm 4.1 Decomposition Method in Case of Assumption 4.2.1

Input: Problem (4.2) and $\varepsilon>0$.

Output: If there is an $\ell_{2}-\varepsilon$-feasible point for Problem (4.2), the algorithm returns a globally optimal $\ell_{2}-\varepsilon$-feasible solution $\widetilde{x}$. Otherwise, it returns an indication of infeasibility.

1: Set $k \leftarrow 1$ and define $\mathcal{L}_{i}^{0}:=\left\{l_{i_{1}}, u_{i_{1}}\right\}, \mathcal{C}_{i}^{0}:=\left\{l_{i_{2}}, u_{i_{2}}\right\}$, and $\mathcal{G}_{i}^{0}:=\left\{f_{i}^{\prime}\left(l_{i_{1}}\right), f_{i}^{\prime}\left(u_{i_{1}}\right)\right\}$ for all $i \in[\sigma]$.

2: while true do

3: $\quad$ Solve master problem $(\mathrm{M}(k))$ with $\mathcal{L}_{i}^{k}, \mathcal{C}_{i}^{k}$, and $\mathcal{G}_{i}^{k}$ to global optimality where $\Omega_{i}^{k}$ is defined by (4.7) for all $i \in[\sigma]$.

4: $\quad$ if master problem $(\mathrm{M}(k))$ is infeasible then

5: return "Problem (4.2) is infeasible".

6: end if

7: $\quad$ Denote the solution of master problem $(\mathrm{M}(k))$ by $\widetilde{x}^{k}$.

8: $\quad$ Solve subproblems $(\mathrm{S}(i, k))$ for all $i \in[\sigma]$ yielding solutions $\stackrel{\circ}{x}_{i}^{k}$ and objective values $\psi_{i}^{2}\left(\widetilde{x}_{i}^{k}\right)$.

9: $\quad$ if $\psi_{i}\left(\widetilde{x}_{i}^{k}\right) \leq \varepsilon$ for all $i \in[\sigma]$ then

10: $\quad$ return globally optimal $\ell_{2}-\varepsilon$-feasible solution $\widetilde{x}^{k}$.

11: else

12: $\quad$ Set $\mathcal{L}_{i}^{k+1} \leftarrow \mathcal{L}_{i}^{k} \cup\left\{\stackrel{\circ}{x}_{i_{1}}^{k}\right\}, \mathcal{C}_{i}^{k+1} \leftarrow \mathcal{C}_{i}^{k} \cup\left\{\stackrel{\circ}{x}_{i_{2}}^{k}\right\}, \quad$ and $\mathcal{G}_{i}^{k+1} \leftarrow \mathcal{G}_{i}^{k} \cup\left\{f_{i}^{\prime}\left(\stackrel{\circ}{x}_{i_{1}}^{k}\right)\right\}$ for all $i \in[\sigma]$ with $\psi_{i}\left(\widetilde{x}_{i}^{k}\right)>\varepsilon$.

13: end if

14: $\quad$ Increase $k \leftarrow k+1$.

15: end while

Decomposition Algorithm We are now able to formally state the decomposition algorithm for solving Problem (4.2) in case of Assumption 4.2.1; see Algorithm 4.1. After the master problem is solved in Line 3, the algorithm checks whether we possess an $\ell_{2}-\varepsilon$-feasible point in Line 9 . If this is not the case, there are subproblems with $\ell_{2}-\varepsilon$-feasibility violation larger than $\varepsilon$. For these indices $i$, we then refine the relaxation by adding the corresponding subproblem's solution $\stackrel{\circ}{x}_{i_{1}}^{k}, \stackrel{\circ}{x}_{i_{2}}^{k}$, and the derivative $f^{\prime}\left(\stackrel{ }{x}_{i_{1}}^{k}\right)$ to the sets $\mathcal{L}_{i}^{k+1}, \mathcal{C}_{i}^{k+1}$, and $\mathcal{G}_{i}^{k+1}$, respectively in Line 12 . The algorithm returns a globally optimal $\ell_{2}$ - $\varepsilon$-feasible point of Problem (4.2) or the indication of infeasibility. 


\subsubsection{Convergence Results}

We now prove that Algorithm 4.1 is correct in the sense that it terminates with a globally optimal $\ell_{2}$ - $\varepsilon$-feasible point of Problem (4.2) or with the indication of infeasibility. The proof consists of two main statements. We first show that an $\ell_{2}-\varepsilon$-infeasible solution $\widetilde{x}^{k}$ of the $k$ th master problem is cut off in the next iteration $k+1$; see Theorem 4.4.6. This is due to suitable extensions of the sets $\mathcal{L}_{i}^{k}, \mathcal{C}_{i}^{k}$, and $\mathcal{G}_{i}^{k}$, which are augmented for all $i \in[\sigma]$ with $\psi_{i}\left(\widetilde{x}_{i}^{k}\right)>\varepsilon$. Note that the solution of the subproblem can also be seen as a separation oracle that yields new cutting planes for the last infeasible master problem solution. The correctness is then finally shown in Theorem 4.4.7. For the sake of simplicity and clarity, we exclude iteration indices $k$ in Section 4.4.2.

We first prove an auxiliary result in Lemma 4.4.4. Beforehand, we define auxiliary sets $\bar{R}$ and $\underline{R}$ where we need the notations hyp $\left(f_{i}\right)$ and epi $\left(f_{i}\right)$ for the hypo- and epigraph of the function as well as the notations hyp $\left(f_{i}\right)$ and $\operatorname{epi}_{\mathrm{S}}\left(f_{i}\right)$ for their strict versions.

Definition 4.4.3. Let $\widetilde{x}_{i} \in \Omega_{i}$ be part of a solution of the master problem $(\mathrm{M}(k))$ for $i \in[\sigma]$. For $\widetilde{x}_{i} \in \operatorname{hyp}\left(f_{i}\right)$, we define

$$
\underline{R}\left(\widetilde{x}_{i}\right):=\left\{x_{i} \in \operatorname{gr}\left(f_{i}\right): x_{i_{1}} \leq \widetilde{x}_{i_{1}}, x_{i_{2}} \geq \widetilde{x}_{i_{2}}\right\}
$$

and for $\widetilde{x}_{i} \in \operatorname{epi}\left(f_{i}\right)$, we define

$$
\bar{R}\left(\widetilde{x}_{i}\right):=\left\{x_{i} \in \operatorname{gr}\left(f_{i}\right): x_{i_{1}} \geq \widetilde{x}_{i_{1}}, x_{i_{2}} \leq \widetilde{x}_{i_{2}}\right\} .
$$

See Figure $4.5 a$ for an illustration of $\underline{R}\left(\widetilde{x}_{i}\right)$ and Figure $4.5 b$ for $\bar{R}\left(\widetilde{x}_{i}\right)$, respectively.

Lemma 4.4.4. Let $\widetilde{x}_{i} \in \Omega_{i}$ be part of a solution of the master problem $(\mathrm{M}(k))$ and let $\stackrel{\circ}{x}_{i}$ be the solution of the subsequent subproblem $(\mathrm{S}(i, k))$ for $i \in[\sigma]$. Then the following holds:

(a) If there exists an index $j \in\left[r_{i}\right]$ with $\left(x_{i_{1}}^{j}, x_{i_{2}}^{j}\right)=\left(\widetilde{x}_{i_{1}}, \widetilde{x}_{i_{2}}\right)$, then $\stackrel{\circ}{x}_{i}=\widetilde{x}_{i}$ and $\psi_{i}\left(\widetilde{x}_{i}\right)=0$.

(b) Suppose that $\psi_{i}\left(\widetilde{x}_{i}\right)>\varepsilon>0$ holds. Then there exists an in$\operatorname{dex} j \in\left[r_{i}-1\right]$ with

$$
\begin{array}{ll}
x_{i_{1}}^{j}<\widetilde{x}_{i_{1}}<x_{i_{1}}^{j+1}, & x_{i_{2}}^{j}<\widetilde{x}_{i_{2}}<x_{i_{2}}^{j+1}, \\
x_{i_{1}}^{j}<\stackrel{\circ}{x}_{i_{1}}<x_{i_{1}}^{j+1}, & x_{i_{2}}^{j}<\stackrel{\circ}{x}_{i_{2}}<x_{i_{2}}^{j+1} .
\end{array}
$$

Moreover, $\stackrel{\circ}{x}_{i} \in R\left(\widetilde{x}_{i}\right)$ if $\widetilde{x}_{i} \in \operatorname{hyp}{ }_{\mathrm{S}}\left(f_{i}\right)$ and $\stackrel{\circ}{x}_{i} \in \bar{R}\left(\widetilde{x}_{i}\right)$ if $\widetilde{x}_{i} \in \operatorname{epi}\left(f_{i}\right)$. 
Proof. (a) As $\widetilde{x}_{i} \in \Omega_{i}$ holds, it also fulfills the constraints (4.7). As the linearization points $\left(x_{i_{1}}^{j}, x_{i_{2}}^{j}\right)$ are chosen such that $x_{i_{2}}^{j}=f_{i}\left(x_{i_{1}}^{j}\right)$ holds for all $j \in\left[r_{i}\right]$, we have $f\left(\widetilde{x}_{i_{1}}\right)=f\left(x_{i_{1}}^{j}\right)=x_{i_{2}}^{j}=\widetilde{x}_{i_{2}}$. Consequently, $\widetilde{x}_{i}$ is $\ell_{2}$ - $\varepsilon$-feasible and the subproblem $(\mathrm{S}(i, k))$ yields the optimum $\stackrel{\circ}{x}_{i}=\widetilde{x}_{i}$ with optimal value $\psi_{i}\left(\widetilde{x}_{i}\right)=0$.

(b) Because of $\psi_{i}\left(\widetilde{x}_{i}\right)>\varepsilon>0$ and (a), there exists an index $j \in\left[r_{i}-1\right]$ with

$$
x_{i_{1}}^{j}<\widetilde{x}_{i_{1}}<x_{i_{1}}^{j+1} .
$$

Together with $(4.7 \mathrm{c})$ this implies $\delta_{i}^{1}=\cdots=\delta_{i}^{j}=1, \delta_{i}^{j+1} \in(0,1)$, and $\delta_{i}^{j+2}=\cdots=\delta_{i}^{r_{i}}=0$. Thus, $(4.7 \mathrm{~d})$ and monotonicity of $f_{i}$ according to Assumption 4.2.1 yield

$$
x_{i_{2}}^{j}<\widetilde{x}_{i_{2}}<x_{i_{2}}^{j+1} .
$$

This holds because we fix our setting to strictly increasing functions. We only prove the case $\widetilde{x}_{i_{2}} \in \operatorname{hyp}_{\mathrm{S}}\left(f_{i}\right)$; see Figure $4.5 \mathrm{a}$ for an illustration. The case $\widetilde{x}_{i_{2}} \in \operatorname{epi}_{\mathrm{S}}\left(f_{i}\right)$ can be shown analogously; see Figure $4.5 \mathrm{~b}$ for an illustration. We prove $\stackrel{\circ}{x}_{i} \in \underline{R}\left(\widetilde{x}_{i}\right)$. Let $x_{i}^{\prime} \in \operatorname{gr}\left(f_{i}\right) \backslash \underline{R}\left(\widetilde{x}_{i}\right)$ with $\widetilde{x}_{i_{1}}<x_{i_{1}}^{\prime}$; see Figure $4.5 \mathrm{a}$ again. Strict monotonicity of $f_{i}$ and $\widetilde{x}_{i_{2}}<f_{i}\left(\widetilde{x}_{i_{1}}\right)$ imply $\widetilde{x}_{i_{2}}<f_{i}\left(\widetilde{x}_{i_{1}}\right)<f_{i}\left(x_{i_{1}}^{\prime}\right)=x_{i_{2}}^{\prime}$ and we obtain

$$
\left\|\widetilde{x}_{i}-\left(\widetilde{x}_{i_{1}}, f_{i}\left(\widetilde{x}_{i_{1}}\right)\right)\right\|_{2}^{2}<\left\|\widetilde{x}_{i}-x_{i}^{\prime}\right\|_{2}^{2} .
$$

This implies that the subproblem's objective value at $\left(\widetilde{x}_{i_{1}}, f_{i}\left(\widetilde{x}_{i_{1}}\right)\right) \in \underline{R}\left(\widetilde{x}_{i}\right)$ is smaller than at $x_{i}^{\prime} \notin \underline{R}\left(\widetilde{x}_{i}\right)$. The same holds for every $x_{i}^{\prime} \in \operatorname{gr}\left(f_{i}\right) \backslash \underline{R}\left(\widetilde{x}_{i}\right)$ with $x_{i_{1}}^{\prime}<f_{i}^{-1}\left(\widetilde{x}_{i_{2}}\right)$. This shows $\stackrel{\circ}{x}_{i} \in \underline{R}\left(\widetilde{x}_{i}\right)$ and yields

$$
{\stackrel{\circ}{x_{1}}}_{i_{1}} \leq \widetilde{x}_{i_{1}} \text { and } \quad \widetilde{x}_{i_{2}} \leq \stackrel{\circ}{x}_{i_{2}}
$$

From (4.8a) now follows

$$
\stackrel{\circ}{x}_{i_{1}}<x_{i_{1}}^{j+1} \text { and } x_{i_{2}}^{j}<\stackrel{\circ}{x}_{i_{2}}
$$

and strict monotonicity of $f_{i}$ finally implies

$$
x_{i_{1}}^{j}<{\stackrel{\circ}{x_{i}}}_{i_{1}}<x_{i_{1}}^{j+1} \text { and } x_{i_{2}}^{j}<\stackrel{\circ}{x}_{i_{2}}<x_{i_{2}}^{j+1} .
$$

Part (a) of Lemma 4.4.4 states that $\psi_{i}\left(\widetilde{x}_{i}\right)=0$ holds if the solution of the master problem is a linearization point for $i \in[\sigma]$. In this case, we obtain $\ell_{2}-\varepsilon$-feasibility for this $i \in[\sigma]$. Thus, we only consider the case $\psi_{i}\left(\widetilde{x}_{i}\right)>\varepsilon>0$ in the following. Lemma 4.4 .4 (b) states that the solution of the master problem and its corresponding subproblem's solution 


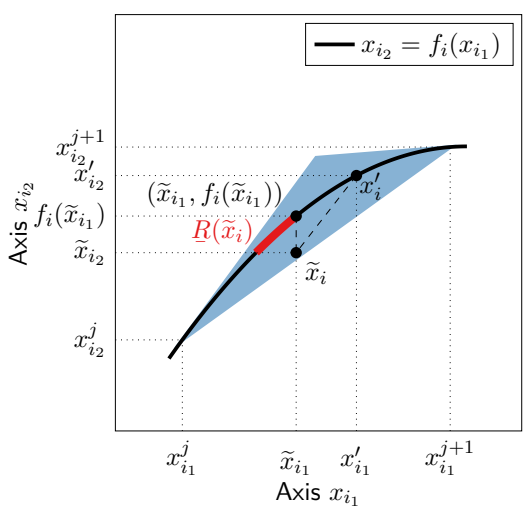

(a) Case $\widetilde{x}_{i} \in \operatorname{hyps}\left(f_{i}\right)$.

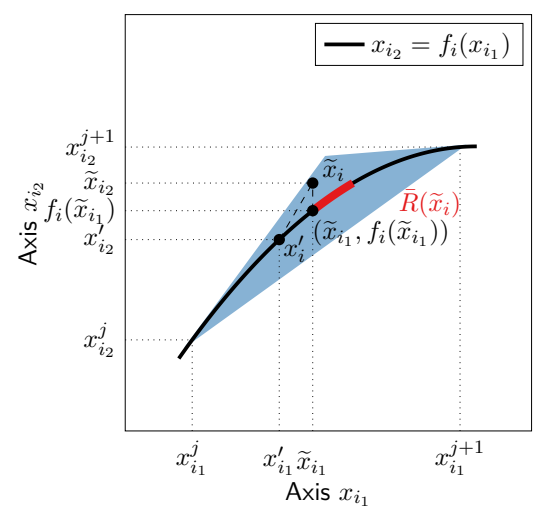

(b) Case $\widetilde{x}_{i} \in \operatorname{epi}_{\mathrm{S}}\left(f_{i}\right)$.

Figure 4.5: Illustration of the proof of Lemma 4.4.4 (b).

lie between the same linearization points; see (4.8a) and (4.8b). We exploit this situation in the following.

Before we state and prove Theorem 4.4.6, i.e., the statement that a solution $\widetilde{x}_{i}$ of the master problem with $\psi_{i}\left(\widetilde{x}_{i}\right)>\varepsilon>0$ is always cut off in the subsequent master problems, we make a proposition that is needed for the proof.

Proposition 4.4.5. Let $\widetilde{x}_{i} \in \Omega_{i}$ be part of a solution of the master problem $(\mathrm{M}(k))$ with $\widetilde{x}_{i} \in \operatorname{epi}\left(f_{i}\right)$ and let $\stackrel{\stackrel{x}{x}}{i}$ be part of the solution of the subsequent subproblem $(\mathrm{S}(i, k))$ with $\psi_{i}\left(\widetilde{x}_{i}\right)>\varepsilon>0$ for an $i \in[\sigma]$. Then $\stackrel{\circ}{x}_{i}$ is the orthogonal projection of $\widetilde{x}_{i}$ onto $\operatorname{gr}\left(f_{i}\right)$, i.e., on the tangent

$$
x_{i_{2}}=f_{i}\left(\stackrel{\circ}{x}_{i_{1}}\right)+f_{i}^{\prime}\left(\stackrel{\circ}{x}_{i_{1}}\right)\left(x_{i_{1}}-\stackrel{\circ}{x}_{i_{1}}\right) .
$$

Theorem 4.4.6. Let $\Omega_{i}$ be defined by $\mathcal{L}_{i}, \mathcal{C}_{i}$, and $\mathcal{G}_{i}$ and let $\widetilde{x}_{i} \in \Omega_{i}$ be part of a solution of the master problem $(\mathrm{M}(k))$ and $\stackrel{\circ}{x}_{i}$ be part of a solution of the subsequent subproblem $(\mathrm{S}(i, k))$ with $\psi_{i}\left(\widetilde{x}_{i}\right)>\varepsilon>0$ for an $i \in[\sigma]$. Then $\widetilde{x}_{i} \notin \Omega_{i}^{\prime}$ where $\Omega_{i}^{\prime}$ is defined by $\mathcal{L}_{i}^{\prime}:=\mathcal{L}_{i} \cup\left\{\stackrel{\circ}{x}_{i_{1}}\right\}, \mathcal{C}_{i}^{\prime}:=\mathcal{C}_{i} \cup\left\{\stackrel{\circ}{x}_{i_{2}}\right\}$, and $\mathcal{G}_{i}^{\prime}:=\mathcal{G}_{i} \cup\left\{f^{\prime}\left(\stackrel{\leftrightarrow}{x}_{i_{1}}\right)\right\}$.

Proof. We distinguish between the two cases $\widetilde{x}_{i} \in \operatorname{epi}_{\mathrm{S}}\left(f_{i}\right)$ and $\widetilde{x}_{i} \in \operatorname{hyp}{ }_{\mathrm{S}}\left(f_{i}\right)$ and start with the former. The outer approximation cut for $f_{i}$ at $\stackrel{\circ}{x}_{i}$ as given in constraint $(4.7 \mathrm{~b})$ can be written as $\bar{n}_{i}^{\top} x_{i} \leq \bar{\nu}_{i}$ with $\bar{n}_{i}=\left(-f_{i}^{\prime}\left({\stackrel{\circ}{x_{1}}}_{i_{1}}\right), 1\right)^{\top}$ and $\bar{\nu}_{i}=\stackrel{\circ}{x}_{i_{2}}-f_{i}^{\prime}\left(\stackrel{\circ}{x}_{i_{1}}\right) \stackrel{\circ}{x}_{i_{1}}$ where $\bar{n}_{i}$ is the normal vector and $\bar{n}_{i}^{\top} \stackrel{\circ}{x}_{i}=\bar{\nu}_{i}$. Proposition 4.4.5 yields that $\stackrel{\circ}{x}_{i}$ is the unique orthogonal projection of $\widetilde{x}_{i}$ onto $\operatorname{gr}\left(f_{i}\right)$. Consequently, there is some $c_{i} \in \mathbb{R}_{>0}$ with $\widetilde{x}_{i}-\stackrel{\circ}{x}_{i}=c_{i} \bar{n}_{i}$. 


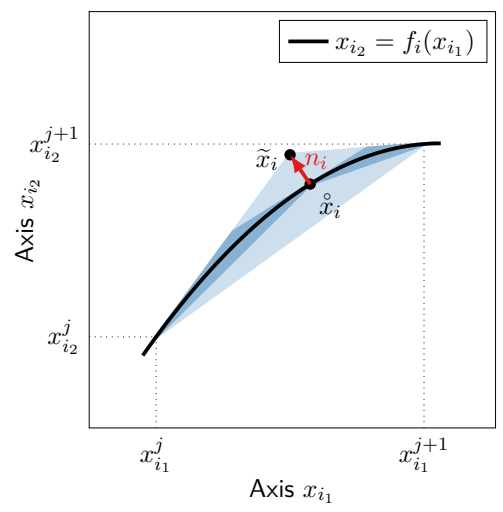

(a) Case $\widetilde{x}_{i} \in \operatorname{epis}\left(f_{i}\right)$.

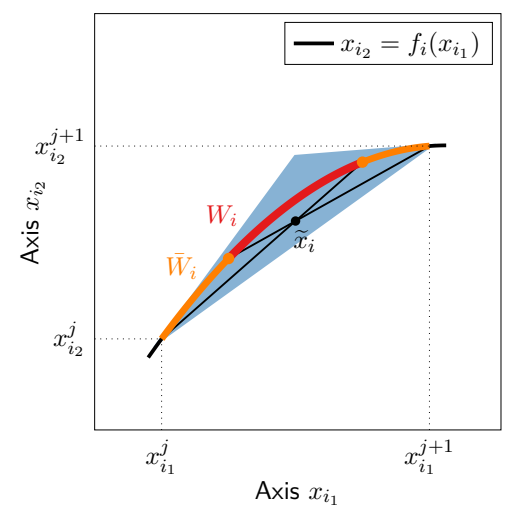

(b) Case $\widetilde{x}_{i} \in \operatorname{hyps}\left(f_{i}\right)$.

Figure 4.6: Illustration of the proof of Theorem 4.4.6.

Multiplication of the tangent with $c_{i}$ yields $n_{i}^{\top} x_{i} \leq \nu_{i}$ with $n_{i}=\widetilde{x}_{i}-\stackrel{\circ}{x}_{i}$ and $\nu_{i}=c_{i} \bar{\nu}_{i}$. Obviously, $\operatorname{hyp}\left(f_{i}\right)$ is strictly convex as $f_{i}$ is strictly concave. Hence, we have $\left(\stackrel{\circ}{x}_{i}-\widetilde{x}_{i}\right)^{\top}\left(x_{i}^{\text {hyp }}-\stackrel{\circ}{x}_{i}\right) \geq 0$ for all $x_{i}^{\text {hyp }} \in \operatorname{hyp}\left(f_{i}\right)$ because $\widetilde{x}_{i} \notin \operatorname{hyp}\left(f_{i}\right)$. This implies

$$
n_{i}^{\top} x_{i}^{\text {hyp }} \leq n_{i}^{\top} \stackrel{\circ}{x}_{i}=c_{i} \bar{n}_{i}^{\top} \stackrel{\circ}{x}_{i}=c_{i} \bar{\nu}_{i}=\nu_{i} .
$$

Thus, $n_{i}^{\top} x_{i} \leq \nu_{i}$ is a valid cut for $\operatorname{gr}\left(f_{i}\right)$ as $\operatorname{gr}\left(f_{i}\right) \subseteq \operatorname{hyp}\left(f_{i}\right)$. On the other hand,

$$
n_{i}^{\top} \widetilde{x}_{i}=n_{i}^{\top} \stackrel{\circ}{x}_{i}+n_{i}^{\top}\left(\widetilde{x}_{i}-\stackrel{\circ}{x}_{i}\right)=\nu_{i}+\left\|n_{i}\right\|_{2}^{2}>\nu_{i}
$$

holds. Hence, $n_{i}^{\top} x_{i} \leq \nu_{i}$ separates $\widetilde{x}_{i}$ and we have shown that $\widetilde{x}_{i} \notin \Omega_{i}^{\prime}$; see Figure 4.6a for an illustration.

Consider now the case $\widetilde{x}_{i} \in$ hyps $\left(f_{i}\right)$ and define $\Omega_{i}^{\prime}$ by integrating $\stackrel{\circ}{x}_{i}$ into the linearization. Lemma 4.4.4 (b) then yields

$$
\begin{aligned}
\mathcal{L}_{i}^{\prime} & =\left\{x_{i_{1}}^{0}, \ldots, x_{i_{1}}^{j}, \stackrel{\circ}{x}_{i_{1}}, x_{i_{1}}^{j+1}, \ldots, x_{i_{1}}^{r_{i}+1}\right\}, \\
\mathcal{C}_{i}^{\prime} & =\left\{x_{i_{2}}^{0}, \ldots, x_{i_{2}}^{j},{\stackrel{\circ}{i_{2}}}_{i_{2}}, x_{i_{2}}^{j+1}, \ldots, x_{i_{2}}^{r_{i}+1}\right\}, \\
\mathcal{G}_{i}^{\prime} & =\left\{f_{i}^{\prime}\left(x_{i_{1}}^{0}\right), \ldots, f_{i}^{\prime}\left(x_{i_{1}}^{j}\right), f_{i}^{\prime}\left(\stackrel{\circ}{x}_{i_{1}}\right), f_{i}^{\prime}\left(x_{i_{1}}^{j+1}\right), \ldots, f_{i}^{\prime}\left(x_{i_{1}}^{r_{i}+1}\right)\right\} .
\end{aligned}
$$

We define

$$
\begin{aligned}
& W\left(\widetilde{x}_{i}\right):=\left\{x_{i} \in \operatorname{gr}\left(f_{i}\right): \widetilde{x}_{i} \notin \Omega_{i}^{\prime \prime}, x_{i}^{j} \leq x_{i} \leq x_{i}^{j+1}\right\}, \\
& \bar{W}\left(\widetilde{x}_{i}\right):=\left\{x_{i} \in \operatorname{gr}\left(f_{i}\right) \backslash W\left(\widetilde{x}_{i}\right): x_{i}^{j} \leq x_{i} \leq x_{i}^{j+1}\right\},
\end{aligned}
$$


with $\Omega_{i}^{\prime \prime}$ defined by $\mathcal{L}_{i}^{\prime \prime}:=\mathcal{L}_{i} \cup\left\{x_{i_{1}}\right\}, \mathcal{C}_{i}^{\prime \prime}:=\mathcal{C}_{i} \cup\left\{x_{i_{2}}\right\}$, and $\mathcal{G}_{i}^{\prime \prime}:=\mathcal{G}_{i} \cup\left\{f_{i}^{\prime}\left(x_{i_{1}}\right)\right\}$. The set $W\left(\widetilde{x}_{i}\right)$ represents all solutions $\stackrel{\circ}{x}_{i}$ of the subproblem $(\mathrm{S}(i, k))$ that ensure that $\widetilde{x}_{i}$ is cut off in the next master problem; see Figure $4.6 \mathrm{~b}$ for an illustration. Thus, we have to show $\stackrel{\circ}{x}_{i} \in W\left(\widetilde{x}_{i}\right)$. As Lemma 4.4 .4 (b) ensures $\stackrel{\circ}{x}_{i} \in \underline{R}\left(\widetilde{x}_{i}\right)$, it is sufficient to prove $\underline{R}\left(\widetilde{x}_{i}\right) \subseteq W\left(\widetilde{x}_{i}\right)$. Assume $\stackrel{\circ}{x}_{i} \notin W\left(\widetilde{x}_{i}\right)$, i.e., $\stackrel{\circ}{x}_{i} \in \bar{W}\left(\widetilde{x}_{i}\right)$. This implies $\widetilde{x}_{i} \in \Omega_{i}^{\prime}$. We now consider the constraints $(4.7 \mathrm{c})$ and (4.7d) with $\delta_{i}^{o}=1$ for all $o \leq j, \delta_{i}^{j+1} \in[0,1]$, and $\delta_{i}^{o}=0$ for all $o \geq j+2$ resulting in

$$
\begin{aligned}
& \widetilde{x}_{i_{1}}=x_{i_{1}}^{j}+\left(\stackrel{\circ}{x}_{i_{1}}-x_{i_{1}}^{j}\right) \delta_{i}^{j^{*}}+\left(x_{i_{1}}^{j+1}-\stackrel{\leftrightarrow}{x}_{i_{1}}\right) \delta_{i}^{j+1}, \\
& \widetilde{x}_{i_{2}} \geq x_{i_{2}}^{j}+\left(\stackrel{\circ}{x}_{i_{2}}-x_{i_{2}}^{j}\right) \delta_{i}^{j^{*}}+\left(x_{i_{2}}^{j+1}-\stackrel{\circ}{x}_{i_{2}}\right) \delta_{i}^{j+1} .
\end{aligned}
$$

Here, $j^{*}$ is the new index of the incremental method after adding $\stackrel{\circ}{x}_{i}$ to $\mathcal{L}_{i}^{\prime}, \mathcal{C}_{i}^{\prime}$, and $\mathcal{G}_{i}^{\prime}$; see (4.9). Finally, we apply a case analysis:

(i) $\delta_{i}^{j^{*}}=\delta_{i}^{j+1}=0$. Equation (4.10) yields $\widetilde{x}_{i_{1}}=x_{i_{1}}^{j}$, which contradicts (4.8a) of Lemma 4.4.4.

(ii) $\delta_{i}^{j^{*}} \in(0,1), \delta_{i}^{j+1}=0$. Equation (4.10) yields $x_{i_{1}}^{j}<\widetilde{x}_{i_{1}}<\stackrel{\circ}{x}_{i_{1}}$; a contradiction because $\stackrel{\circ}{x}_{i} \in \underline{R}\left(\widetilde{x}_{i}\right)$ implies ${\stackrel{\circ}{x_{i}}}_{i_{1}} \leq \widetilde{x}_{i_{1}}$.

(iii) $\delta_{i}^{j^{*}}=1, \delta_{i}^{j+1}=0$. Equation (4.10) yields $\widetilde{x}_{i_{1}}={\stackrel{\circ}{x_{1}}}_{i_{1}}$ or $f_{i}\left(\widetilde{x}_{i_{1}}\right)=\stackrel{\circ}{x}_{i_{2}}$ and (4.11) yields $\widetilde{x}_{i_{2}} \geq{\stackrel{\circ}{i_{2}}}_{i_{2}}$. However, $\widetilde{x}_{i} \in \operatorname{hyp}_{\mathrm{S}}\left(f_{i}\right)$ implies $\widetilde{x}_{i_{2}}<f_{i}\left(\widetilde{x}_{i_{1}}\right)={\stackrel{\circ}{i_{2}}}_{i_{2}}$; again a contradiction.

(iv) $\delta_{i}^{j^{*}}=1, \delta_{i}^{j+1} \in(0,1)$. Equation (4.10) yields ${\stackrel{\circ}{i_{1}}}_{i_{1}}<\widetilde{x}_{i_{1}}<x_{i_{1}}^{j+1}$ and (4.11) yields $\widetilde{x}_{i_{2}} \geq{\stackrel{\circ}{x_{2}}}_{i_{2}}+\left(x_{i_{2}}^{j+1}-\stackrel{\circ}{x}_{i_{2}}\right) \delta_{i}^{j+1}>\stackrel{\circ}{x}_{i_{2}}$. Additionally, $\stackrel{\circ}{x}_{i} \in \underline{R}\left(\widetilde{x}_{i}\right)$ implies $\widetilde{x}_{i_{2}} \leq{\stackrel{\circ}{i_{2}}}_{i_{2}}$; a contradiction.

(v) $\delta_{i}^{j^{*}}=\delta_{i}^{j+1}=1$. In this case, (4.10) yields $\widetilde{x}_{i_{1}}=x_{i_{1}}^{j+1}$, which contra$\operatorname{dicts}(4.8 \mathrm{a})$.

This shows $\stackrel{\circ}{x}_{i} \in W\left(\widetilde{x}_{i}\right)$ and thus $\widetilde{x}_{i}$ is cut off, i.e., $\widetilde{x}_{i} \notin \Omega_{i}^{\prime}$; see Figure $4.6 \mathrm{~b}$.

We remark that the first case also follows from a suitable minimum principle; see, e.g., Mangasarian [113, Theorem 9.3.3]. See also Bonami et al. [17] for an application of the principle in a closely related context.

Theorem 4.4.7. Algorithm 4.1 terminates after a finite number of iterations with a globally optimal $\ell_{2}-\varepsilon$-feasible solution of (4.2) or with an indication that (4.2) is infeasible. 


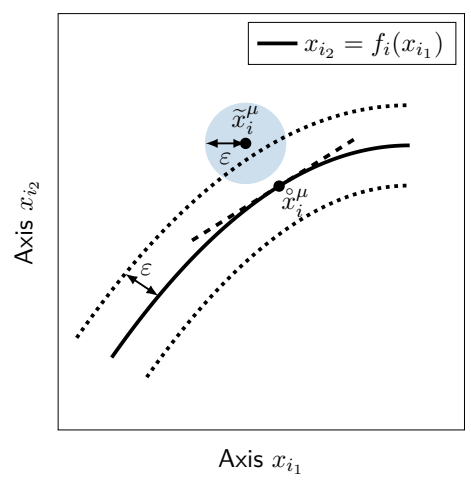

(a) Case $\widetilde{x}_{i} \in \operatorname{epis}\left(f_{i}\right)$.

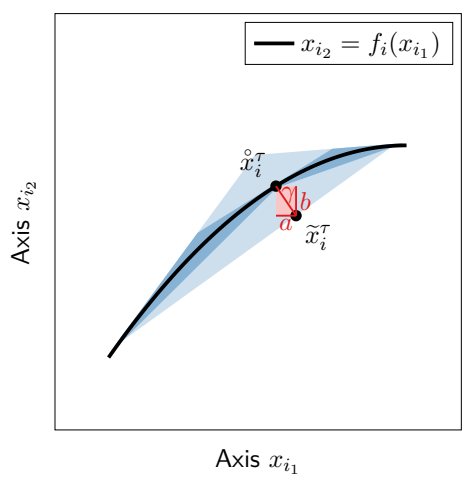

(b) Case $\widetilde{x}_{i} \in \operatorname{hyps}\left(f_{i}\right)$.

Figure 4.7: Illustration of the proof of Theorem 4.4.7.

Proof. We assume that the algorithm does not terminate after a finite number of iterations. Then there exists a sequence $\left(\widetilde{x}^{k}\right)_{k}$ of master problem's solutions such that all $(\mathrm{M}(k))$ are feasible and such that there exists at least one $i \in[\sigma]$ with $\psi_{i}\left(\widetilde{x}_{i}^{k}\right)>\varepsilon$ for all $k$. As there are only finitely many possible values in $[\sigma]$, there is at least one $i \in[\sigma]$ for which $\psi_{i}\left(\widetilde{x}_{i}^{k}\right)>\varepsilon$ infinitely many times. This subsequence has a further subsequence indexed by $\tau$ such that all $\tau$-iterates satisfy

$$
\tilde{x}_{i}^{\tau} \in \operatorname{epi}_{\mathrm{S}}\left(f_{i}\right) \quad \text { or } \quad \tilde{x}_{i}^{\tau} \in \operatorname{hyp}_{\mathrm{S}}\left(f_{i}\right) .
$$

We show that both cannot happen and start with the first case.

As all variables are bounded, the subsequence $\left(\widetilde{x}_{i}^{\tau}\right)_{\tau}$ of master problem solutions is bounded as well and we have a convergent subsequence $\left(\widetilde{x}_{i}^{\mu}\right)_{\mu}$. Thus, for every $\delta>0$ we have

$$
\left\|\widetilde{x}_{i}^{\alpha}-\widetilde{x}_{i}^{\beta}\right\|_{2}<\delta
$$

for all sufficiently large indices $\alpha$ and $\beta$ of the $\mu$-subsequence. On the other hand, all $\mu$-iterates are excluded by an outer approximation cut from the feasible set of the master problem in iteration $\tau+1$; see Theorem 4.4.6. This cut certainly excludes the $\varepsilon$-ball $B_{\varepsilon}\left(\widetilde{x}_{i}^{\mu}\right)$, which yields

$$
\left\|\widetilde{x}_{i}^{\alpha}-\widetilde{x}_{i}^{\beta}\right\|_{2}>\varepsilon>0
$$

for all $\alpha, \beta$; see Figure 4.7a for an illustration. This contradicts (4.12).

Next, we consider the case $\widetilde{x}_{i}^{\tau} \in \operatorname{hyp}_{\mathrm{S}}\left(f_{i}\right)$ for all iterates in the subsequence indexed by $\tau$. Strict monotonicity of $f_{i}$ implies that the rectangle spanned 
by the points $\widetilde{x}_{i}^{\tau}$ and $\stackrel{\circ}{x}_{i}^{\tau}$ is cut off from the feasible set of the master problem in iteration $\tau+1$ by the constraints (4.7c) and (4.7d). Let $\gamma>\varepsilon>0$ be the length of the diagonal of this rectangle and let $a$ and $b$ denote its sides. As $\stackrel{\circ}{x}_{i}^{\tau}$ is an orthogonal projection of $\widetilde{x}_{i}^{\tau}$ on $f_{i}$, the equality

$$
\frac{a}{b}=f_{i}^{\prime}\left(\stackrel{\circ}{x}_{i_{1}}^{\tau}\right)
$$

holds. Using the Pythagorean Theorem

$$
a^{2}+b^{2}=\gamma^{2}
$$

the values $a$ and $b$ can be written depending on $\gamma$ and $f_{i}^{\prime}\left(\stackrel{\circ}{x}_{i_{1}}^{\tau}\right)$ and so can the area of the rectangle with

$$
a b=\frac{\gamma f_{i}^{\prime}\left(\stackrel{\circ}{x}_{i_{1}}^{\tau}\right)}{\sqrt{1+f_{i}^{\prime}\left(\stackrel{\circ}{x}_{i_{1}}^{\tau}\right)^{2}}} \frac{\gamma}{\sqrt{1+f_{i}^{\prime}\left(\stackrel{\circ}{x}_{i_{1}}^{\tau}\right)^{2}}}=\frac{\gamma^{2} f_{i}^{\prime}\left(\stackrel{\circ}{x}_{i_{1}}^{\tau}\right)}{1+f_{i}^{\prime}\left(\stackrel{\circ}{x}_{i_{1}}^{\tau}\right)^{2}}>\gamma^{2} \xi>\varepsilon^{2} \xi
$$

for some $\xi>0$ as $f_{i}^{\prime}$ is both bounded above and away from zero. Remember that we require strict monotonicity of $f_{i}$. Consequently, we exclude $f_{i}^{\prime}\left(\stackrel{\circ}{x}_{i_{1}}^{\tau}\right)=0$ and $\xi=0$. However, the area of

$$
\operatorname{hyp}_{\mathrm{S}}\left(f_{i}\right) \cap\left[l_{i}, u_{i}\right]
$$

is finite, yielding a contradiction; see Figure 4.7b for an illustration.

\subsection{The Case of Lipschitz Continuous Functions and Exact Function Evaluations}

In this section, we assume that Assumption 4.2.2 holds. We present the details of the algorithm in regard to master problem $(\mathrm{M}(k))$ and subproblem $(\mathrm{S}(i, k))$ in Section 4.5.1. In Section 4.5.2, we then prove that the algorithm correctly terminates with a globally optimal $\varepsilon$-feasible point of Problem (4.2) or the indication of infeasibility. We remark that significant parts of this section can be found in our article Schmidt et al. [141]. Note that the following descriptions amplify the short and concise presentations of the article.

\subsubsection{Derivation of the Algorithm}

Master Problem Again, the master problem $(\mathrm{M}(k))$ is a relaxation of Problem (4.2) and is supposed to be an MIP. With Assumption 4.2.2, the 
functions $f_{i}$ are not necessary concave any more thus we cannot apply the approach from Section 4.4. Moreover, we cannot strengthen the bounds of the involved variables as this relies on strictly monotonic and strictly concave or convex functions. Once more, we assume that there are values $x_{i_{1}}^{k, j} \in \mathbb{R}$ with

$$
x_{i_{1}}^{k, 0}<x_{i_{1}}^{k, 1}<\cdots<x_{i_{1}}^{k, r_{i}^{k}}
$$

and $f_{i}\left(x_{i_{1}}^{k, j}\right)=: x_{i_{2}}^{k, j} \in \mathbb{R}$ for $j \in\left[r_{i}^{k}\right]$. Again, we abbreviate

$$
\begin{aligned}
\mathcal{L}_{i}^{k} & :=\left\{x_{i_{1}}^{-}=: x_{i_{1}}^{k, 0}, x_{i_{1}}^{k, 1}, \ldots, x_{i_{1}}^{k, r_{i}^{k}-1}, x_{i_{1}}^{k, r_{i}^{k}}:=x_{i_{1}}^{+}\right\}, \\
\mathcal{C}_{i}^{k} & :=\left\{f_{i}\left(x_{i_{1}}^{-}\right)=: x_{i_{2}}^{k, 0}, x_{i_{2}}^{k, 1}, \ldots, x_{i_{2}}^{k, r_{i}^{k}-1}, x_{i_{2}}^{k, r_{i}^{k}}:=f_{i}\left(x_{i_{1}}^{+}\right)\right\} .
\end{aligned}
$$

We know the global Lipschitz constant $L_{i}$ of $f_{i}$ on $\left[x_{i_{1}}^{-}, x_{i_{1}}^{+}\right]$for all $i \in[\sigma]$ according to Assumption 4.2.2. Thus, we can construct a sequence of quadrilaterals as a relaxation of $\operatorname{gr}\left(f_{i}\right) \cap\left[x_{i}^{-}, x_{i}^{+}\right]$. We define the quadrilaterals $\Omega_{i}^{k}(j)$ for $j \in\left[r_{i}^{k}-1\right]$ as

$$
\begin{aligned}
\Omega_{i}^{k}(j):=\{ & \left(x_{i_{1}}, x_{i_{2}}\right) \in \mathbb{R}^{2}: x_{i_{1}}^{k, j} \leq x_{i_{1}} \leq x_{i_{1}}^{k, j+1}, \\
& x_{i_{2}} \leq x_{i_{2}}^{k, j}+L_{i}\left(x_{i_{1}}-x_{i_{1}}^{k, j}\right) \\
& x_{i_{2}} \geq x_{i_{2}}^{k, j}-L_{i}\left(x_{i_{1}}-x_{i_{1}}^{k, j}\right) \\
& x_{i_{2}} \leq x_{i_{2}}^{k, j+1}+L_{i}\left(x_{i_{1}}^{k, j+1}-x_{i_{1}}\right) \\
& \left.x_{i_{2}} \geq x_{i_{2}}^{k, j+1}-L_{i}\left(x_{i_{1}}^{k, j+1}-x_{i_{1}}\right)\right\}
\end{aligned}
$$

and obtain $\Omega_{i}^{k}$ for all $i \in[\sigma]$ by (4.6). The set $\Omega_{i}^{k}$ for the $k$ th master problem $(\mathrm{M}(k))$ is uniquely defined by the sets $\mathcal{L}_{i}^{k}$ and $\mathcal{C}_{i}^{k}$ and depicted in Figure 4.8b. Compared to Section 4.4, every single $\Omega_{i}^{k}(j)$ for all $j \in\left[r_{i}^{k}-1\right]$ is a quadrilateral instead of a triangle. Analogously to Proposition 4.4.1, we formulate a proposition directly by construction. 


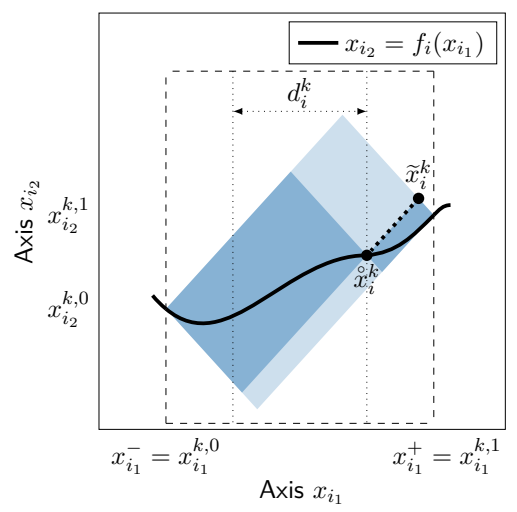

(a) Subproblem $\left(\mathrm{S}^{\prime}(i, k)\right)$ for an $i \in[\sigma]$.

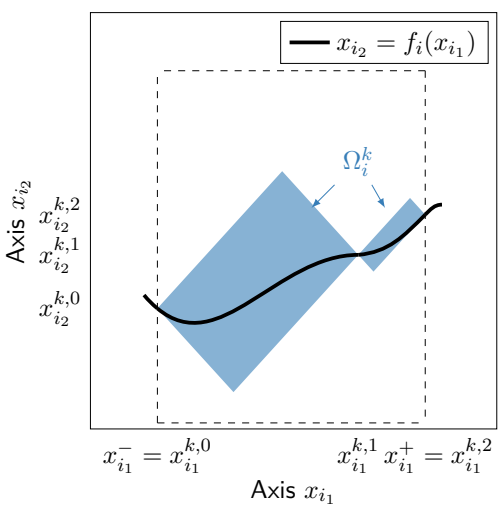

(b) Set $\Omega_{i}^{k}$ of master problem $(\mathrm{M}(k))$ for an $i \in[\sigma]$.

Figure 4.8: Setting for subproblem and master problem for Algorithm 4.2.

Proposition 4.5.1. For iteration $k$, we define $\Omega_{i}^{k}$ with (4.6) and (4.13) for all $i \in[\sigma]$. Then

$$
\Omega_{i}^{k} \supseteq \operatorname{gr}\left(f_{i}\right) \cap\left[x_{i}^{-}, x_{i}^{+}\right]
$$

holds. Thus, the kth master problem $(\mathrm{M}(k))$ is a relaxation of (4.2).

Instead of Proposition 4.4.2, we formulate a lemma.

Lemma 4.5.2. For iteration $k$, we define $\Omega_{i}^{k}$ with (4.6) and (4.13) for all $i \in[\sigma]$. The master problem $(\mathrm{M}(k))$ is an MIP.

Proof. The constraints $A x \geq b, x^{-} \leq x \leq x^{+}, x_{\mathcal{C}} \in \mathbb{R}^{|\mathcal{C}|}$, and $x_{\mathcal{I}} \in \mathbb{Z}^{|\mathcal{I}|}$ are mixed-integer linear constraints. It remains to prove that $x_{i} \in \Omega_{i}^{k}$ can be formulated as an MIP model as well. The sets $\Omega_{i}^{k}$ are finite unions of polytopes $\Omega_{i}^{k}(j)$; see (4.6) and (4.13). Unions of polytopes can be modeled using Big- $M$ constraints yielding the MIP-formulation

$$
\begin{array}{r}
-M\left(1-\omega_{i}^{k, j}\right)+x_{i_{1}}^{k, j} \leq x_{i_{1}} \leq x_{i_{1}}^{k, j+1}+M\left(1-\omega_{i}^{k, j}\right) \\
\text { for all } j \in\left[r_{i}^{k}-1\right], \\
x_{i_{2}} \leq x_{i_{2}}^{k, j}+L_{i}\left(x_{i_{1}}-x_{i_{1}}^{k, j}\right)+M\left(1-\omega_{i}^{k, j}\right) \\
\text { for all } j \in\left[r_{i}^{k}-1\right], \\
x_{i_{2}} \geq x_{i_{2}}^{k, j}-L_{i}\left(x_{i_{1}}-x_{i_{1}}^{k, j}\right)-M\left(1-\omega_{i}^{k, j}\right) \\
\quad \text { for all } j \in\left[r_{i}^{k}-1\right], \\
x_{i_{2}} \leq x_{i_{2}}^{k, j+1}+L_{i}\left(x_{i_{1}}^{k, j+1}-x_{i_{1}}\right)+M\left(1-\omega_{i}^{k, j}\right)
\end{array}
$$




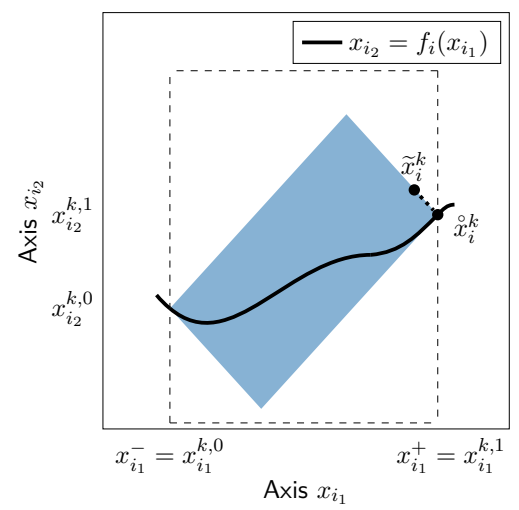

Figure 4.9: Using subproblem $(\mathrm{S}(i, k))$ fails for Algorithm 4.2.

$$
\begin{aligned}
& \text { for all } j \in\left[r_{i}^{k}-1\right] \text {, } \\
& x_{i_{2}} \geq x_{i_{2}}^{k, j+1}-L_{i}\left(x_{i_{1}}^{k, j+1}-x_{i_{1}}\right)-M\left(1-\omega_{i}^{k, j}\right) \\
& \text { for all } j \in\left[r_{i}^{k}-1\right] \text {, } \\
& \sum_{j \in\left[r_{i}^{k}-1\right]} \omega_{i}^{k, j}=1, \\
& \omega_{i}^{k, j} \in\{0,1\} \quad \text { for all } j \in\left[r_{i}^{k}-1\right],
\end{aligned}
$$

for all $\Omega_{i}^{k}$ with $i \in[\sigma]$ in the $k$ th master problem. The additional binary variables $\omega$ in $(4.14 \mathrm{~g}$ ) (de)activate polytopes and their corresponding linear constraints (4.14a)-(4.14e). SOS-1 constraints (4.14f) ensure that only one polytope is activated.

The general situation for the master problem $(\mathrm{M}(k))$ as an MIP is the same as before in Section 4.4.

Subproblem Again, given a solution $\widetilde{x}_{i}^{k}$ of $(\mathrm{M}(k))$, a subproblem is solved to provide a new point $\stackrel{\circ}{x}_{i}^{k}$ to be added to $\mathcal{L}_{i}^{k+1}$ and $\mathcal{C}_{i}^{k+1}$. Besides that, in Section 4.4, we solve the subproblem to detect $\ell_{2}$ - $\varepsilon$-feasibility of the solution found by $(\mathrm{M}(k))$. The situation is different now and we have to adjust the subproblem.

Figure 4.9 shows a possible problem with subproblem $(\mathrm{S}(i, k))$ in the setting of Section 4.4. The subproblem's solution $\stackrel{\circ}{x}_{i}^{k}$ delivers an already known linearization point. Therefore, the master problem solves the same problem again. The algorithm is caught in an endless loop and we cannot show finite termination. 
To assert that the newly found points do not accumulate at an already known value, we reformulate the subproblem. For a given $j \in\left[r_{i}^{k}-1\right]$, we define the sets

$$
\stackrel{\circ}{\Omega}_{i}^{k}(j):=\Omega_{i}^{k}(j) \cap\left\{\left(x_{1}, x_{2}\right) \in \mathbb{R}^{2} \mid x_{i_{1}}^{k, j}+0.25 d_{i}^{k, j} \leq x_{i_{1}} \leq x_{i_{1}}^{k, j+1}-0.25 d_{i}^{k, j}\right\}
$$

for all $i \in[\sigma]$ where $d_{i}^{k, j}=x_{i_{1}}^{k, j+1}-x_{i_{1}}^{k, j}$ is the length of the corresponding subinterval. Note that the constant 0.25 can be replaced by any other constant in $(0,1 / 2)$. The new subproblem now reads

$$
\begin{array}{ll}
\min _{x_{i}} & \left\|x_{i}-\widetilde{x}_{i}^{k}\right\|_{2}^{2} \\
\text { s.t. } & x_{i} \in \operatorname{gr}\left(f_{i}\right), \\
& x_{i} \in \stackrel{\circ}{\Omega}_{i}^{k}\left(j_{i}^{k}\right),
\end{array}
$$

where $j_{i}^{k}$ denotes the quadrilateral of the corresponding master problem's solution $\widetilde{x}_{i}^{k}$; see Figure 4.8a for an illustration. This can easily be implemented by using the binary variables $\omega_{i}^{k, j}$ of the MIP formulation (4.14). Note that Figure $4.8 \mathrm{~b}$ shows the master problem with the result of the subproblem in Figure 4.8a. By using subproblem $\left(\mathrm{S}^{\prime}(i, k)\right)$, we ensure solutions between two linearization points that do not accumulate at an already known value; see Figure 4.8a. In contrast to Section 4.4, where the nonempty feasible set of the subproblem $(\mathrm{S}(k))$ follows by construction, we have to show this property now.

Lemma 4.5.3. For iteration $k$, the subproblems $\left(\mathrm{S}^{\prime}(i, k)\right)$ are feasible for all $i \in[\sigma]$.

Proof. The claim follows by Lipschitz continuity and the definition of the sets $\stackrel{\Omega}{i}_{i}^{k}\left(j_{i}^{k}\right)$.

As the objective values of subproblem $\left(\mathrm{S}^{\prime}(i, k)\right)$ have no meaning for the feasibility of the master problem's solution, we use $\varepsilon$-feasibility to decide feasibility; see Definition 4.3.3. This is possible as an oracle exists; see Assumption 4.2.2.

Decomposition Algorithm We are now able to formally state the decomposition algorithm for solving Problem (4.2) in case of Assumption 4.2.2; see Algorithm 4.2. After the master problem is solved in Line 3, the algorithm checks whether we possess an $\varepsilon$-feasible point in Line 8 . The determination of the indices $j_{i}^{k}$ using the binary variables $\omega_{i}^{k, j}$ of the MIP 
Algorithm 4.2 Decomposition Method in Case of Assumption 4.2.2

Input: Problem (4.2) and $\varepsilon>0$.

Output: If there is an $\varepsilon$-feasible point for Problem (4.2), the algorithm returns a globally optimal $\varepsilon$-feasible solution $\widetilde{x}$. Otherwise, it returns an indication of infeasibility.

1: Set $k \leftarrow 1$ and define $\mathcal{L}_{i}^{k}:=\left\{x_{i_{1}}^{-}, x_{i_{1}}^{+}\right\}, \mathcal{C}_{i}^{k}:=\left\{f_{i}\left(x_{i_{1}}^{-}\right), f_{i}\left(x_{i_{1}}^{+}\right)\right\}$for all $i \in[\sigma]$.

2: while true do

3: $\quad$ Solve master problem $(\mathrm{M}(k))$ with $\mathcal{L}_{i}^{k}$ and $\mathcal{C}_{i}^{k}$ to global optimality where $\Omega_{i}^{k}$ is defined by (4.14) for all $i \in[\sigma]$.

4: $\quad$ if master problem $(\mathrm{M}(k))$ is infeasible then

5: return "Problem (4.2) is infeasible".

6: end if

7: $\quad$ Denote the solution of master problem $(\mathrm{M}(k))$ by $\widetilde{x}^{k}$.

8: $\quad$ if $\left|f_{i}\left(\widetilde{x}_{i_{1}}^{k}\right)-\widetilde{x}_{i_{2}}^{k}\right| \leq \varepsilon$ for all $i \in[\sigma]$ then

9: $\quad$ return globally optimal $\varepsilon$-feasible solution $\widetilde{x}^{k}$.

10: end if

11: for $i \in[\sigma]$ do

12: $\quad$ if $\left|f_{i}\left(\widetilde{x}_{i_{1}}^{k}\right)-\widetilde{x}_{i_{2}}^{k}\right|>\varepsilon$ then

13: $\quad$ Determine the polytope $j_{i}^{k} \in\left[r_{k}^{i}-1\right]$ out of the master problem's solution $\widetilde{x}_{i}^{k}$.

14: $\quad$ Solve subproblems $\left(\mathrm{S}^{\prime}(i, k)\right)$ yielding solutions $\stackrel{\circ}{x}_{i}^{k}$ and set $\mathcal{L}_{i}^{k+1} \leftarrow \mathcal{L}_{i}^{k} \cup\left\{\stackrel{\circ}{x}_{i_{1}}^{k}\right\}$ and $\mathcal{C}_{i}^{k+1} \leftarrow \mathcal{C}_{i}^{k} \cup\left\{\stackrel{\circ}{x}_{i_{2}}^{k}\right\}$

15: $\quad$ else

16: $\quad$ Set $\mathcal{L}_{i}^{k+1} \leftarrow \mathcal{L}_{i}^{k}$ and $\mathcal{C}_{i}^{k+1} \leftarrow \mathcal{C}_{i}^{k}$

17: end if

18: end for

19: $\quad$ Increase $k \leftarrow k+1$.

20: end while

formulation (4.14) happens in Line 13. If an $\varepsilon$-feasible point is not obtained, there are subproblems with feasibility violation larger than $\varepsilon$. For these indices $i$, we then refine the relaxation by adding the corresponding subproblem's solution $\stackrel{\circ}{x}_{i_{1}}^{k}$ and $\stackrel{\circ}{x}_{i_{2}}^{k}$ to the sets $\mathcal{L}_{i}^{k}$ and $\mathcal{C}_{i}^{k}$, respectively in Line 14 . The algorithm returns a globally optimal $\varepsilon$-feasible point of Problem (4.2) or the indication of infeasibility. 


\subsubsection{Convergence Results}

Again, we prove that Algorithm 4.2 is correct in the sense that it terminates with a globally optimal $\varepsilon$-feasible point of Problem (4.2) or with the indication of infeasibility. We show that in Theorem 4.5.5. Beforehand, we state and prove a lemma and introduce some more notation. The sets $\mathcal{L}_{i}^{k}$ and $\mathcal{C}_{i}^{k}$ define the unions of polytopes $\Omega_{i}^{k}$ for all $i \in[\sigma]$. If the former sets are updated in Algorithm 4.2, we have $\mathcal{L}_{i}^{k+1} \leftarrow \mathcal{L}_{i}^{k} \cup\left\{\stackrel{\circ}{x}_{i_{1}}^{k}\right\}$ and $\mathcal{C}_{i}^{k+1} \leftarrow \mathcal{C}_{i}^{k} \cup\left\{\stackrel{\circ}{x}_{i_{2}}^{k}\right\}$. This update corresponds to the refinement of one quadrilateral in $\Omega_{i}^{k}$. To be more specific, the quadrilateral $j_{i}^{k}$ is replaced by two smaller ones that we denote by $\Omega_{i}^{k}\left(j_{\alpha}^{k}\right)$ and $\Omega_{i}^{k}\left(j_{\beta}^{k}\right)$ obtaining

$$
\Omega_{i}^{k+1}=\Omega_{i}^{k}\left(j_{\alpha}^{k}\right) \bigcup \Omega_{i}^{k}\left(j_{\beta}^{k}\right) \bigcup_{j \neq j_{i}^{k} \in\left[r_{i}^{k}-1\right]} \Omega_{i}^{k}(j) .
$$

We first prove an auxiliary result.

Lemma 4.5.4. There exists a constant $\Lambda>0$ depending on $\varepsilon$ and $L$ alone such that as long as Algorithm 4.2 does not terminate in Line 5 or 9, there exists an $\Lambda^{k}>\Lambda$ for every iteration $k$ such that

$$
\operatorname{Vol}\left(\Omega_{i}^{k}\left(j_{\alpha}^{k}\right)\right)+\operatorname{Vol}\left(\Omega_{i}^{k}\left(j_{\beta}^{k}\right)\right)=\operatorname{Vol}\left(\Omega_{i}^{k}\left(j_{i}^{k}\right)\right)-\Lambda^{k}
$$

holds for at least one $i \in[\sigma]$.

Proof. Algorithm 4.2 does not terminate in Line 9 by construction. Thus, there is an $i \in[\sigma]$ with a solution $\left(\widetilde{x}_{i_{1}}^{k}, \widetilde{x}_{i_{2}}^{k}\right) \in \Omega_{i}^{k}\left(j_{i}^{k}\right)$ and

$$
\left|f_{i}\left(\widetilde{x}_{i_{1}}^{k}\right)-\widetilde{x}_{i_{2}}^{k}\right|>\varepsilon .
$$

We calculate the initial volume of $\Omega_{i}^{k}\left(j_{i}^{k}\right)$ and prove the volume reduction (4.15) after inserting the linearization point $\left(\stackrel{\circ}{x}_{i_{1}}^{k}, \stackrel{\circ}{x}_{i_{2}}^{k}\right)$ in Line 14. In order to do so, we calculate the corners (left, right, bottom, top) of $\Omega_{i}^{k}\left(j_{i}^{k}\right)$ that are given in $\left(x_{i_{1}}, x_{i_{2}}\right)$-coordinates as

$$
\begin{aligned}
& \mathbf{l}=\left(a, f_{i}(a)\right)^{\top}, \\
& \mathbf{r}=\left(b, f_{i}(b)\right)^{\top} \\
& \mathbf{b}=\left(\frac{f_{i}(a)-f_{i}(b)+L(b+a)}{2 L}, \frac{f_{i}(a)+f_{i}(b)+L(b-a)}{2}\right)^{\top}, \\
& \mathbf{t}=\left(\frac{f_{i}(b)-f_{i}(a)+L(b+a)}{2 L}, \frac{f_{i}(a)+f_{i}(b)+L(b-a)}{2}\right)^{\top},
\end{aligned}
$$


4.5 The Case of Lipschitz Continuous Functions and Exact Function Evaluations

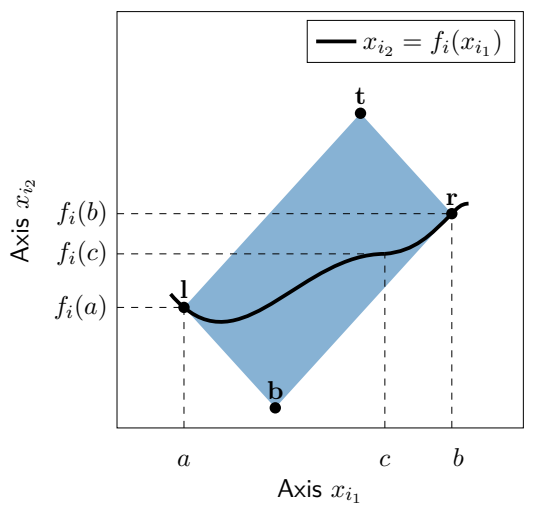

Figure 4.10: Illustration of the proof of Lemma 4.5.4.

where $a=x_{i_{1}}^{k, j_{i}^{k}}$ and $b=x_{i_{2}}^{k, j_{i}^{k}+1}$; see Figure 4.10. The volume of $\Omega_{i}^{k}\left(j_{i}^{k}\right)$ is now given as

$$
\begin{aligned}
\operatorname{Vol}\left(\Omega_{i}^{k}\left(j_{i}^{k}\right)\right) & =|\operatorname{det}(\mathbf{t}-\mathbf{l} \mathbf{b}-\mathbf{l})| \\
& =\left|L \frac{(b-a)^{2}}{2}-\frac{\left(f_{i}(b)-f_{i}(a)\right)^{2}}{2 L}\right| \\
& =L \frac{(b-a)^{2}}{2}-\frac{\left(f_{i}(b)-f_{i}(a)\right)^{2}}{2 L} .
\end{aligned}
$$

We remark that the calculation of the determinant is straightforward. The last equality holds because of Lipschitz continuity. The maximal $x_{i_{2}}$-distance in $\Omega_{i}^{k}\left(j_{i}^{k}\right)$ is obtained over the point $\mathbf{b}$ (or below $\mathbf{t}$ ) and is given as

$$
-\left|f_{i}(b)-f_{i}(a)\right|+L(b-a)
$$

Again, the calculation of the distance is straightforward. Together with the fact that there is an infeasible point according to (4.16), the inequality

$$
-\left|f_{i}(b)-f_{i}(a)\right|+L(b-a)>\varepsilon
$$

holds and thus we conclude

$$
L(b-a)>\varepsilon
$$

The new intersection point $c={\stackrel{\circ}{i_{1}}}_{k}^{k}$ satisfies $c \in[a+0.25 d, b-0.25 d]$ with

$$
d=b-a \text {. }
$$


4 Decomposition Methods for Mixed-Integer Nonlinear Programs

and thus

$$
b-c \geq \frac{d}{4} \quad \text { and } \quad c-a \geq \frac{d}{4}
$$

holds. Analogous calculations now show that the volumes of the new quadrilaterals fulfill

$$
\begin{aligned}
& \operatorname{Vol}\left(\Omega_{i}^{k}\left(j_{\alpha}^{k}\right)\right)=L \frac{(c-a)^{2}}{2}-\frac{\left(f_{i}(c)-f_{i}(a)\right)^{2}}{2 L} \\
& \operatorname{Vol}\left(\Omega_{i}^{k}\left(j_{\beta}^{k}\right)\right)=L \frac{(b-c)^{2}}{2}-\frac{\left(f_{i}(b)-f_{i}(c)\right)^{2}}{2 L} .
\end{aligned}
$$

A straightforward calculation gives

$$
\begin{aligned}
\operatorname{Vol}\left(\Omega_{i}^{k}\left(j_{i}^{k}\right)\right)= & L \frac{(b-c+c-a)^{2}}{2}-\frac{\left(f_{i}(b)-f_{i}(c)+f_{i}(c)-f_{i}(a)\right)^{2}}{2 L} \\
= & L \frac{(b-c)^{2}+2(b-c)(c-a)+(c-a)^{2}}{2} \\
& -\frac{\left(f_{i}(b)-f_{i}(c)\right)^{2}+2\left(f_{i}(b)-f_{i}(c)\right)\left(f_{i}(c)-f_{i}(a)\right)}{2 L} \\
& -\frac{\left(f_{i}(c)-f_{i}(a)^{2}\right.}{2 L} \\
= & L \frac{(c-a)^{2}}{2}-\frac{\left(f_{i}(c)-f_{i}(a)\right)^{2}}{2 L} \\
& +L \frac{(b-c)^{2}}{2}-\frac{\left(f_{i}(b)-f_{i}(c)\right)^{2}}{2 L} \\
& +L(b-c)(c-a)-\frac{1}{L}\left(f_{i}(b)-f_{i}(c)\right)\left(f_{i}(c)-f_{i}(a)\right) \\
= & \operatorname{Vol}\left(\Omega_{i}^{k}\left(j_{\alpha}^{k}\right)\right)+\operatorname{Vol}\left(\Omega_{i}^{k}\left(j_{\beta}^{k}\right)\right) \\
& +L(b-c)(c-a)-\frac{1}{L}\left(f_{i}(b)-f_{i}(c)\right)\left(f_{i}(c)-f_{i}(a)\right) .
\end{aligned}
$$

Thus, the definition

$$
\Lambda^{k}:=L(b-c)(c-a)-\frac{1}{L}\left(f_{i}(b)-f_{i}(c)\right)\left(f_{i}(c)-f_{i}(a)\right)
$$

is given by the statement of the lemma. It remains to show $\Lambda^{k}>\Lambda>0$.

First, we assume that $\left(f_{i}(b)-f_{i}(c)\right)\left(f_{i}(c)-f_{i}(a)\right) \leq 0$. Then (4.21) is applied to formulate

$$
\begin{aligned}
\Lambda^{k} \geq L(b-c)(c-a) \stackrel{(4.20)}{\geq} \frac{L}{16} d^{2} \stackrel{(4.19)}{=} \frac{L}{16}(b-a)^{2} \\
=\frac{L^{2}(b-a)^{2}}{16 L} \stackrel{(4.18)}{>} \frac{\varepsilon^{2}}{16 L}=: \Lambda>0 .
\end{aligned}
$$


Finally, (4.15) is proven for the first case.

Second, we assume that $\left(f_{i}(b)-f_{i}(c)\right)\left(f_{i}(c)-f_{i}(a)\right)>0$. In this case, the two factors $f_{i}(b)-f_{i}(c)$ and $f_{i}(c)-f_{i}(a)$ have equal sign and are not zero. This means that either $f_{i}(a)<f_{i}(c)<f_{i}(b)$ or $f_{i}(a)>f_{i}(c)>f_{i}(b)$ hold. We assume that the first is the case, i.e., $f_{i}(a)<f_{i}(c)<f_{i}(b)$; see Figure 4.10. The other case can be treated analogously. The inequalities

$$
f_{i}(b)-f_{i}(c) \leq L(b-c) \quad \text { and } \quad f_{i}(c)-f_{i}(a) \leq L(c-a)
$$

hold because of Lipschitz continuity. From (4.17), we can follow that at least one of the inequalities

$$
f_{i}(b)-f_{i}(c) \leq L(b-c)-\frac{\varepsilon}{2} \quad \text { or } \quad f_{i}(c)-f_{i}(a) \leq L(c-a)-\frac{\varepsilon}{2}
$$

is true. We assume that

$$
f_{i}(b)-f_{i}(c) \leq L(b-c)-\frac{\varepsilon}{2}
$$

holds. The other case can again be treated analogously. We can now estimate the term $\Lambda^{k}$ from (4.21). First, by Lipschitz continuity, we obtain

$$
\frac{1}{L}\left(f_{i}(c)-f_{i}(a)\right) \leq c-a
$$

Finally, we state

$$
\begin{aligned}
\Lambda^{k} & =L(b-c)(c-a)-\frac{1}{L}\left(f_{i}(b)-f_{i}(c)\right)\left(f_{i}(c)-f_{i}(a)\right) \\
& =L(b-c)(c-a)-\left(f_{i}(b)-f_{i}(c)\right) \frac{1}{L}\left(f_{i}(c)-f_{i}(a)\right) \\
& \stackrel{(4.23)}{\geq} L(b-c)(c-a)-\left(f_{i}(b)-f_{i}(c)\right)(c-a) \\
& =(c-a)\left[L(b-c)-\left(f_{i}(b)-f_{i}(c)\right)\right] .
\end{aligned}
$$

According to (4.22), the term in square brackets, i.e., the second factor, is bounded from below by $\varepsilon / 2$. Moreover, according to (4.20), the first factor is bounded from below by $d / 4$. Thus, we conclude

$$
\Lambda^{k} \geq \frac{d \varepsilon}{8} \stackrel{(4.19)}{>} \frac{(b-a) \varepsilon}{8} \stackrel{(4.18)}{>} \frac{\varepsilon^{2}}{8 L}>\frac{\varepsilon^{2}}{16 L}=\Lambda>0 .
$$

We are now ready to prove the main theorem for Algorithm 4.2. 
Theorem 4.5.5. Algorithm 4.2 terminates after a finite number of iterations with a globally optimal $\varepsilon$-feasible solution of (4.2) or with an indication that (4.2) is infeasible.

Proof. As for the proof of Theorem 4.5.5, we assume that the algorithm does not terminate after a finite number of iterations. Then there exists a sequence $\left(\widetilde{x}^{k}\right)_{k}$ of master problem's solutions such that all $(\mathrm{M}(k))$ are feasible and such that there exists at least one $i \in[\sigma]$ with $\left|f_{i}\left(\widetilde{x}_{i_{1}}^{k}\right)-\widetilde{x}_{i_{2}}^{k}\right|>\varepsilon$ for all $k$. As there are only finitely many possible values in $[\sigma]$, there is at least one $i \in[\sigma]$ for which $\left|f_{i}\left(\widetilde{x}_{i_{1}}^{k}\right)-\left(\widetilde{x}_{i_{2}}^{k}\right)\right|>\varepsilon$ infinitely often. We denote the corresponding subsequence by $\tau$. By Lemma 4.5.4, we know that $\operatorname{Vol}\left(\Omega_{i}^{\tau}\right)=\operatorname{Vol}\left(\Omega_{i}^{\tau-1}\right)-\Lambda^{\tau}$ with $\Lambda^{\tau}>\Lambda$ for some $\Lambda>0$. As a consequence for the selected $i$,

$$
0 \leq \operatorname{Vol}\left(\Omega_{i}^{k}\right) \rightarrow-\infty
$$

holds. This contradicts our assumption that there are infinitely many iterations.

\subsection{The Case of Lipschitz Continuous Functions and Inexact Function Evaluations}

In this section, we assume that Assumption 4.2.3 holds. We concentrate on the small differences compared with Assumption 4.2.2 in Section 4.5. Again, Section 4.6.1 describes the different parts of the algorithm. In Section 4.6.2, we then prove that the algorithm correctly terminates with a globally optimal $\varepsilon$-feasible point of Problem (4.2) or the indication of infeasibility. We remark that significant parts of this section can be found in our article Schmidt et al. [141]. Again, note that the following descriptions amplify the short and concise presentations of the article.

\subsubsection{Derivation of the Algorithm}

Master Problem The description of the master problem is similar to Section 4.5.1. Once more, we assume that there are values $x_{i_{1}}^{k, j} \in \mathbb{R}$ with

$$
x_{i_{1}}^{k, 0}<x_{i_{1}}^{k, 1}<\cdots<x_{i_{1}}^{k, r_{i}^{k}}
$$




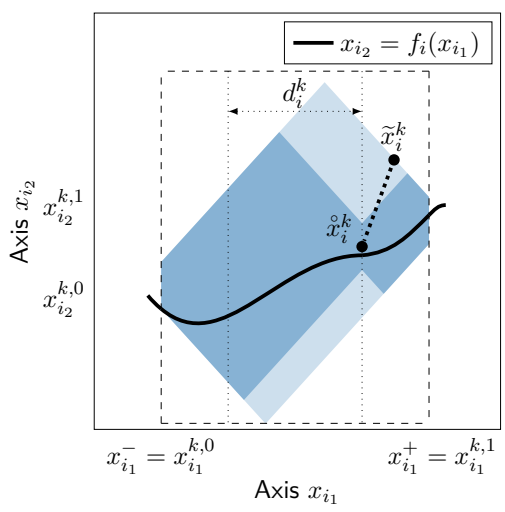

(a) Subproblem $\left(\mathrm{S}^{\prime \prime}(i, k)\right)$ for an $i \in[\sigma]$.

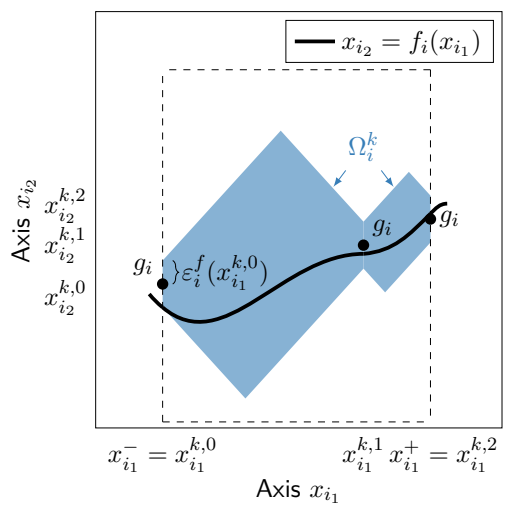

(b) Set $\Omega_{i}^{k}$ of master problem $(\mathrm{M}(k))$ for an $i \in[\sigma]$.

Figure 4.11: Setting for subproblem and master problem for Algorithm 4.3.

and this time $g_{i}\left(x_{i_{1}}^{k, j}\right)=: x_{i_{2}}^{k, j} \in \mathbb{R}$ for $j \in\left[r_{i}^{k}\right]$ to abbreviate

$$
\begin{aligned}
\mathcal{L}_{i}^{k} & :=\left\{x_{i_{1}}^{-}=: x_{i_{1}}^{k, 0}, x_{i_{1}}^{k, 1}, \ldots, x_{i_{1}}^{k, r_{i}^{k}-1}, x_{i_{1}}^{k, r_{i}^{k}}:=x_{i_{1}}^{+}\right\}, \\
\mathcal{C}_{i}^{k} & :=\left\{g_{i}\left(x_{i_{1}}^{-}\right)=: x_{i_{2}}^{k, 0}, x_{i_{2}}^{k, 1}, \ldots, x_{i_{2}}^{k, r_{i}^{k}-1}, x_{i_{2}}^{k, r_{i}^{k}}:=g_{i}\left(x_{i_{1}}^{+}\right)\right\} .
\end{aligned}
$$

The difference is that we are dealing with $g$ instead of $f$. We adjust the definition of $\Omega_{i}^{k}(j)$ for all $j \in\left[r_{i}^{k}-1\right]$ such that the master problem $(\mathrm{M}(k))$ is still a relaxation of Problem (4.2) and that it is an MIP. With the inexact evaluations $g_{i}$ of $f_{i}$, we have to modify (4.13). We define the sets $\Omega_{i}^{k}(j)$ for $j \in\left[r_{i}^{k}-1\right]$ as

$$
\begin{aligned}
\Omega_{i}^{k}(j):=\{ & \left(x_{i_{1}}, x_{i_{2}}\right) \in \mathbb{R}^{2}: x_{i_{1}}^{k, j} \leq x_{i_{1}} \leq x_{i_{1}}^{k, j+1}, \\
& x_{i_{2}} \leq x_{i_{2}}^{k, j}+\varepsilon_{i}^{f}\left(x_{i_{1}}^{k, j}\right)+L_{i}\left(x_{i_{1}}-x_{i_{1}}^{k, j}\right), \\
& x_{i_{2}} \geq x_{i_{2}}^{k, j}-\varepsilon_{i}^{f}\left(x_{i_{1}}^{k, j}\right)-L_{i}\left(x_{i_{1}}-x_{i_{1}}^{k, j}\right), \\
& x_{i_{2}} \leq x_{i_{2}}^{k, j+1}+\varepsilon_{i}^{f}\left(x_{i_{1}}^{k, j+1}\right)+L_{i}\left(x_{i_{1}}^{k, j+1}-x_{i_{1}}\right), \\
& \left.x_{i_{2}} \geq x_{i_{2}}^{k, j+1}-\varepsilon_{i}^{f}\left(x_{i_{1}}^{k, j+1}\right)-L_{i}\left(x_{i_{1}}^{k, j+1}-x_{i_{1}}\right)\right\}
\end{aligned}
$$

and obtain $\Omega_{i}^{k}$ for all $i \in[\sigma]$ together with (4.6); see Figure 4.11b for an illustration. In this case, every single $\Omega_{i}^{k}(j)$ for all $j \in\left[r_{i}^{k}-1\right]$ is a hexagon derived from the quadrilaterals before. We take over Proposition 4.4.1 (or Proposition 4.5.1) directly by construction. 
Proposition 4.6.1. For iteration $k$, we define $\Omega_{i}^{k}$ with (4.6) and (4.24) for all $i \in[\sigma]$. Then

$$
\Omega_{i}^{k} \supseteq \operatorname{gr}\left(f_{i}\right) \cap\left[x_{i}^{-}, x_{i}^{+}\right]
$$

holds. Thus, the kth master problem $(\mathrm{M}(k))$ is a relaxation of (4.2).

We derive a corollary from the proof of Lemma 4.5.2.

Corollary 4.6.2. For iteration $k$, we define $\Omega_{i}^{k}$ with (4.6) and (4.24) for all $i \in[\sigma]$. The master problem $(\mathrm{M}(k))$ is an MIP.

Proof. This follows directly from the proof of Lemma 4.5.2 and with the adjusted MIP-formulation

$$
\begin{aligned}
& -M\left(1-\omega_{i}^{k, j}\right)+x_{i_{1}}^{k, j} \leq x_{i_{1}} \leq x_{i_{1}}^{k, j+1}+M\left(1-\omega_{i}^{k, j}\right) \\
& \text { for all } j \in\left[r_{i}^{k}-1\right] \text {, } \\
& x_{i_{2}} \leq x_{i_{2}}^{k, j}+\varepsilon_{i}^{f}\left(x_{i_{1}}^{k, j}\right)+L_{i}\left(x_{i_{1}}-x_{i_{1}}^{k, j}\right)+M\left(1-\omega_{i}^{k, j}\right) \\
& \text { for all } j \in\left[r_{i}^{k}-1\right] \text {, } \\
& x_{i_{2}} \geq x_{i_{2}}^{k, j}-\varepsilon_{i}^{f}\left(x_{i_{1}}^{k, j}\right)-L_{i}\left(x_{i_{1}}-x_{i_{1}}^{k, j}\right)-M\left(1-\omega_{i}^{k, j}\right) \\
& \text { for all } j \in\left[r_{i}^{k}-1\right] \text {, } \\
& x_{i_{2}} \leq x_{i_{2}}^{k, j+1}+\varepsilon_{i}^{f}\left(x_{i_{1}}^{k, j+1}\right)+L_{i}\left(x_{i_{1}}^{k, j+1}-x_{i_{1}}\right)+M\left(1-\omega_{i}^{k, j}\right) \\
& \text { for all } j \in\left[r_{i}^{k}-1\right] \text {, } \\
& x_{i_{2}} \geq x_{i_{2}}^{k, j+1}-\varepsilon_{i}^{f}\left(x_{i_{1}}^{k, j+1}\right)-L_{i}\left(x_{i_{1}}^{k, j+1}-x_{i_{1}}\right)-M\left(1-\omega_{i}^{k, j}\right) \\
& \text { for all } j \in\left[r_{i}^{k}-1\right] \text {, } \\
& \sum_{j \in\left[r_{i}^{k}-1\right]} \omega_{i}^{k, j}=1, \\
& \omega_{i}^{k, j} \in\{0,1\} \quad \text { for all } j \in\left[r_{i}^{k}-1\right],
\end{aligned}
$$

for all $\Omega_{i}^{k}$ with $i \in[\sigma]$ in the $k$ th master problem.

The general situation for the master problem $(\mathrm{M}(k))$ as an MIP is the same as before in Section 4.4 and Section 4.5.

Subproblem The description of the subproblem is the same as in Section 4.5.1. We only have to replace $f$ by $g$ to obtain

$$
\begin{array}{ll}
\min _{x_{i}} & \left\|x_{i}-\widetilde{x}_{i}^{k}\right\|_{2}^{2} \\
\text { s.t. } & x_{i} \in \operatorname{gr}\left(g_{i}\right), \\
& x_{i} \in \stackrel{\circ}{\Omega}_{i}^{k}\left(j_{i}^{k}\right),
\end{array}
$$




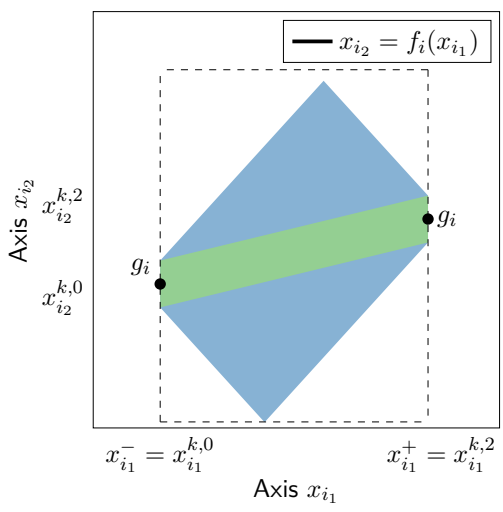

(a) 1st iteration.

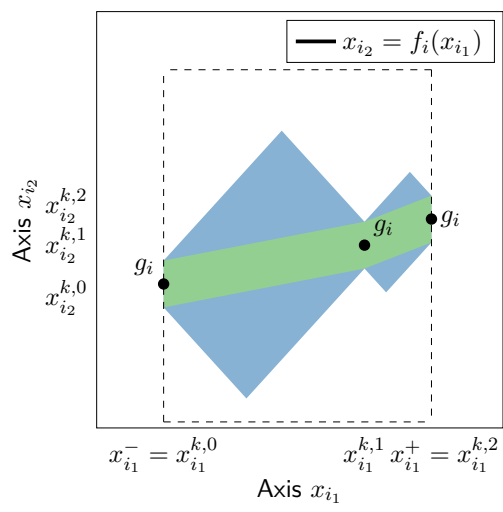

(b) 2nd iteration.

Figure 4.12: Illustration of Example 4.12.

where $j_{i}^{k}$ denotes the hexagon of the corresponding master problem's solution $\widetilde{x}_{i}^{k}$; see Figure 4.11a for an illustration. Again, note that Figure 4.11b shows the master problem with the result of the subproblem in Figure 4.11a. We remark that $\stackrel{\circ}{x}_{i_{2}}^{k}=g_{i}\left(\stackrel{\circ}{x}_{i_{1}}^{k}\right)$ but, in general, $\stackrel{ }{x}_{i_{2}}^{k} \neq f_{i}\left(\stackrel{\circ}{x}_{i_{1}}^{k}\right)$.

Decomposition Algorithm We are now able to formally state the decomposition algorithm for solving Problem (4.2) in case of Assumption 4.2.3; see Algorithm 4.3. Compared to Algorithm 4.2, we have to replace the exact function evaluation $f_{i}\left(x_{i_{1}}\right)$ by its approximation $g_{i}\left(x_{i_{1}}\right)$ in Line 1,8 and 12. Finally, the $\varepsilon$-checks " $\left|f_{i}\left(x_{i_{1}}^{k}\right)-x_{i_{2}}^{k}\right| \leq \varepsilon$ " and " $\left|f_{i}\left(x_{i_{1}}^{k}\right)-x_{i_{2}}^{k}\right|>\varepsilon$ " in the lines 8 and 12 have to be replaced by " $\left|g_{i}\left(\widetilde{x}_{i_{1}}^{k}\right)-\widetilde{x}_{i_{2}}^{k}\right| \leq \varepsilon-\varepsilon_{i}^{f}$ " and " $\left|g_{i}\left(\widetilde{x}_{i_{1}}^{k}\right)-\widetilde{x}_{i_{2}}^{k}\right|>\varepsilon-\varepsilon_{i}^{f}$ ", respectively. The value $\varepsilon_{i}^{f}$ is given by (4.4).

\subsubsection{Convergence Results}

The analysis of Algorithm 4.3 compared to Algorithm 4.2 is nearly identical. It is intuitively correct that there is a volume reduction for the next iteration again. Nevertheless, we consider the following example:

Example 4.6.3. We have Figure 4.12a for the 1st iteration and Figure 4.12b for the 2nd iteration. We observe volume reduction again. Nevertheless, there is always a green stripe determined by $\varepsilon_{i}^{f}$ that is never removed completely, i.e., it is not true that the volume reduction tends towards zero as in the proof of Theorem 4.5.5. 
Algorithm 4.3 Decomposition Method in Case of Assumption 4.2.3

Input: Problem (4.2) and $\varepsilon>0$.

Output: If there is an $\varepsilon$-feasible point for Problem (4.2), the algorithm returns a globally optimal $\varepsilon$-feasible solution $\widetilde{x}$. Otherwise, it returns an indication of infeasibility.

1: Set $k \leftarrow 1$ and define $\mathcal{L}_{i}^{k}:=\left\{x_{i_{1}}^{-}, x_{i_{1}}^{+}\right\}, \mathcal{C}_{i}^{k}:=\left\{g_{i}\left(x_{i_{1}}^{-}\right), g_{i}\left(x_{i_{1}}^{+}\right)\right\}$for all $i \in[\sigma]$.

2: while true do

3: $\quad$ Solve master problem $(\mathrm{M}(k))$ with $\mathcal{L}_{i}^{k}$ and $\mathcal{C}_{i}^{k}$ to global optimality where $\Omega_{i}^{k}$ is defined by (4.25) for all $i \in[\sigma]$.

4: if master problem $(\mathrm{M}(k))$ is infeasible then

5: return "Problem (4.2) is infeasible".

6: $\quad$ end if

7: $\quad$ Denote the solution of master problem $(\mathrm{M}(k))$ by $\widetilde{x}^{k}$.

8: $\quad$ if $\left|g_{i}\left(\widetilde{x}_{i_{1}}^{k}\right)-\widetilde{x}_{i_{2}}^{k}\right| \leq \varepsilon-\varepsilon_{i}^{f}$ for all $i \in[\sigma]$ then

9: $\quad$ return globally optimal $\varepsilon$-feasible solution $\widetilde{x}^{k}$.

10: end if

11: $\quad$ for $i \in[\sigma]$ do

12: $\quad$ if $\left|g_{i}\left(\widetilde{x}_{i_{1}}^{k}\right)-\widetilde{x}_{i_{2}}^{k}\right|>\varepsilon-\varepsilon_{i}^{f}$ then

13: $\quad$ Determine the polytopes $j_{i}^{k} \in\left[r_{k}^{i}-1\right]$ out of the master problem's solution $\widetilde{x}_{i}^{k}$.

14: $\quad$ Solve subproblems $\left(\mathrm{S}^{\prime \prime}(i, k)\right)$ for yielding solutions $\stackrel{\circ}{x}_{i}^{k}$ and set $\mathcal{L}_{i}^{k+1} \leftarrow \mathcal{L}_{i}^{k} \cup\left\{\dot{o}_{i_{1}}^{k}\right\}$ and $\mathcal{C}_{i}^{k+1} \leftarrow \mathcal{C}_{i}^{k} \cup\left\{\stackrel{o}{x}_{i_{2}}^{k}\right\}$.

15: $\quad$ else

16: $\quad$ Set $\mathcal{L}_{i}^{k+1} \leftarrow \mathcal{L}_{i}^{k}$ and $\mathcal{C}_{i}^{k+1} \leftarrow \mathcal{C}_{i}^{k}$.

17: $\quad$ end if

18: end for

19: $\quad$ Increase $k \leftarrow k+1$.

20: end while

We remark that Example 4.6.3 has to be taken into account. Note that if the green stripe is the only feasible area left, we could guarantee an $\varepsilon$-feasible solution if $\varepsilon$ is chosen large enough compared to $\varepsilon_{i}^{f}$. Consequently, Algorithm 4.3 would terminate. Thus, a recipe to prove finite termination would be an adapted proof of Theorem 4.5.5 making clear that the case of Example 4.6.3 is not a problem.

Nevertheless, we want to present a slightly different proof that relies on the reduction of the intervals. As soon as the intervals are small enough, 
we can guarantee an $\varepsilon$-feasible solution as long as $\varepsilon$ is chosen large enough compared to $\varepsilon_{i}^{f}$, i.e., as long as (4.5) inside of Assumption 4.2.3 holds. We remark that this kind of proof could be used for Theorem 4.5.5 as well.

Theorem 4.6.4. Algorithm 4.3 terminates after a finite number of iterations with a globally optimal $\varepsilon$-feasible solution of (4.2) or with an indication that (4.2) is infeasible.

Proof. As for the proof of Theorem 4.4.7 and Theorem 4.5.5, we assume that the algorithm does not terminate after a finite number of iterations. Then there exists a sequence $\left(\widetilde{x}^{k}\right)_{k}$ of master problem's solutions such that all $(\mathrm{M}(k))$ are feasible and such that there exists at least one $i \in[\sigma]$ with $\left|g_{i}\left(\widetilde{x}_{i_{1}}^{k}\right)-\widetilde{x}_{i_{2}}^{k}\right|>\varepsilon-\varepsilon_{i}^{f}$ for all $k$. As there are only finitely many possible values in $[\sigma]$, there is at least one $i \in[\sigma]$ for which $\left|g_{i}\left(\widetilde{x}_{i_{1}}^{k}\right)-\widetilde{x}_{i_{2}}^{k}\right|>\varepsilon-\varepsilon_{i}^{f}$ infinitely many times. We denote the corresponding subsequence by $\tau$.

In Line 14, a refined interval is split into two new subintervals for the next iteration. By construction, each of these subintervals has a length of at most $3 / 4$ of the original interval. Thus, the length of the intervals constantly decrease towards zero.

Due to Lipschitz continuity, the maximal vertical difference on an interval $\left(x_{i_{1}}^{\tau, j}, x_{i_{1}}^{\tau, j+1}\right)$ is bounded by

$$
2 \varepsilon_{i}^{f}+L_{i}\left(x_{i_{1}}^{\tau, j+1}-x_{i_{1}}^{\tau, j}\right)=2 \varepsilon_{i}^{f}+L_{i} d_{i}^{\tau, j} .
$$

We show that for sufficiently large $\tau$, we get

$$
d_{i}^{\tau, j} \leq \frac{\varepsilon-2 \varepsilon_{i}^{f}}{L_{i}}>0
$$

We note that the last inequality holds because of (4.5) and $L_{i}>0$. As the length of the intervals constantly decrease towards zero, (4.27) is reached eventually. Finally, we use the maximal vertical difference from (4.26) together with (4.27). For a sufficiently large $\tau$, we state

$$
\left|f_{i}\left(\widetilde{x}_{i_{1}}^{\tau}\right)-\widetilde{x}_{i_{2}}^{\tau}\right| \leq 2 \varepsilon_{i}^{f}+L_{i} d_{i}^{\tau, j} \leq 2 \varepsilon_{i}^{f}+L_{i}\left(\frac{\varepsilon-2 \varepsilon_{i}^{f}}{L_{i}}\right) \leq \varepsilon .
$$

Consequently, $\varepsilon$-feasibility is reached contradicting our assumption. 


\subsection{Literature Survey: Related Algorithms}

The most common way to solve the nonconvex MINLP (4.2) is to use solvers as Baron, Couenne, and Scip; see Baron [9], Couenne [35], and Maher et al. [110], respectively. These solvers expect analytic functions as input. Our framework does not provide these functions and restricts the knowledge about the nonlinearities; see Assumptions 4.2.1-4.2.3. Nevertheless, we outline how methods that are partially used in Baron, Couenne, and Scip are related to our framework. This comprises spatial branch-and-bound methods in general, piecewise linearization techniques for Algorithm 4.1, and ideas from the field of global Lipschitz optimization without integer variables for Algorithm 4.2 and Algorithm 4.3.

Spatial branch-and-bound strongly relies on analytic functions to convexify the nonconvex set, which again prohibits a direct application to our setting. Besides from different linearization techniques, spatial branch-and-bound solves MINLPs by building up a branch-and-bound tree including the difficulties of branching decisions. As opposed to this, our algorithms build upon our guideline to rely on MIP solvers as a workhorse.

On the other hand, we discuss piecewise linearization of the nonlinearities worked out by Burlacu et al. [24], Geißler [60], and Geißler et al. [6164]. These literature references are already mentioned in Section 3.3. We want to describe the details of these references to classify Algorithm 4.1. In Burlacu et al. [24], Geißler [60], and Geißler et al. [61-64], they also use piecewise linear relaxation based on the incremental method of Markowitz and Manne [114]. As in our case, a factorization according to McCormick [118], Smith and Pantelides [150], and Tawarmalani and Sahinidis [154] is performed. The authors propose a procedure that builds up an MIP relaxation of a nonconvex MINLP a-priori. The MIP relaxation relies on error estimators whose calculation requires analytic functions and solving NLPs; see Geißler et al. [63, Proposition 4.1]. Although similar modeling techniques are used, there are various differences. First, Burlacu et al. [24], Geißler [60], and Geißler et al. [61-64] are reliant on nonlinear functions that are given in closed form to ensure the relaxation property. Second, their constructed relaxation exclusively builds upon Markowitz and Manne [114], while we use classical outer approximation by Duran and Grossmann [50] additionally. Third, they construct the relaxations a-priori in dependence of some given tolerance and not on demand as in our case. However, Burlacu et al. [24] and Geißler [60] propose an adaptive algorithm that manages this limitation. The focus of these references, especially Burlacu et al. [24] with 
their longest edge bisection, lies on refinement strategies, while we investigate relaxation strategies. Finally, Algorithm 4.1 uses $\ell_{2}-\varepsilon$-feasibility, while Burlacu et al. [24], Geißler [60], and Geißler et al. [61-64] work with $\varepsilon$-feasibility; see Definition 4.3.2 and Definition 4.3.3.

As opposed to Algorithm 4.1, where we adapt MINLP methods, Algorithm 4.2 and Algorithm 4.3 further incorporate ideas from the field of global Lipschitz optimization without integer variables. Such problems are tackled with suitable underestimators in the form of saw-tooth covers that are presented in Evtuschenko [53] and Piyavskii [131] for the univariate case and in Pintér [126-128] for higher dimensions. On top of this, extensions for branch-and-bound are introduced in Horst [86], Horst and Thoai [87], Horst and Tuy [89], Pintér [125], and Tuy and Horst [155]. For a literature survey, see the books Hansen and Jaumard [82], Horst and Tuy [88], and Pintér [130] and the references therein. An example for a software based on these concepts is the so-called Lipschitz Global Optimizer (LGO); see Pintér [129]. Moreover, the use of relaxations instead of underestimators is considered in Kvasov and Sergeyev [102]. Finally, MINLPs with strongly convex and Lipschitz continuous functions are studied in Baes et al. [6]. All these methods deal with the linearization of a function whose Lipschitz constant is known analogous to Assumption 4.2.2 and Assumption 4.2.3. We use these ideas for the definition of $(4.13) /(4.24)$ to construct an MIP relaxation of the nonlinear functions by $(4.14) /(4.25)$.

All these descriptions refer to the closely related literature. We discuss some additional, peripheral connections inside the following Remark 4.7.1.

Remark 4.7.1 (Peripheral Literature). First, the algorithmic approaches we present build on Benders' decomposition; see Benders [14] and Geoffrion [68]. Even if Benders' decomposition relies on duality theory to prove convergence, we adapt the idea of decomposing the problem into a master problem and a subproblem. Our methods alternately solve MIP master problems and nonlinear separation subproblems to iteratively refine the MIP relaxation of the nonlinearities. We note that the standard version of Generalized Benders' decomposition does not converge for nonconvex problems; see Sahinidis and Grossmann [137]. A problem that our algorithms do not suffer from.

Second, we mention mixed-integer optimal control (MIOCP) problems; see Belotti et al. [13, Chapter 7] and Sager [136]. In this context, Buchheim et al. [20] propose an approach for MIOCP with semilinear elliptic $\mathrm{PDES}$ and static integer controls. Even if $\mathrm{MIOCP}$ addresses integers distributed in time or space, which is not considered in our case, this approach 
has some similarities with our approach as it also decouples the entire problem into a master problem and a subproblem. The former addresses all mixed-integer aspects, whereas the latter addresses the differential equation and delivers outer approximation cutting planes for the next master problem. However, the focus of Buchheim et al. [20] is more on a specific type of differential equation that yields convex feasible sets, whereas our approach abstracts from specific nonlinearities and considers nonconvex feasible sets.

Third, mixed-integer dynamic optimization (MIDO) problems, where systems governed by ODEs or PDEs are optimized over a control input, pose similar problems as the nonlinearities are not given analytically; see Chachuat et al. [27] and the references therein, Fügenschuh et al. [58] and Geißler et al. [61] for examples based on linearization.

Finally, we develop algorithms for MINLP $s$ with nonlinearities whose only usable analytical property is Lipschitz continuity in Section 4.5 and Section 4.6. Thus, these algorithms fit into frameworks like parametric or bilevel optimization where complicated nondifferentiable but Lipschitz continuous functions are frequently studied; see, e.g., Dempe [45] and Dempe et al. [46].

\subsection{Further Remarks}

We make further remarks on the decomposition algorithms. Section 4.8.1 refers to algorithmic details, whereas Section 4.8.2 gives an overview on limitations and extensions.

\subsubsection{Algorithmic Details}

Remark 4.8.1 (Feasibility). If Problem (4.2) is infeasible, the master problem can still have $\ell_{2}-\varepsilon$-feasible or $\varepsilon$-feasible solutions that are then found by the algorithms. Moreover, let $x^{*}$ be the solution of Problem (4.2) and let $\widetilde{x}$ be the solution returned by Algorithms 4.1-4.3. Then $c^{\top} \widetilde{x} \leq c^{\top} x^{*}$ holds because the algorithms always consider a relaxation of the original problem (4.2). We further remark that $\widetilde{x}$ and $x^{*}$ can be arbitrarily far apart. All these statements hold for any MINLP solution approaches that work with relaxations, e.g., spatial branch-and-bound.

Remark 4.8.2 (Cutting off the Solution of the Master Problem). We show that the solution $\widetilde{x}^{k}$ of the master problem is cut off in the next iteration for Algorithm 4.1; see Theorem 4.4.6. This is not necessarily true for 
Algorithm 4.2 and Algorithm 4.3; see Figure 4.8a again. The solution $\widetilde{x}^{k}$ remains feasible for iteration $k+1$.

Remark 4.8.3 (Growth of Master Problems). All algorithms have a similar refinement strategy. If an $\ell_{2}-\varepsilon$-feasible or $\varepsilon$-feasible solution is not obtained, refinements are necessary. The amount of refinements is bounded from above by the amount of nonlinear functions. Thus, it is immediately clear that the growth of the master problem per iteration in terms of variables and constraints is $\mathcal{O}(\sigma)$.

Remark 4.8.4 (Solving the Subproblems). Algorithms 4.1-4.3 solve various NLP s to global optimality. Nevertheless, the arguments ensuring a finite number of iterations for Algorithm 4.2 and Algorithm 4.3, that is volume reduction and interval reduction, clearly hold for every feasible point that is returned by the subproblem. We exploit that for our numerical results in Section 4.9 and use a global NLP solver for Algorithm 4.1 and a local NLP solver for Algorithm 4.2 and Algorithm 4.3.

Remark 4.8.5 (Size of the Subproblems). At the beginning of Section 4.3, we state that we want to obtain small and tractable NLPs. In point of fact, subproblems $(\mathrm{S}(i, k))$ and subproblems $\left(\mathrm{S}^{\prime}(i, k)\right)$ are only two-dimensional problems.

Remark 4.8.6 (Termination after a Finite Number of Iterations). We prove that Algorithms 4.1-4.3 terminate after a finite number of iterations. The proof of Theorem 4.4.7 for Algorithm 4.1 relies on cutting off the already visited solution $\widetilde{x}^{k}$ for all subsequent iterations. In contrast to many other global solution approaches for MINLPs, the proofs of Theorem 4.5.5 and Theorem 4.6.4 for Algorithm 4.2 and Algorithm 4.3, respectively, do not rely on that property. It is not true anyway; see Remark 4.8.2. We use volume reduction in Theorem 4.5.5, whereas we use interval reduction in Theorem 4.6.4.

Remark 4.8.7 (Number of Iterations). It is possible to give worst-case iteration bounds based on the geometrical ideas of the proofs of Algorithms 4.1-4.3. The easiest case is the volume reduction for Algorithm 4.2 where the quotient of total volume and removed volume defines a maximum number of iterations.

Note that pathological examples that lead to large worst-case estimations for the required number of iterations might exist. See Figure 4.13a for Algorithm 4.1, where the area that is cut off in every iteration is small and the subsequent master problem solutions are close to each other. On the 


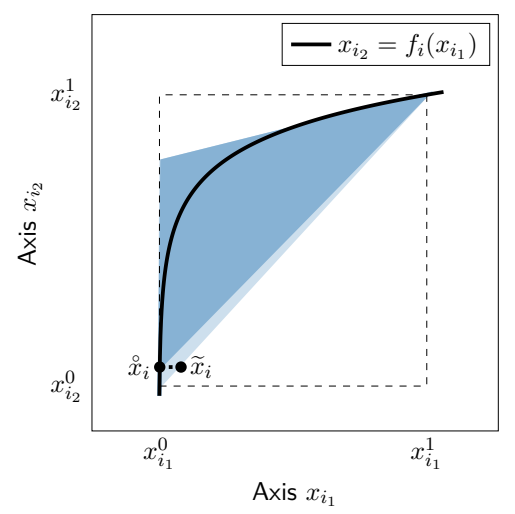

(a) Example for Algorithm 4.1.

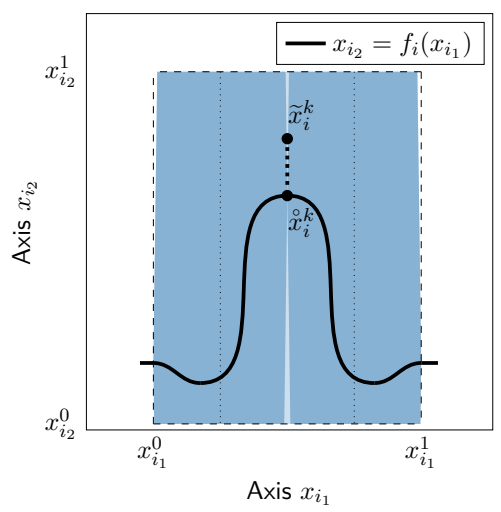

(b) Example for Algorithm 4.2.

Figure 4.13: Pathological examples with high number of iterations.

other hand, Figure 4.13b shows an example for Algorithm 4.2, where the Lipschitz constant is so large that the area that is cut off is small again. An example for Algorithm 4.3 can be formulated analogously.

\subsubsection{Limitations and Extensions}

Remark 4.8.8 (Higher Dimensions). Our working basis is a factorization of the nonlinear functions, which is state-of-the-art for nonconvex MINLP s; see McCormick [118], Smith and Pantelides [150], and Tawarmalani and Sahinidis [154]. Thus, we solve the factorized problem (4.2).

Nevertheless, we want to discuss the situation for higher dimensional functions and describe the difficulties. We consider the function $x_{i_{3}}=5\left(\log \left(x_{i_{1}}\right)+\log \left(x_{i_{2}}\right)\right)$ for Algorithm 4.1. For a linearization, we need a triangulation of the $\left(x_{i_{1}}, x_{i_{2}}\right)$-plane. This triangulation is naturally given with two triangles; see Figure 4.14 a for an illustration. We can construct an MIP relaxation with outer approximation cuts and piecewise linear underestimators analogously. The former are not included in Figure 4.14 a for the sake of simplicity. The latter are included and can be modeled with an enhancement of Markowitz and Manne [114] that is properly described in Geißler [60]. Moreover, the master problem's solution $\widetilde{x}_{i}^{k}$, the subproblem's solution $\dot{x}_{i}^{k}$, and their position on the $\left(x_{i_{1}}, x_{i_{2}}\right)$-plane are visualized. Various problems occur now.

First, Lemma 4.4.4 (b) states that the solution of the master problem and its corresponding subproblem's solution lie between the same linearization 


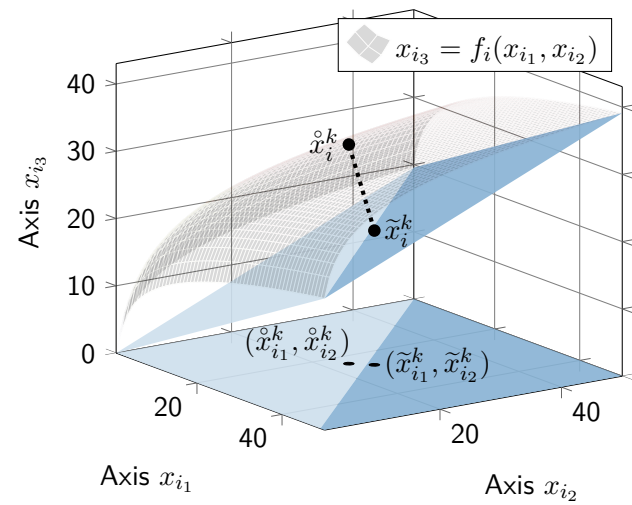

(a) Iteration $k$.

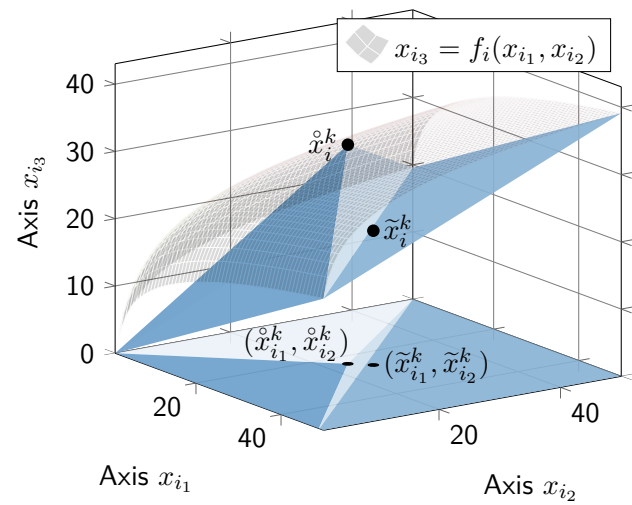

(b) Iteration $k+1$.

Figure 4.14: Algorithm 4.1 with additional dimension in $(4.2 \mathrm{c}): x_{i_{3}}=5\left(\log \left(x_{i_{1}}\right)+\log \left(x_{i_{2}}\right)\right)$.

points for one-dimensional functions. With an additional dimension, this is not true anymore; see $\left(x_{i_{1}}, x_{i_{2}}\right)$-plane in Figure $4.14 a$ and how we switch the triangle.

Second, the master problem's solution $\widetilde{x}_{i}^{k}$ is still feasible for iteration $k+1$ what might be disadvantageous to prove finite termination; see Figure $4.14 \mathrm{~b}$

Finally, the refinement process of how to do the triangulation is an open question now. The triangulation in Figure $4.14 b$ is the most naïve approach to obtain a new triangulation and it might produce numerical instabilities due to thin triangles. We can prevent that by using a Delaunay triangulation, which is commonly used in the literature. Recently, Burlacu et al. [24] 
present a longest edge bisection approach and show convergence as well as promising results for gas network problems.

Remark 4.8.9 (Possible Extensions I-Higher Dimensional Functions). If higher dimensional functions are involved, we apply factorization to obtain one-dimensional functions. Unfortunately, the factorization procedure is inconvenient if analytic functions do no exist as it is the case in our framework. Based on the algorithms and proofs we present in Chapter 4, we are developing an enhancement that overcomes that problem:

[142] M. Schmidt, M. Sirvent, and W. Wollner. "Simulation-Based MINLPs with Lipschitz Nonlinearities." Working paper. 2017.

The content of this working paper is not part of this thesis.

Thus, the presented results of this chapter develop algorithms especially for one-dimensional nonlinear functions. Moreover, and even more important, it is the basis for more general problems constrained by differential equations or models that rely on black-box simulation runs. Again, we refer to our working paper Schmidt et al. [142].

Remark 4.8.10 (Possible Extensions II-Extension of the Assumptions). Additionally to our assumptions in Section 4.2, there are obvious extensions. We can assume a case in which we have Lipschitz continuous functions but can only obtain a local Lipschitz constant, e.g., by evaluation of derivatives. Another extension is the case where the local Lipschitz constant can only be obtained approximately, e.g., due to errors in the calculation of derivatives. In both cases, the difficulties arise from Lipschitz constants that are not necessarily globally valid. This hampers the creation of a master problem $(\mathrm{M}(k))$ that is a relaxation of Problem (4.2). We propose additional algorithms to overcome these problems in our article Schmidt et al. [141]. These additional algorithms have been elaborated by Prof. Dr. Martin Schmidt and Prof. Dr. Winnifried Wollner.

\subsection{Application to Stationary Gas Transport Optimization}

Algorithms 4.1-4.3 are developed for Problem (4.2). We apply these algorithms for stationary gas transport optimization.

Remark 4.9.1 (Stationary Gas Transport Optimization). For the stationary case, the gas model (3.27) exists with only one time point, i.e., we omit all time indices in Section 4.9. 
We define an objective function for stationary gas transport optimization in Section 4.9.1. Afterward, Section 4.9.2 derives a suitable stationary pipe model. Finally, we present promising numerical results for all algorithms in Section 4.9.3.

\subsubsection{Objective Function}

For stationary gas transport optimization, the question on the objective function is closely related to the problem we want to solve. We are interested in the nomination validation problem. Numerous studies investigate the nomination validation problem and we refer to Fügenschuh et al. [57] and Ríos-Mercado and Borraz-Sánchez [133] as well as the references therein.

Customers of gas transport companies supply or discharge gas at certain points of the network. These customers announce - or nominate- the concrete amount of gas for the next day and such an announcement is called nomination. As all of these nominations are fixed, we fix the mass flow variables $q_{u}$ from (3.2a) to $\hat{q}_{u}$. For additional information about regulatory rules for gas markets in Germany, we refer to Gotzes et al. [72].

A nomination is always balanced, i.e., the quantity of announced gas to supply equals the quantity of announced gas to discharge. Mathematically speaking,

$$
\sum_{u \in V} \hat{q}_{u}=0
$$

holds for every nomination. The aim is to find a configuration for all controllable elements that satisfies all customers in regard to their nominated amount of gas.

Finally, in such a setting, minimizing the total required power

$$
\sum_{a \in A_{\mathrm{cm}}} P_{a}
$$

of the compressor machines is the most typical objective. Note that this is a linear objective function as required in (4.2a).

\subsubsection{Stationary Pipe Model}

We show how the constraints of Problem (4.2) correspond to the gas model (3.27). The mixed-integer linear part (4.2b) is directly represented by $(3.27 \mathrm{a})-(3.27 \mathrm{~g})$. The nonlinear part $(4.2 \mathrm{c})$ is more complicated. It is determined by the pipe model $(3.27 \mathrm{~h})$.

We explain the derivation of our stationary pipe model and show relevant properties afterward. 


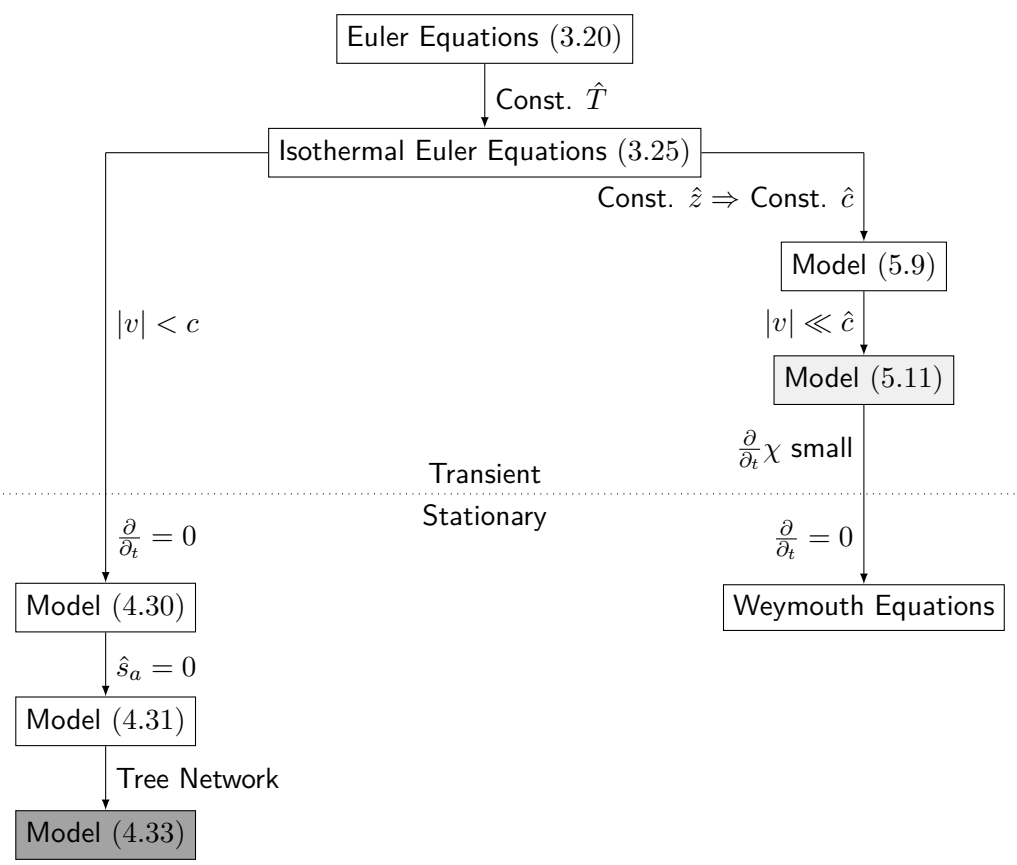

Figure 4.15: Classification of our stationary pipe model (4.33) in dark gray compared to our transient pipe model (5.11) of Chapter 5 in light gray.

\section{Derivation}

We start with pipe model $(3.27 \mathrm{~h})$ and simplify. Note that it consists of the isothermal Euler equations (3.25) and the definition of the compressibility factor $z$ in (3.23). First, we obtain the speed of sound $c=c(z) \in \mathbb{R}$ calculated by

$$
c(z)=\hat{R}_{\mathrm{s}} \hat{T} z .
$$

The compressibility factor $z$ depends on the pressure $p$; see (3.23) again. Moreover, the pressure $p$ depends on the spatial coordinate $x$. In this way, the speed of sound $c$ depends on the spatial coordinate as well. We assume that $|v|<c$, i.e., $|v|<\hat{R}_{\mathrm{s}} \hat{T} z$; see Figure 4.15. This is an assumption that holds for gas transportation networks in general. 
Second, the time derivatives $\partial / \partial_{t}$ equal zero as we are in the stationary case; see Remark 4.9.1. Hence, (3.25) simplifies to

$$
\begin{aligned}
\hat{R}_{\mathrm{s}} \hat{T} z(p) \partial_{x} \chi & =0, \\
\partial_{x}\left(p+\frac{\hat{R}_{\mathrm{s}} \hat{T} z(p)}{p} \chi^{2}\right) & =-\frac{\hat{\theta}_{a} \hat{R}_{\mathrm{s}} \hat{T} z(p)}{2} \frac{\chi|\chi|}{p}-\frac{\hat{g} \hat{s}_{a}}{\hat{R}_{\mathrm{s}} \hat{T} z(p)} p .
\end{aligned}
$$

Third, we restrict ourselves to horizontal pipes, i.e., $\hat{s}_{a}=0$. Thus, we arrive at a pipe model with

$$
\begin{aligned}
\hat{R}_{\mathrm{s}} \hat{T} z(p) \partial_{x} \chi & =0, \\
\partial_{x}\left(p+\frac{\hat{R}_{\mathrm{s}} \hat{T} z(p)}{p} \chi^{2}\right) & =-\frac{\hat{\theta}_{a} \hat{R}_{\mathrm{s}} \hat{T} z(p)}{2} \frac{\chi|\chi|}{p} .
\end{aligned}
$$

The continuity equation (4.31a) states constant mass flux for every pipe. This justifies the choice for a single mass flux variable

$$
\chi_{a}=\chi_{a, u}=\chi_{a, w} \quad \text { for all } a=(u, w) \in A_{\mathrm{pi}}
$$

and, consequently, a single mass flow variable

$$
q_{a}=q_{a, u}=q_{a, w} \quad \text { for all } a=(u, w) \in A_{\mathrm{pi}}
$$

with

$$
\chi_{a}=\frac{q_{a}}{\hat{A}_{a}} \quad \text { for all } a \in A_{\mathrm{pi}}
$$

analogous to (3.26). Note that single mass flow variables are chosen for valves, control valves, and compressor machines as well; see (3.3).

Finally, we assume transport networks to be trees - a case that is frequently discussed in the literature; see Ríos-Mercado and BorrazSánchez [133] for a recent survey. In this case, our mass flow variables $q_{a}$ are fixed to $\hat{q}_{a}$ according to mass flow conservation (3.6) and according to the fixed nominations in nomination validation problems. Accordingly, the mass flux variables $\chi_{a}$ are fixed to $\hat{\chi}_{a}$ as well; see (4.32). Eventually, we obtain the stationary pipe model

$$
\partial_{x}\left(p+\frac{\hat{R}_{\mathrm{S}} \hat{T} z(p)}{p} \hat{\chi}_{a}^{2}\right)=-\frac{\hat{\theta}_{a} \hat{R}_{\mathrm{S}} \hat{T} z(p)}{2} \frac{\hat{\chi}_{a}\left|\hat{\chi}_{a}\right|}{p} .
$$

Figure 4.15 illustrates the classification of this stationary pipe model compared to the transient pipe model used in Chapter 5. Note that similar figures to classify gas models are used in Joormann et al. [93]. The remaining balance of moments (4.33) describes the pressure loss in a pipe due to ram pressure and frictional forces with a pressure function $p=p(x) \in \mathbb{R}_{>0}$ along the pipe, i.e., $x \in\left[0, \hat{L}_{a}\right]$. 


\section{Properties}

We show how the involved variables $p_{u}$ and $p_{w}$ fit the stationary pipe model (4.33). We remark that flow variables are not relevant any more as they are fixed. Afterward, we have to clarify how this fits the constraint $x_{i_{2}}=f_{i}\left(x_{i_{1}}\right)$ in $(4.2 \mathrm{c})$ and derive the relevant properties.

The coupling of the pressure solution of (4.33) with node pressure variables $p_{u}$ and $p_{w}$ is given by

$$
p_{u}=p(0), \quad p_{w}=p\left(\hat{L}_{a}\right) \quad \text { for all } a=(u, w) \in A_{\mathrm{pi}} .
$$

The functions $f_{a}$ correspond to the relation between in- and outflow pressures of the pipes. Thus, the constraint

$$
x_{i_{2}}=f_{i}\left(x_{i_{1}}\right) \quad \text { for all } i \in[\sigma]
$$

in $(4.2 \mathrm{c})$ is represented by

$$
p_{w}=f_{a}\left(p_{u}\right) \quad \text { for all } a=(u, w) \in A_{\mathrm{pi}}
$$

in our stationary pipe model. The set $[\sigma]$ corresponds to the set $A_{\text {pi }}$.

The derivation of the functions $f_{a}$ for (4.33) and its properties are given by Gugat et al. [78] and reconsidered in our article Gugat et al. [77]. These findings have been elaborated by Prof. Dr. Martin Gugat and David Wintergerst. Therefore, we only refer to the results that are relevant in our application.

We consider $\hat{\chi}_{a}>0$. This can always be obtained by redefining the direction of the pipe if $\hat{\chi}_{a}<0$. The case $\hat{\chi}_{a}=0$ is irrelevant as it implies $p_{u}=p_{w}$ immediately. Finally, the pressure loss along pipe $a=(u, w) \in A_{\mathrm{pi}}$ for given inflow pressure $p_{u}$ derived from the stationary pipe model (4.33) reads

$$
\begin{array}{r}
f_{a}\left(p_{u}\right)=F_{a}^{-1}\left(F_{a}\left(p_{u}\right)-\frac{1}{2} \hat{R}_{\mathrm{s}} \hat{T} \hat{\chi}_{a}\left|\hat{\chi}_{a}\right| \hat{\theta}_{a} \hat{L}_{a}\right), \\
F_{a}\left(p_{u}\right)=\frac{1}{\hat{\alpha}} p_{u}+\left(\hat{\chi}_{a}^{2} \hat{R}_{\mathrm{S}} \hat{T}-\frac{1}{\hat{\alpha}^{2}}\right) \ln \left(\left|1+\hat{\alpha} p_{u}\right|\right)-\hat{\chi}_{a}^{2} \hat{R}_{\mathrm{S}} \hat{T} \ln \left(p_{u}\right) .
\end{array}
$$

See Gugat et al. [78] once more. Note that (4.34) is a solution of our stationary pipe model (4.33). We remark that $\hat{\alpha}$ is defined in (3.23). Furthermore, Gugat et al. [78] show that the use of the inverse in (4.34a) is justified because $F_{a}$ is strictly increasing in $p_{u}$ under the given assumptions with

$$
F_{a}^{\prime}\left(p_{u}\right)=\frac{p_{u}^{2}-\hat{\chi}_{a}^{2} \hat{R}_{\mathrm{s}} \hat{T}}{p_{u}\left(1+\hat{\alpha} p_{u}\right)}>0 .
$$


We remark that Gugat et al. [78] prove that functions $f_{a}$ are strictly increasing and strictly concave. We cite the central theorem without the proof.

Theorem 4.9.2 (Gugat, Wintergerst). We assume $\hat{\chi}_{a}>0$. Moreover, $f_{a}$ and $F_{a}$ are defined in (4.34) and derived from the stationary pipe model (4.33). Then

$$
\begin{array}{r}
f_{a}^{\prime}\left(p_{u}\right)=\frac{F_{a}^{\prime}\left(p_{u}\right)}{F_{a}^{\prime}\left(p_{w}\right)}>0, \\
f_{a}^{\prime \prime}\left(p_{u}\right)<0,
\end{array}
$$

hold. Thus, functions $f_{a}$ are strictly increasing and strictly concave.

Hence, Assumption 4.2.1 holds for functions $f_{a}$ according to Theorem 4.9.2. We obtain the oracle for $f_{a}$ by calculating (4.34a) with Newton's method. The Lipschitz constants $L_{i}$ for Assumption 4.2.2 and Assumption 4.2.3 are calculated by evaluating (4.35a) at $p_{u}=p_{u}^{-}$in case of $\hat{\chi}_{a}>0$. We remark that this holds because the function is strictly increasing and strictly concave.

\subsubsection{Numerical Results}

Finally, our stationary gas transport optimization problem, a nomination validation problem, reads

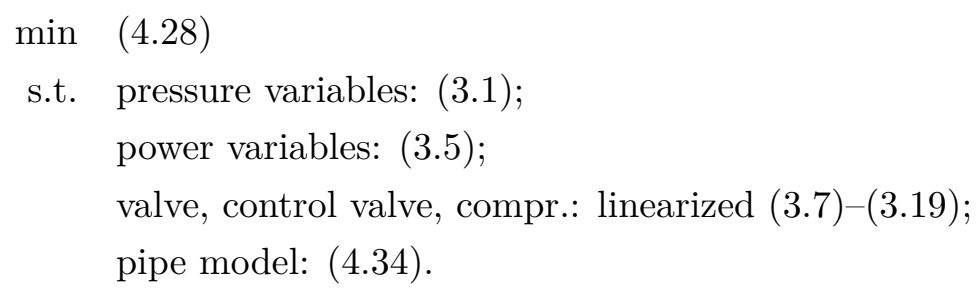

We remark that Section 4.9.2 derives a model with fixed flow. That is because we consider nomination validation problems on transport networks that are trees. Hence, all mass flow and mass flux variables can be seen as parameters that fulfill mass flow conservation and are not additionally defined in Problem (StatGas).

Remark 4.9.3 (Classification of Problem (StatGas)). Pipe model (4.34) consists of constraints that exist analytically. Hence, Problem (StatGas) is an MINLP such that standard MINLP solvers like Baron, Couenne, 
and Scip, or (piecewise) linearizations could be used to solve the whole problem; see Baron [9], Couenne [35], and Maher et al. [110], respectively. Nevertheless, these general-purpose MINLP solvers are not tailored for gas transport optimization.

In our framework, we now pretend that we are restricted to the requirements defined in Assumptions 4.2.1-4.2.3. Hence, Algorithms 4.1-4.3 are more general than it would be necessary for Problem (StatGas). On the other hand, to the best of our knowledge, almost all of the algorithms for stationary gas transport optimization, especially when including discrete decisions, deal with the Weymouth equation as pipe model; see Figure 4.15. Typically, the Weymouth equation is (piecewise) linearized. Our pipe model (4.34) omits additional simplification steps that are needed to derive the Weymouth equation; see Figure 4.15 again. In particular, we model the exact compressibility factor $z$.

Taken all together, Problem (StatGas) is an example for Problem (4.2) and we can use Algorithms 4.1-4.3. Hence, stationary gas transport optimization is a good example to show the general applicability of our algorithms.

Our real-world test instance is the Greek natural gas transport network. We first introduce the data and show promising numerical results afterward. Algorithms 4.1-4.3 iteratively solve an MIP and multiple NLP models. Our decomposition algorithms and all models are implemented using the $\mathrm{C}++$ framework LaMaTTO++; see LaMaTTO++ [103]. We solve the MIPs with Gurobi 6.5.0 using all the available 4 threads; see Gurobi [80]. The small NLPs are solved with Baron 12.3.3 for Algorithm 4.1 and with Ipopt 24.1.3 for Algorithms 4.2-4.3; see Baron [9] and Wächter and Biegler [157], respectively. Note that Baron is a global MINLP, which implies NLPs, solver, whereas Ipopt is a local NLP solver and that this is in line with our algorithmic framework; see Remark 4.8.4. The data analysis of the results was performed by Python Pandas 0.20.3 inside of Python 3.5.2; see McKinney [119]. All computations were performed on an Intel ${ }^{\odot}$ Core $^{\mathrm{TM}} \mathrm{i} 5-3360 \mathrm{M}$ CPU with 4 cores and $2.8 \mathrm{GHz}$ each and $4 \mathrm{~GB}$ RAM.

We first introduce the data and show promising numerical results for all algorithms. We remark that the results for Algorithm 4.1 are different compared to our article Gugat et al. [77] as we use a different NLP solver for the subproblems. On the other hand, the results for Algorithm 4.2 and Algorithm 4.3 are replicated from our article Schmidt et al. [141]. Finally, we close with remarks and interpretations. 


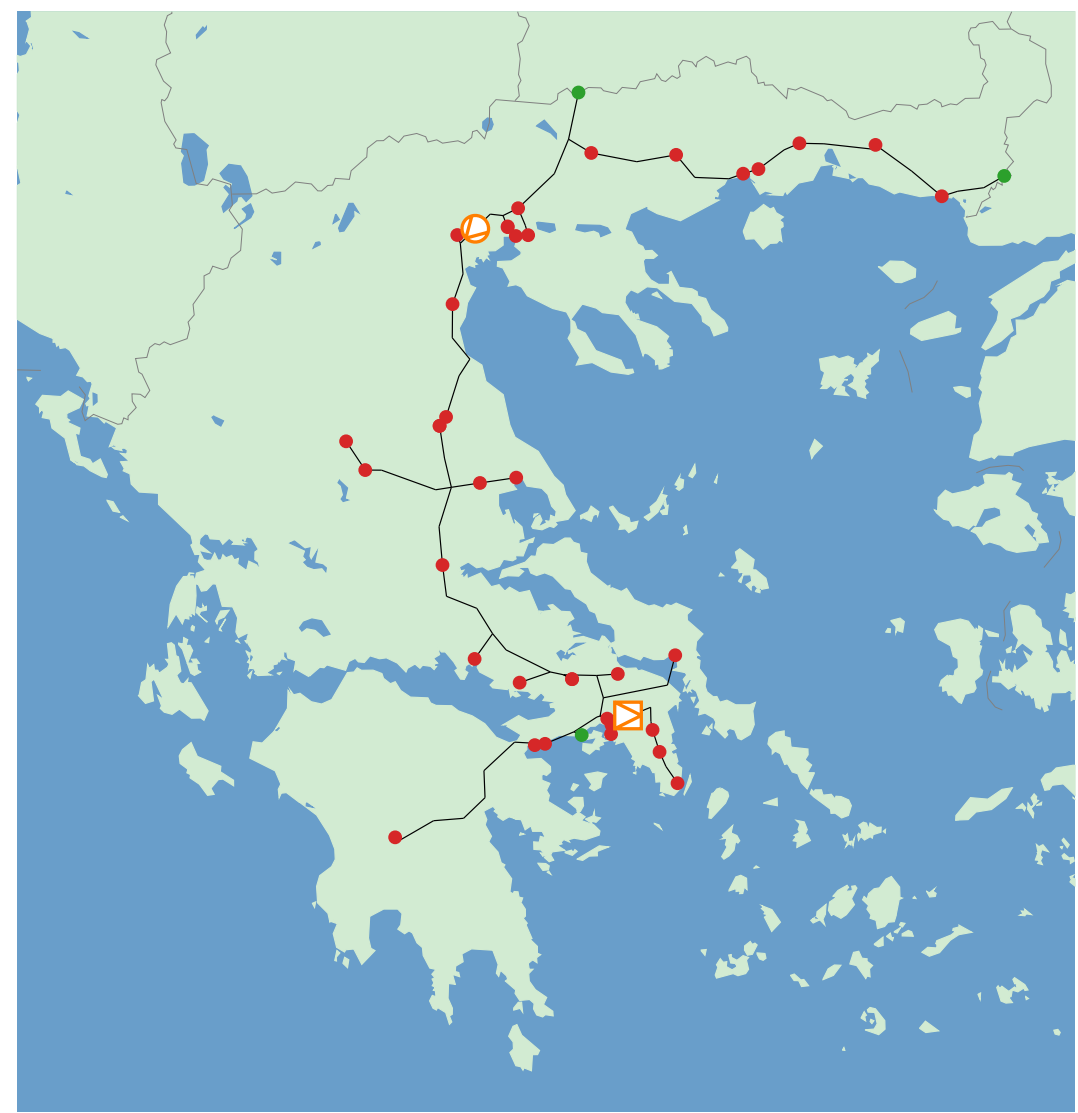

Figure 4.16: Greek natural gas transport network with continent (light green) and sea (blue). 3 entry nodes (green), 45 exit nodes (red), 1 control valve (orange symbol in the south), 1 compressor machine (orange symbol in the north), and 86 pipes (black). The 86 innodes are not visualized explicitly.

\section{Data}

We describe the main network properties of the Greek natural gas transport network; see Figure 4.16.

The network is made up of 134 nodes ( 3 entries, 45 exits, 86 inner nodes) and 133 arcs (86 pipes, 45 short pipes, 1 control valve, 1 compressor). We remark that short pipes are auxiliary elements that are essentially pipes $a=(u, w) \in A_{\mathrm{pi}}$ with $p_{u}=p_{w}$. Considering Figure 4.16, we see the active elements, i.e., the compressor machine in the north and the control valve in the south. We remark that the exit nodes are located closely together partially. Consequently, we are not able to distinguish all 
of them on the map. In general, the exit nodes are distributed over the whole country. The three entry nodes are distinguishable on the map. Two of them are located in the north, whereas the third one is located in the south. Note that the considered network is a tree.

Daily nomination data ranging from $11 / 01 / 2011$ to $02 / 17 / 2016$ is available on the DESFA website; see DESFA [47]. 336 days in this range have imbalanced nominations or other invalid data yielding an instance set of 1234 remaining days.

We processed the data and made it available online as GasLib-134 instance. ${ }^{1}$ The net-file describes the network and its parameters, while the scn-files describe the 1234 nominations. Detailed information about the Greek compressor machine are not publicly available. Hence, we use the data of the compressor machine compressor_1 of the publicly available instance GasLib-135. We provide more detailed explanations about the data and about the schemas of the xml-files in our following article:

[140] M. Schmidt, D. Aßmann, R. Burlacu, J. Humpola, I. Joormann, N. Kanelakis, T. Koch, D. Oucherif, M. E. Pfetsch, L. Schewe, R. Schwarz, and M. Sirvent. "GasLib-A Library of Gas Network Instances." In: Data 2.4 (2017). DOI: 10.3390/data2040040.

All flow values are given as volumetric flow, i.e., in $\mathrm{m}^{3} \mathrm{~h}^{-1}$. We use $0.7433 \mathrm{~kg} \mathrm{~m}^{-3}$ as norm density of natural gas to calculate the corresponding mass flow. Note that we use a more exact formula inside of the compressor model; see (3.15). Moreover, the specific gas constant is not given in the data. We set $\hat{R}_{\mathrm{S}}=500.27 \mathrm{~m}^{2} \mathrm{~s}^{-2} \mathrm{~K}^{-1}$ and use it for the pipe model and for the compressor model.

For the subsequent calculation, we remark that we increase all supplied and discharged flows by a factor of 2 in order to increase the nonlinearity and thus the overall hardness of the problem. As the maximum flow bounds of the three entries are tight, we manually set the maximum entry bounds to a very high value. Otherwise, the increase of all supplied and discharged flows by a factor of 2 would immediately yield lots of infeasibilities.

\section{Results for Algorithm 4.1}

We test Algorithm 4.1 with tolerances $\varepsilon=1 \times 10^{-3}$ and $\varepsilon=1 \times 10^{-4}$. The values are scaled to denote error in bar for the pressure loss on pipes and refer to $\ell_{2}$ - $\varepsilon$-feasibility; see Definition 4.3.2.

${ }^{1}$ http://gaslib.zib.de 
Table 4.1: Overview of the results of Algorithm 4.1 grouped by infeasible instances ("inf."), optimal instances with deactivated compressor ("opt."), and optimal instances with activated compressor ("opt. (compr.)"). Number of instances ("\#") and averages of iterations (" $\varnothing k ")$, total runtimes (" $\varnothing$ Total"), master problem runtimes (" $\varnothing$ Master") and subproblem runtimes (" $\varnothing$ Sub"). All runtimes are given in seconds.

\begin{tabular}{clrrrrr}
\hline$\varepsilon$ & Status & $\#$ & $\varnothing k$ & $\varnothing$ Total & $\varnothing$ Master & $\varnothing$ Sub \\
\hline \multirow{2}{*}{$1 \times 10^{-3}$} & inf. & 501 & 1.094 & 0.693 & 0.002 & 0.690 \\
& opt. & 179 & 2.508 & 2.932 & 0.021 & 2.911 \\
& opt. (compr.) & 554 & 2.570 & 6.987 & 0.015 & 6.972 \\
\hline \multirow{2}{*}{$1 \times 10^{-4}$} & inf. & 514 & 1.117 & 1.901 & 0.003 & 1.898 \\
& opt. & 175 & 2.897 & 4.009 & 0.025 & 3.984 \\
& opt. (compr.) & 545 & 2.901 & 13.370 & 0.017 & 13.353 \\
\hline
\end{tabular}

We use Gurobi 6.5.0 with default settings for the master problem $(\mathrm{M}(k))$ and Baron 12.3.3 to solve the small subproblems $(\mathrm{S}(i, k))$. Baron guarantees global solutions that are necessary for the theorems in Section 4.4. We set the solution of the master problem with objective value 0 as initial value for the subsequent NLPs in the subproblem. Furthermore, we set the Baron tolerance parameters optca and optcr to $1 \times 10^{-6}$ in the case of $\varepsilon=1 \times 10^{-3}$ and to $1 \times 10^{-8}$ for in the case of $\varepsilon=1 \times 10^{-4}$. We need these exact solutions for the subproblems as inaccurate solutions due to rounding errors may corrupt the property of cutting of the solution of the master problem; see Remark 4.8.2 and Theorem 4.4.6. This hampers finite termination.

Table 4.1 gives a basic overview of the results in terms of average numbers. Our algorithm solves all instances. For $\varepsilon=1 \times 10^{-3}$, approximately $59.4 \%$ of the instances are solved to global optimality, whereas $\sim 40.6 \%$ are proven to be infeasible by our method. In the case of $\varepsilon=1 \times 10^{-4}$, the numbers are slightly different, i.e., $\sim 58.3 \%$ and $\sim 41.7 \%$ for global optimality and infeasibility, respectively. The number of infeasible instances is less for $\varepsilon=1 \times 10^{-3}$ compared to $\varepsilon=1 \times 10^{-4}$; see third column. The fourth column shows that infeasibility is detected faster than global optimality is proven and that the iteration numbers are higher for $\varepsilon=1 \times 10^{-4}$ compared to $\varepsilon=1 \times 10^{-3}$. Additionally, the fifth column states the average runtime. The runtimes are higher for instances that are solved to global optimality and higher in the case of $\varepsilon=1 \times 10^{-4}$. The last two columns state the allocation of the runtime to master problem and subproblem and clarify that almost all of the time is spent for solving the subproblems. 


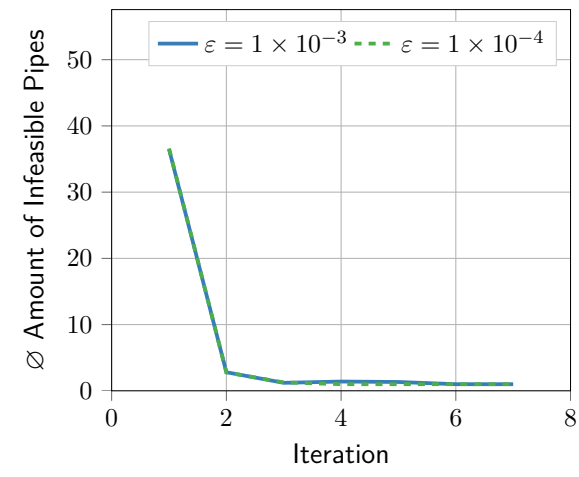

(a) Amount of infeasible pipes.

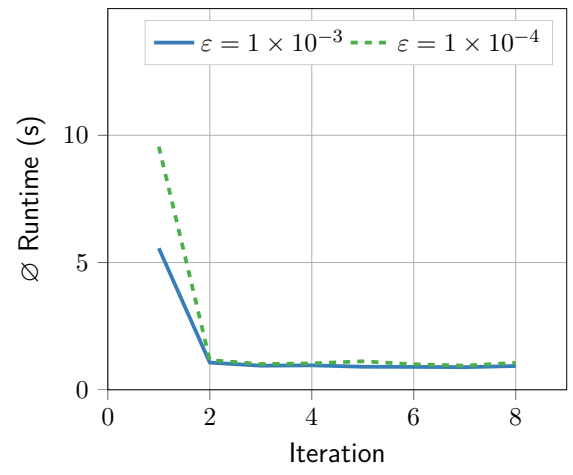

(b) Runtime.

Figure 4.17: Subproblem averages over iterations for Algorithm 4.1.

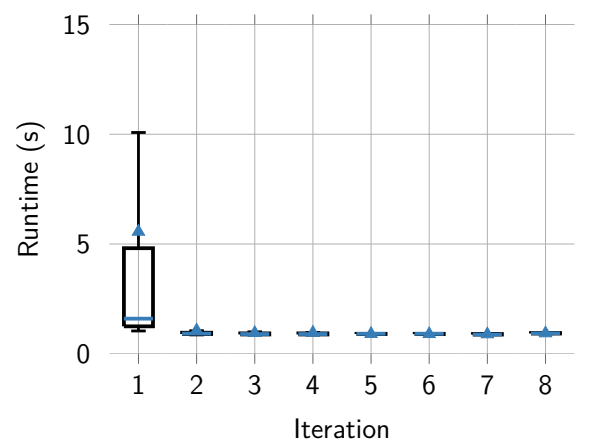

(a) Runtime.

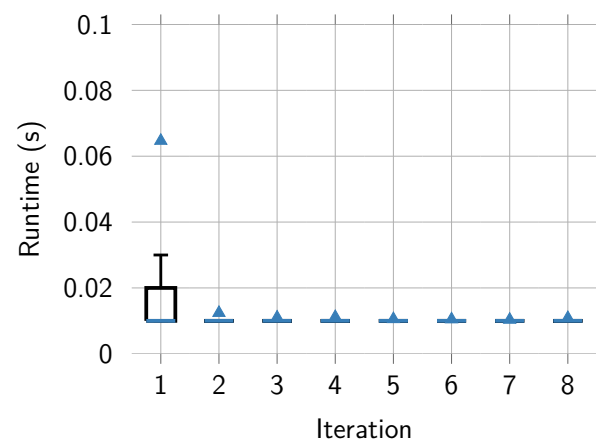

(b) Single runtimes.

Figure 4.18: Subproblem boxplots over iterations for Algorithm 4.1 with $\varepsilon=1 \times 10^{-3}$. The triangles represent the mean value.

Figure 4.17a shows the decreasing average amount of detected infeasible pipes over iterations towards optimality or infeasibility. As the runtime of the subproblem consumes the majority of the overall runtime, we investigate how the runtime distributes over the iterations in detail. Figure 4.17b shows the decreasing average runtime over iterations and Figure 4.18a shows the corresponding boxplots for $\varepsilon=1 \times 10^{-3}$. The boxplots for $\varepsilon=1 \times 10^{-4}$ look the same in terms of quality. We can see that almost all of the time is consumed in the first iteration. From iteration 2 on, Baron solves the 86, which is the number of pipes, NLPs almost always in preprocessing. The main runtime proportion is accumulated in iteration 1 . We remark that the averages for Figure 4.17b and Figure 4.18a are calculated by summing up 


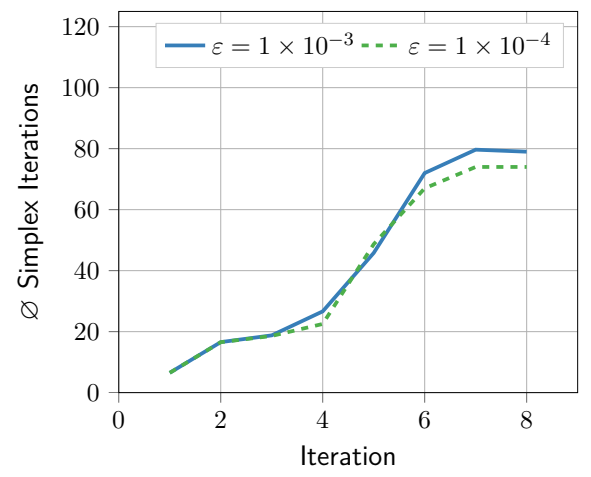

(a) Simplex iterations.

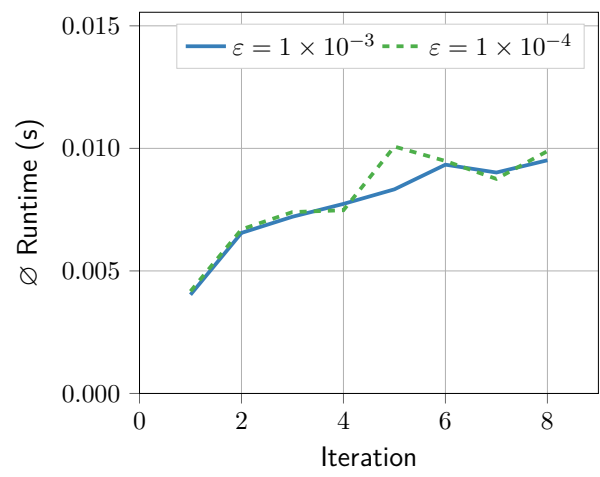

(b) Runtime.

Figure 4.19: Master problem averages over iterations for Algorithm 4.1.

the 86 NLP runtimes in every iteration for every instance. If we analyze the single NLPs and take their average for every iteration, we obtain the boxplot in Figure 4.18b. For the first iteration, we can see that $75 \%$ of the NLPs are solved in less than $\sim 0.02$ seconds. Nevertheless, single outliers raise the mean value to more than 0.06 seconds.

For the master problems, the runtimes are small compared to the subproblems; see Table 4.1. We plot average simplex iterations and average runtimes of the master problems, i.e. the MIPs, in every iteration in Figure 4.19a and Figure 4.19b, respectively. We see that both grow over the course of iterations.

\section{Results for Algorithm 4.2 and Algorithm 4.3}

We test Algorithm 4.2 and Algorithm 4.3 with tolerance $\varepsilon=1 \times 10^{-2}$. The value is scaled to denote error in bar for the pressure loss on pipes and refers to $\varepsilon$-feasibility; see Definition 4.3.3.

Remark 4.9.4. In our application, we obtain an exact oracle for function $f$ instead of the inexact function $g$ for Algorithm 4.3. To simulate this setting, we use a value $\varepsilon^{f}$ and sample values $\varepsilon_{i}^{f}(\cdot) \in\left[-\varepsilon^{f}, \varepsilon^{f}\right]$ for all $i \in[\sigma]$.

We select $\varepsilon^{f}=1 \times 10^{-3}$ and $\varepsilon^{f}=2 \times 10^{-3}$ for Algorithm 4.3. Note that Algorithm 4.2 can be interpreted as Algorithm 4.3 with $\varepsilon^{f}=0$.

We use Gurobi 6.5.0 with default settings for the master problem $(\mathrm{M}(k))$ and Ipopt 24.1.3 to solve the small subproblems $\left(\mathrm{S}^{\prime}(i, k)\right)$ and $\left(\mathrm{S}^{\prime \prime}(i, k)\right)$, which are the same in our framework; see Remark 4.9.4. We set the 
Table 4.2: Overview of the results of Algorithm $4.2\left(\varepsilon^{f}=0\right)$ and Algorithm 4.3 $\left(\varepsilon^{f}=1 \times 10^{-3}\right.$ and $\left.\varepsilon^{f}=2 \times 10^{-3}\right)$ grouped by infeasible instances ("inf."), optimal instances with deactivated compressor ("opt."), and optimal instances with activated compressor ("opt. (compr.)"). Number of instances ("\#") and averages of iterations (" $\varnothing k ")$, total runtimes (" $\varnothing$ Total"), master problem runtimes (" $\varnothing$ Master") and subproblem runtimes (" $\varnothing$ Sub"). All runtimes are given in seconds.

\begin{tabular}{clrrrrr}
\hline$\varepsilon^{f}$ & Status & $\#$ & $\varnothing k$ & $\varnothing$ Total & $\varnothing$ Master & $\varnothing$ Sub \\
\hline \multirow{2}{*}{0} & inf. & 603 & 1.602 & 0.376 & 0.012 & 0.364 \\
& opt. & 151 & 9.298 & 2.136 & 0.397 & 1.740 \\
& opt. (compr.) & 480 & 17.746 & 4.126 & 1.103 & 3.022 \\
\hline \multirow{2}{*}{$1 \times 10^{-3}$} & inf. & 597 & 1.610 & 0.366 & 0.014 & 0.352 \\
& opt. & 155 & 10.658 & 2.543 & 0.559 & 1.984 \\
& opt. (compr.) & 482 & 19.600 & 4.789 & 1.429 & 3.360 \\
\hline \multirow{2}{*}{$2 \times 10^{-3}$} & inf. & 593 & 1.612 & 0.377 & 0.014 & 0.362 \\
& opt. & 159 & 13.258 & 3.217 & 0.908 & 2.309 \\
& opt. (compr.) & 482 & 23.064 & 7.413 & 3.346 & 4.067 \\
\hline
\end{tabular}

solution of the master problem with objective value 0 as initial value for the subsequent NLPs in the subproblem. Ipopt is the state-of-the-art solver to solve NLPs, but we remark that it does not necessarily yield global optimal solutions. Nevertheless, Algorithm 4.2 and Algorithm 4.3 do not rely on global solutions for the subproblems. The arguments for a finite number of iterations consisting of volume reduction and interval reduction hold for feasible points as well; see Remark 4.8.4

Table 4.2 gives a basic overview of the results in terms of average numbers. Our algorithms solve all instances. Depending on the parameter $\varepsilon^{f}$, approximately $48 \%$ to $49 \%$ of the instances are proven to be infeasible. On the other hand, $\sim 51 \%$ to $52 \%$ of the instances are solved to global optimality. The number of infeasible instances increases for smaller $\varepsilon^{f}$; see third column. Again, the fourth column shows that infeasibility is detected with significantly less iterations than global optimality is proven. Moreover, the fifth column shows that the runtimes are higher for instances that are solved to global optimality and higher in the case of higher $\varepsilon^{f}$. The distribution of the runtimes amongst master problems and subproblems in the last two columns shows that more time is spent to solve the subproblems. Nevertheless, the discrepancy is smaller than in Table 4.1.

Figure 4.20a shows the decreasing average amount of detected infeasible pipes over iterations towards optimality or infeasibility. We remark 


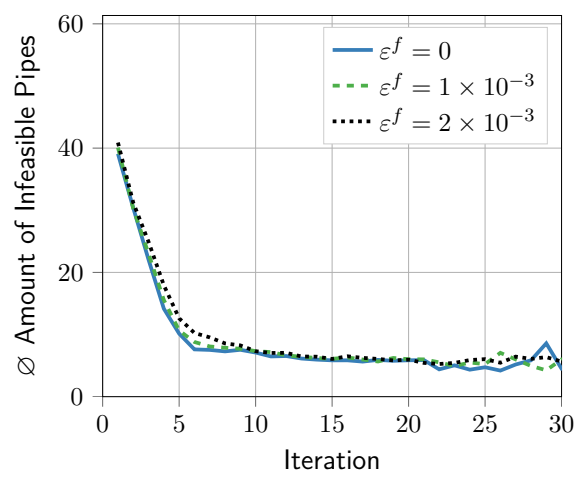

(a) Amount of infeasible pipes.

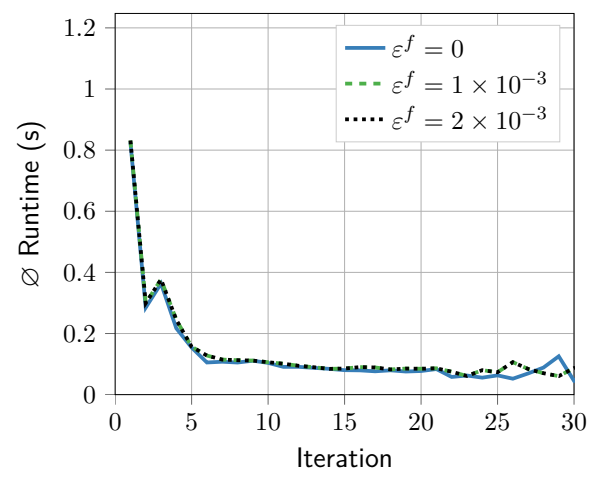

(b) Runtime.

Figure 4.20: Subproblem averages over iterations for Algorithm 4.2 and Algorithm 4.3. The $x$-axis is truncated at iteration 30 .

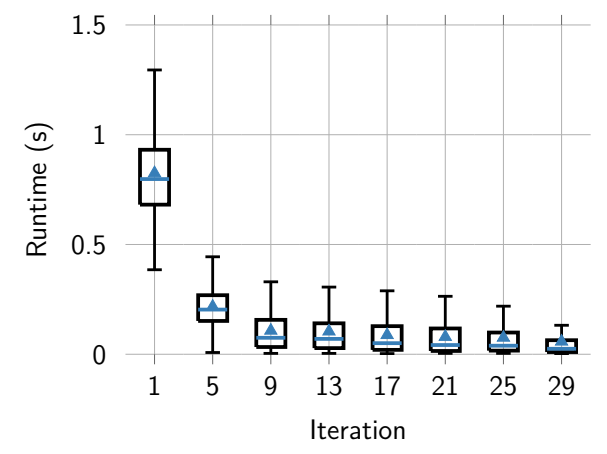

(a) Runtime.

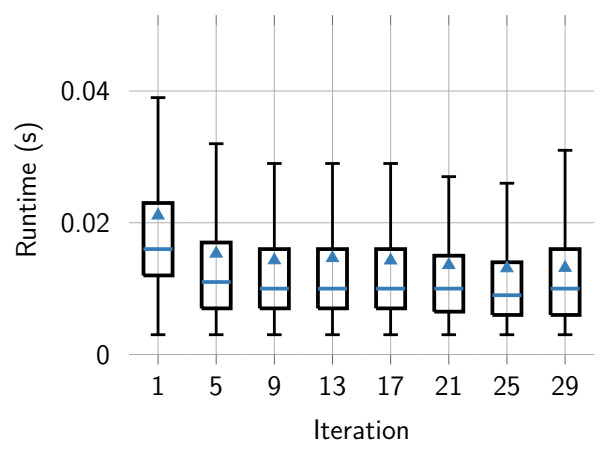

(b) Single runtimes.

Figure 4.21: Subproblem boxplots over selected iterations for Algorithm 4.2. The triangles represent the mean value.

that we truncate all $x$-axes at iteration 30 . Again, as the runtime of the subproblem consumes a large share of the overall runtime, we investigate how the runtime distributes over the iterations in detail. Figure $4.20 \mathrm{~b}$ shows the decreasing average runtime over iterations and Figure 4.21a shows corresponding selected boxplots for Algorithm 4.2, i.e., for $\varepsilon^{f}=0$. The boxplots for $\varepsilon^{f}=1 \times 10^{-4}$ and $\varepsilon^{f}=2 \times 10^{-4}$ look the same in terms of quality. Again, we can see that a lot of time is consumed in the first iteration. Note that the averages for Figure $4.20 \mathrm{~b}$ and Figure $4.21 \mathrm{a}$ are calculated by summing up the NLP runtimes for the infeasible pipes in every iteration for 


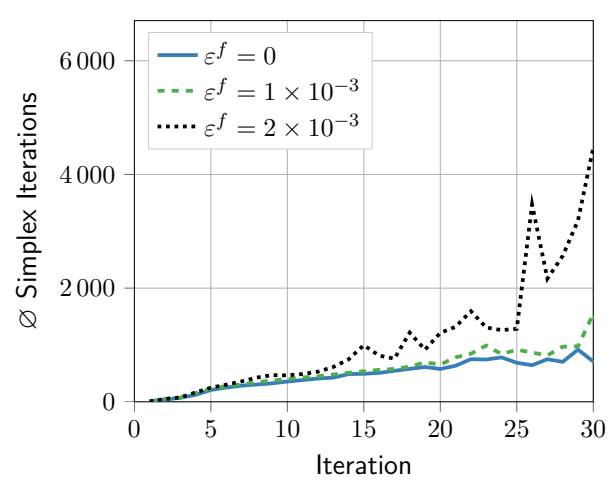

(a) Simplex iterations.

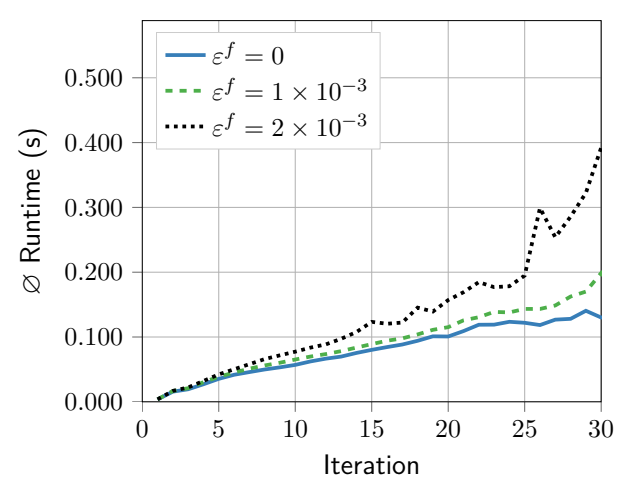

(b) Runtime.

Figure 4.22: Master problem averages over iterations for Algorithm 4.2 and Algorithm 4.3. The $x$-axis is truncated at iteration 30 .

every instance again. If we analyze the single NLPs and take their average for every iteration, we obtain the boxplot in Figure 4.21b.

For the master problems, the runtimes are small compared to the subproblems; see Table 4.2 -we remark that the difference is not that huge as in Table 4.1. We plot average simplex iterations and average runtimes of the master problems, i.e., the MIPs, in every iteration in Figure 4.22a and Figure $4.22 \mathrm{~b}$, respectively. Again, we see that both grow over the course of iterations.

\section{Remarks for Algorithms 4.1-4.3}

In this paragraph, we state remarks to classify the results. Moreover, we permit ourselves to interpret some findings.

Remark 4.9.5 (Complexity of Master Problems). The MIP s grow from iteration to iteration due to added cut inequalities and additional binary variables. As we never add more than one discretization point per iteration and pipe, this growth is bounded from above. The size of the MIP, in terms of constraints and variables, grows linearly over the course of the iterations; see Remark 4.8.3 as well. This linear growth translates into an exponential increase in simplex iterations and runtime in theory. This fact cannot be avoided when solving MIPs as it is due to the exponential worst case complexity of MIPs themselves; see Section 2.1 and Section 2.3.

In practice, the exponential growth is not apparent for Algorithm 4.1, which may be due to small iteration numbers, small runtimes, or a small 
amount of instances; see Figure 4.19. On the other hand, the exponential growth can be seen for the example of $\varepsilon^{f}=2 \times 10^{-3}$ for Algorithm 4.3; see Figure 4.22. This may be due to larger iteration numbers. Remember that we truncate the $x$-axis at iteration 30 . The values get huge afterward and emphasize the exponential growth. We truncate the figure because these huge values hamper the overview.

Remark 4.9.6 (Complexity of Subproblems). For Algorithm 4.1 the number of independent $\mathrm{NLP} s$ to be solved is constant over all iterations and equals the number of pipes (86) in the network. For Algorithm 4.2 and Algorithm 4.3 the number of independent NLPs to be solved depends on the number of infeasible pipes. Thus, the amount of $\mathrm{NLP} s$ in every iteration is bounded from above by the number of pipes (86) in the network and does not grow over the course of iterations.

Remark 4.9.7 (Comparison of Baron and Ipopt for the Subproblems). For Algorithm 4.1 and with Baron, we solve the same amount of subproblems in every iteration; see Remark 4.9.6. Nevertheless, the NLPs of the first iteration are harder to solve and yield higher runtimes; see Figure 4.18. These outliers may lead to exploding runtimes for single instances.

On the other hand, the NLPs of the subproblem for Algorithm 4.2 and Algorithm 4.3 solved by lpopt depend on the number of infeasible pipes; see Remark 4.9.6 again. The decreasing amount of infeasible pipes goes in parallel with the decreasing runtime; see Figure 4.20. The NLP solution runtimes of the subproblem solved by lpopt are smooth. All runtimes lie in a similar area and all $\mathrm{NLP} s$ are solved quickly; see Figure 4.21b. This behavior is preferable as it guarantees robustness for arbitrary instances.

The observations make sense as Baron solves the NLPs to global optimality, whereas Ipopt is a local NLP solver. This is in line with our algorithmic framework; see Remark 4.8.4.

Remark 4.9.8 (Small Iteration Numbers for Algorithm 4.1). The required iteration numbers for Algorithm 4.1 are quite low for the considered class of gas transport problems; see Table 4.1. The reason is that the relation of in-and outflow pressures according to pipe model (4.34) on tree-like gas transport problems has rather mild nonlinearities.

This can be seen in Figure 4.23 where the refined relaxations of this nonlinearity are shown over the course of iterations. The initial relaxation after preprocessing is displayed in Figure 4.23a. On a large scale, this relaxation already seems to be quite tight. It turns out that the optimal solution lies around an inflow pressure of approximately 48 bar. A zoom 


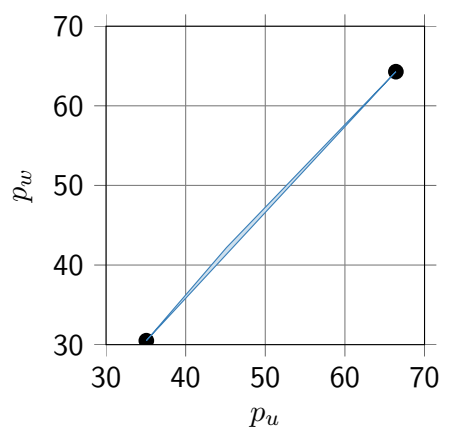

(a) After preprocessing.

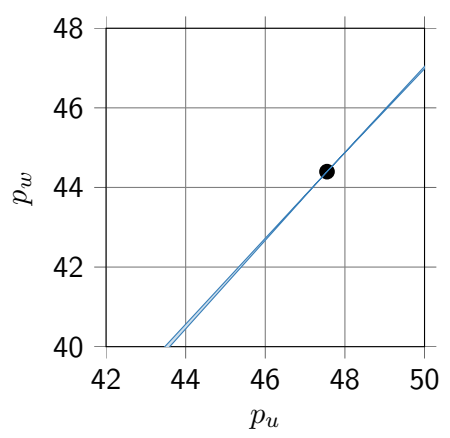

(c) Zoomed area after 1st iteration.

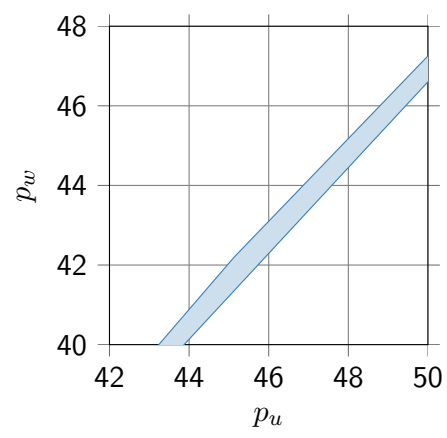

(b) Zoomed area after preprocessing.

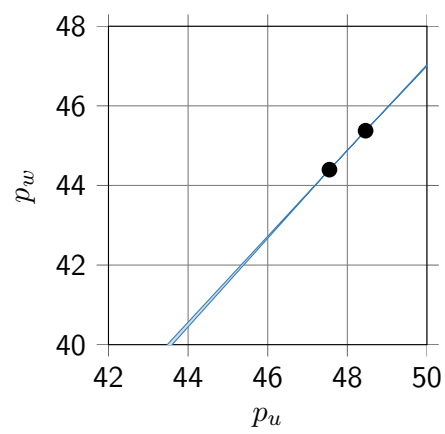

(d) Zoomed area after 2nd iteration.

Figure 4.23: Tightened relaxation of in- and outflow pressure over the course of iterations.

into this region is shown in Figure 4.23b. It can be seen that the possible error is $\sim 0.5$ bar to 0.75 bar, which is still large compared to the tolerance of $1 \times 10^{-3}$ bar or $1 \times 10^{-4}$ bar. However, the next iterations directly give tight relaxations that yield the reported low iteration numbers; see Figure 4.23c and Figure 4.23d.

Note that the iteration numbers for Algorithm 4.2 and Algorithm 4.3 are higher as the constructed quadrilaterals and hexagons do not yield such tight relaxations.

Remark 4.9.9 (Comparison of Different $\varepsilon^{f}$ for Algorithm 4.2 and Algorithm 4.3). Table 4.2 shows that the larger $\varepsilon^{f}$, the less infeasible instances we detect. This is to be expected as the feasible set of the master problem grows with larger $\varepsilon^{f}$; see Figure 4.11.

Moreover, we remark that higher values for $\varepsilon^{f}$ yield more infeasible pipes, i.e., more added linearization points for the MIP in the master problem; 
see Figure 4.20a-especially from iteration 2 to 10. As already discussed in Remark 4.9.5, these linearization points yield larger master problem MIPs. Accordingly, the corresponding instances are harder to solve in general as it can be seen in Table 4.2 and Figure 4.22.

Although nonconvex MINLP problems from gas transport are known to be hard to solve, Algorithms 4.1-4.3 solve all instances on a real-world network on a desktop computer, which shows the applicability of our approaches. Referring to the master problem, it is clear that our algorithms are particularly strong for instances that can be solved within a small number of iterations in which the exponential growth of MIP complexity does not become apparent; see Remark 4.8.3 and Remark 4.9.5. On the other hand, the complexity of the subproblem is constant and does not suffer from a large number of iterations; see Remark 4.9.6. Nevertheless, a global NLP solver as Baron may complicate the solution process, whereas a local NLP solver as Ipopt is more stable; see Remark 4.9.7. 



\section{Instantaneous Control for PDE-Constrained Mixed-Integer Programs}

In this chapter, we present an instantaneous control algorithm to solve problems that combine discrete decisions and systems of PDEs where the solution is depending on time and space. We use this general approach for the transient case of gas transport problems and show promising numerical results. To this end, we discretize the space-time dependent Euler PDE system on a graph in a suitable way that allows us to derive solutions analytically. The time discretization predetermines a time decomposition with single problems for every time step. Our main contribution is a new mixed implicit-explicit Euler discretization that allows us to formulate MIP models in every time step of the proposed instantaneous control method. Taken all together, we rely on our guidelines of decomposing our problem and using MIP solvers as a workhorse again.

Chapter 5 is structured as follows. In Section 5.1, we introduce the problem under consideration and discuss an instantaneous control approach. Afterward, we provide a literature survey in Section 5.2 and make additional remarks in Section 5.3. In Section 5.4, we then adapt the approach for transient gas transport problems, present the discretizations, and provide promising numerical results.

We finally state that significant parts of this chapter are published in an international journal; see our article Gugat et al. [76]. The author of this thesis made significant contributions for conceiving, developing, and elaborating all the presented contents that follow. In addition, we explicitly state results that originate from co-authors and where the author of this thesis did not contribute. 


\subsection{Problem Statement and Derivation of the Algorithm}

The problem we consider incorporates discrete decisions and is constrained by a system of PDEs where the solution is depending on time and space. We assume that we are dealing with a problem on a graph and consider the abstract problem

$$
\begin{array}{ll}
\min _{x} & c^{\top} x \\
\text { s.t. } & A x \geq b, \quad x^{-} \leq x \leq x^{+}, \quad x_{\mathcal{C}} \in \mathbb{R}^{|\mathcal{C}|}, \quad x_{\mathcal{I}} \in \mathbb{Z}^{|\mathcal{I}|}, \\
& x_{\mathcal{C}} \in \mathcal{X},
\end{array}
$$

derived from Problem (1.1). In more detail, $x_{\mathcal{C}} \in \mathcal{X}$ comprises PDE-constraints. Moreover, we assume that the objective function (5.1a) is linear, e.g., due to linearization of (1.1a). To the best of our knowledge, no general-purpose solution strategies exist to directly tackle such mixed-integer PDE-constrained problems; see the recent survey Hante et al. [83] for more details about this and related problem classes.

One remedy is to semi-discretize the PDEs, i.e., to discretize the time horizon $\left[0, \mathcal{T}^{\max }\right]$. To this end, we use discretization time points $\mathcal{T}:=\left\{0=t_{0}, t_{1}, \ldots, t_{K}=\mathcal{T}^{\max }\right\}$. The resulting problem is still an MIP that is "only" constrained by a system of ODEs now. Depending on the applied time discretization, this ODE constrained problem is still out of reach for general-purpose methods. Even for this discrete-time optimization problem, no published algorithm seems to be available.

The situation changes if we consider optimization problems that arise by decomposing the discretized time steps and considering the time steps separately. Thus, we define the optimization problems

$$
\begin{array}{ll}
\min _{x^{t}} & c^{\top} x^{t} \\
\text { s.t. } & A x^{t} \geq b, \quad x^{-} \leq x^{t} \leq x^{+}, \quad x_{\mathcal{C}}^{t} \in \mathbb{R}^{|\mathcal{C}|}, \quad x_{\mathcal{I}}^{t} \in \mathbb{Z}^{|\mathcal{I}|}, \\
& x_{\mathcal{C}}^{t} \in \mathcal{X}^{t},
\end{array}
$$

for all $t \in \mathcal{T}$ where $x^{t}$ refers to the variables of time step $t$. We now consider a general instantaneous control algorithm that is stated in an abstract manner in Algorithm 5.1. We decompose Problem (5.1) along the time discretizations, obtain Problem (5.2) for every time point $t_{\kappa+1}$ with $\kappa \in[K-1]:=\{0, \ldots, K-1\}$, and solve the problems step by step. To do so, we need initial conditions that are feasible for Problem (5.2). Note that 
Algorithm 5.1 Instantaneous Control Algorithm

Input: A PDE-constrained mixed-integer problem (5.1), a discretized time horizon, a semi-discretization scheme for the PDEs, and feasible initial conditions $\widetilde{x}^{t_{0}}$.

Output: Feasible solution $x$ or an indication that no feasible solution can be found.

1: for $\kappa \in[K-1]$ do

2: $\quad$ Setup the optimization problem (5.2) for time step $t_{\kappa+1}$ that only depends on the state at the previous time step $t_{\kappa}$ and solve (5.2).

3: $\quad$ if Problem (5.2) is infeasible then

4: return "No feasible solution can be found".

5: $\quad$ else

6: $\quad$ Store optimal solution $\widetilde{x}^{t_{\kappa+1}}$.

7: end if

8: end for

9: return Feasible solution $\widetilde{x}=\left(\left(\widetilde{x}^{t_{0}}\right)^{\top},\left(\widetilde{x}^{t_{1}}\right)^{\top}, \ldots,\left(\widetilde{x}^{t_{K-1}}\right)^{\top},\left(\widetilde{x}^{t_{K}}\right)^{\top}\right)^{\top}$.

Line 2 mentions that Problem (5.2) only depends on the time step before for a certain time step. This dependency is modeled inside of $\mathcal{X}^{t}$ in (5.2c).

There are various open questions regarding Algorithm 5.1. In order to concretize Problem (5.2), we have to specify the linear objective function (5.2a), the linear constraints $(5.2 \mathrm{~b})$ and the constraints $(5.2 \mathrm{c})$. We note that we still want to use MIP solvers as a workhorse. Consequently, we want to obtain (5.2c) as a linear model. In order to do so, the specific time discretization have to be discussed. Obviously, there is a large number of possible discretizations. As this strongly depends on the PDE itself, we derive an approach for the Euler equations that arise from transient gas transport optimization; see Section 5.4. Beforehand, we provide a literature survey for instantaneous control and make further remarks about the algorithm; see Section 5.2 and Section 5.3, respectively.

\subsection{Literature Survey: Instantaneous Control}

Instantaneous control is frequently used for challenging control problems; see, e.g., Choi et al. $[28,29]$ for instantaneous control of backward-facing step flows, Altmüller et al. [2] for the control of linear wave equations, Hinze [85] for the control of wave equations in networks, Hundhammer 
and Leugering [90] for instantaneous control of vibrating string networks, or Herty et al. [84] for an application to traffic flows. Recently, a similar approach for mathematical programs with equilibrium constraints (MPEC) combined with optimal control problems has been presented in Antil et al. [3].

To the best of our knowledge, there is no published research on instantaneous control of MIPs with hyperbolic PDEs on graphs. For that purpose, we study the case of transient gas transport optimization in Section 5.4.

\subsection{Further Remarks}

We make further remarks on instantaneous control.

Remark 5.3.1 (Feasibility vs. Infeasibility). In Algorithm 5.1, we solve Problem (5.2) for a certain time step only depending on the time step before. Thus, the problems are solved step by step. It is obvious that such an approach cannot guarantee global optimality of Problem (5.1) in general. Hence, if Algorithm 5.1 stops at Line 9, it returns a feasible solution. Additionally, if Algorithm 5.1 stops at Line 4, no statement about infeasibility can be made.

Remark 5.3.2 (Possible Extensions-Larger Time Windows). The instantaneous control method is a special case of a model predictive or receding horizon control and one drawback is the minimum time window size. A possible extension is to widen the view and to solve problems for several time steps simultaneously. On the one hand, this possibly improves solution qualities but on the other hand, we are faced with harder problems.

Remark 5.3.3 (Instantaneous Control Algorithms for Domain Decomposition). There is an additional article where we use instantaneous control together with domain decomposition:

[106] G. Leugering, A. Martin, M. Schmidt, and M. Sirvent. "Nonoverlapping Domain Decomposition for Optimal Control Problems governed by Semilinear Models for Gas Flow in Networks." In: Control \&6 Cybernetics 46.3 (2017), pp. 191-225.

The content of the article is not part of this thesis.

\subsection{Application to Transient Gas Transport Optimization}

Algorithm 5.1 is developed for Problem (5.1). We apply this algorithm for transient gas transport optimization. In Section 5.4.1, an objective function 
is defined, while we derive a suitable transient pipe model in Section 5.4.2. In the latter, we explain the specific discretization techniques that allow us to formulate Problem (5.2) as an MIP. Finally, we present promising numerical results in Section 5.4.3.

\subsubsection{Objective Function}

The objective function (5.2a) is linear. There are many reasonable objective functions for transient gas transport optimization and we are interested in the question whether, given an initial state, the network can be controlled such that another prescribed state can be reached in a certain amount of time. This question is motivated by the desire to satisfy a given demand and related to problems of exact nodal controllability, see, e.g., Gugat et al. [73]. As the aim is to track the desired state, this leads us to tracking-type objective functions. To this end, let

$$
\hat{p}_{u}, \hat{q}_{u} \quad \text { for all } u \in V_{+} \cup V_{-}
$$

describe the pressure and mass flow to be reached at entry and exit nodes. Moreover, we define

$$
\eta_{u}, \mu_{u} \quad \text { for all } u \in V_{+} \cup V_{-}
$$

as node specific penalty parameters for pressure and mass flow. Taken all together, the tracking-type objective function to be minimized reads

$$
\left\|\left(\eta_{u}\left(p_{u}^{t}-\hat{p}_{u}\right)\right)_{u \in V_{+} \cup V_{-}}\right\|+\left\|\left(\mu_{u}\left(q_{u}^{t}-\hat{q}_{u}\right)\right)_{u \in V_{+} \cup V_{-}}\right\|
$$

with the terms in the norms being vectors of dimension $\left|V_{+}+V_{-}\right|$. The objective function (5.2a) is supposed to be linear. Hence, compatible vector norms to minimize (5.3) are the 1 - and $\infty$-norm as LP-models of these norms exist; see McCarl and Spreen [117, Chapter 9]. We explain these models.

To clarify an LP-model for the 1-norm, we assume a vector $x \in \mathbb{R}^{n}$. Generally speaking, a linear model for

$$
\begin{array}{cl}
\min & \|x\|_{1} \\
\text { s.t. } & x \in \mathbb{R}^{n}
\end{array}
$$

is wanted. We assume that (5.4) involves additional linear constraints that do not affect the LP-model property under discussion. By definition, 
$\|x\|_{1}$ equals $\sum_{i \in[n-1]}\left|x_{i}\right|$. The sum itself is obviously linear. Thus, a linear model for the absolute value is needed. For that reason, we add the linear constraints $x_{i}=x_{i}^{\text {pos }}-x_{i}^{\text {neg }}$ with $x_{i}^{\text {pos }}, x_{i}^{\text {neg }} \in \mathbb{R}_{+}$for all $i \in[n-1]$. Eventually, we replace $\left|x_{i}\right|$ by $x_{i}^{\text {pos }}+x_{i}^{\text {neg }}$ for all $i \in[n-1]$ to obtain an LP.

$$
\begin{array}{ll}
\min & \sum_{i=0}^{n-1}\left(x_{i}^{\mathrm{pos}}+x_{i}^{\mathrm{neg}}\right) \\
\text { s.t. } & x_{i}=x_{i}^{\mathrm{pos}}-x_{i}^{\mathrm{neg}} \quad \text { for all } i \in[n-1], \\
& x, x^{\mathrm{pos}}, x^{\mathrm{neg}} \in \mathbb{R}^{n} .
\end{array}
$$

We show that the reformulation is correct for all optimal solutions.

Lemma 5.4.1. If $\left(x^{*},\left(x^{\text {pos }}\right)^{*},\left(x^{\text {neg }}\right)^{*}\right)$ is an optimal solution for Problem (5.5), then $x^{*}$ is an optimal solution for Problem (5.4).

Proof. Without loss of generality, we fix the setting to the case $n=1$. Note that the reformulation (5.5) is only correct if $x_{i}^{\text {pos }}=0$ or $x_{i}^{\text {neg }}=0$. As a consequence, we have to show that all optimal solutions of Problem (5.5) have this property. Considering an arbitrary feasible solution of Problem (5.5), we show that there is always a solution with $x_{i}^{\text {pos }}=0$ or $x_{i}^{\text {neg }}=0$ and with better objective value. For this purpose, assume a feasible point of Problem (5.5) with $x_{0}^{\text {pos }}=a>0, x_{0}^{\text {neg }}=b>0$, and objective value $a+b$. For the first case, we assume $a \geq b$. In this case, a solution $x_{0}^{\mathrm{pos}}=a-b \geq 0$ and $x_{0}^{\text {neg }}=0$ with better objective value $a-b<a+b$ and with the required property can be constructed. For the second case, we assume $a<b$. In this case, a solution $x_{0}^{\text {pos }}=0$ and $x_{0}^{\text {neg }}=b-a>0$ with better objective value $b-a<a+b$ and with the required property can be constructed. Thus, all optimal solutions of Problem (5.5) have the required property as well.

To clarify an LP-model for the $\infty$-norm, we assume the vector $x \in \mathbb{R}^{n}$ again. Generally spoken, we want a linear model for

$$
\begin{array}{cl}
\min & \|x\|_{\infty} \\
\text { s.t. } & x \in \mathbb{R}^{n} .
\end{array}
$$

Again, we assume that (5.6) involves additional linear constraints that do not affect the LP-model property under discussion. By definition, $\|x\|_{\infty}$ equals $\max _{i \in[n-1]}\left|x_{i}\right|$. For the absolute value, we use the same reformulation as before. Eventually, we replace the maximum term by a new variable 
$z \in \mathbb{R}$ and add the constraints $x_{i}^{\text {pos }}+x_{i}^{\text {neg }} \leq z$ for all $i \in[n-1]$ to obtain a linear model.

$$
\begin{array}{ll}
\min & z \\
\text { s.t. } & x_{i}=x_{i}^{\text {pos }}-x_{i}^{\text {neg }} \quad \text { for all } i \in[n-1], \\
& x_{i}^{\text {pos }}+x_{i}^{\text {neg }} \leq z \quad \text { for all } i \in[n-1] \\
& x, x^{\text {pos }}, x^{\text {neg }} \in \mathbb{R}^{n}, z \in \mathbb{R} .
\end{array}
$$

We show that the reformulation is correct for all optimal solutions.

Corollary 5.4.2. If $\left(x^{*},\left(x^{\mathrm{pos}}\right)^{*},\left(x^{\mathrm{neg}}\right)^{*}, z^{*}\right)$ is an optimal solution for Problem (5.7), then $x^{*}$ is an optimal solution for Problem (5.6).

Proof. The reformulation for the absolute value is the same as in (5.5). Accordingly, Lemma 5.4.1 can be used again. Moreover, the representation of the maximum with a variable $z$ is obviously valid.

Taken all together, we can use the linear formulations (5.5) or (5.7) to model the minimization of the tracking-type objective function (5.3) with the 1 - and the $\infty$-norm.

\subsubsection{Transient Pipe Model}

We show how the constraints of Problem (5.2) correspond to the gas model (3.27). Remember that Problem (5.2) is defined for one time point. The mixed-integer linear part $(5.2 \mathrm{~b})$ is directly represented by $(3.27 \mathrm{a})-(3.27 \mathrm{~g})$, which is given for all $t \in \mathcal{T}$ separately. The nonlinear part $(5.2 \mathrm{c})$ is more complicated. It is determined by pipe model $(3.27 \mathrm{~h})$, which is our starting point.

We explain the derivation of the transient pipe model and show tailored time discretizations of the underlying Euler equations afterward.

\section{Derivation}

We start with pipe model $(3.27 \mathrm{~h})$ and simplify. Note that it consists of the isothermal Euler equations (3.25) and the definition of the compressibility factor $z$ in (3.23), which we assume to be constant. In this case, and analogous to (4.29), a constant speed of sound $\hat{c}$ with

$$
\hat{c}^{2}=\hat{R}_{\mathrm{s}} \hat{T} \hat{z}
$$


is obtained; see Figure 5.1. Thus, the pipe model reads

$$
\begin{aligned}
\partial_{t} p+\hat{c}^{2} \partial_{x} \chi & =0, \\
\partial_{t} \chi+\partial_{x}\left(p+\frac{\hat{c}^{2} \chi^{2}}{p}\right) & =-\frac{\hat{\theta}_{a} \hat{c}^{2}}{2} \frac{\chi|\chi|}{p}-\frac{\hat{g} \hat{s}_{a}}{\hat{c}^{2}} p .
\end{aligned}
$$

Additionally, (3.22) and (5.8) yield

$$
\rho=\frac{p}{\hat{c}^{2}} .
$$

Consequently, we can reformulated the term in $(5.9 \mathrm{~b})$ by

$$
p+\frac{\hat{c}^{2} \chi^{2}}{p} \stackrel{(3.24)}{=} p+\frac{\hat{c}^{2} \rho^{2} v^{2}}{p} \stackrel{(5.10)}{=} p+\frac{p v^{2}}{\hat{c}^{2}}=p\left(1+\frac{v^{2}}{\hat{c}^{2}}\right)
$$

and obtain

$$
\begin{aligned}
\partial_{t} p+\hat{c}^{2} \partial_{x} \chi & =0, \\
\partial_{t} \chi+\partial_{x}\left(p\left(1+\frac{v^{2}}{\hat{c}^{2}}\right)\right) & =-\frac{\hat{\theta}_{a} \hat{c}^{2}}{2} \frac{\chi|\chi|}{p}-\frac{\hat{g} \hat{s}_{a}}{\hat{c}^{2}} p .
\end{aligned}
$$

Finally, we assume very small velocities, i.e., $|v| \ll \hat{c}$, such that $1+\left(v^{2} / \hat{c}^{2}\right) \approx 1$ and arrive at our transient pipe model, a semi-linear model; see Osiadacz [124]:

$$
\begin{aligned}
\partial_{t} p+\hat{c}^{2} \partial_{x} \chi & =0, \\
\partial_{t} \chi+\partial_{x} p & =-\frac{\hat{\theta}_{a} \hat{c}^{2}}{2} \frac{\chi|\chi|}{p}-\frac{\hat{g} \hat{s}_{a}}{\hat{c}^{2}} p .
\end{aligned}
$$

Figure 5.1 illustrates the classification of this transient pipe model compared to our stationary pipe model used in Chapter 4. Note that similar figures to classify gas models are used in Joormann et al. [93].

\section{Time Discretizations}

We show how the involved variables $p_{u}^{t}, p_{w}^{t}, \chi_{a, u}^{t}$, and $\chi_{a, w}^{t}$ fit the transient pipe model (5.11) depending on the specific time-discretization that is used to semi-discretize the system. Moreover, we have to clarify how this fits the constraints $x_{\mathcal{C}}^{t} \in \mathcal{X}^{t}$ in $(5.2 \mathrm{c})$.

Obviously, there is a large number of possible discretizations and we concentrate on those that yield tractable models that are to be solved 


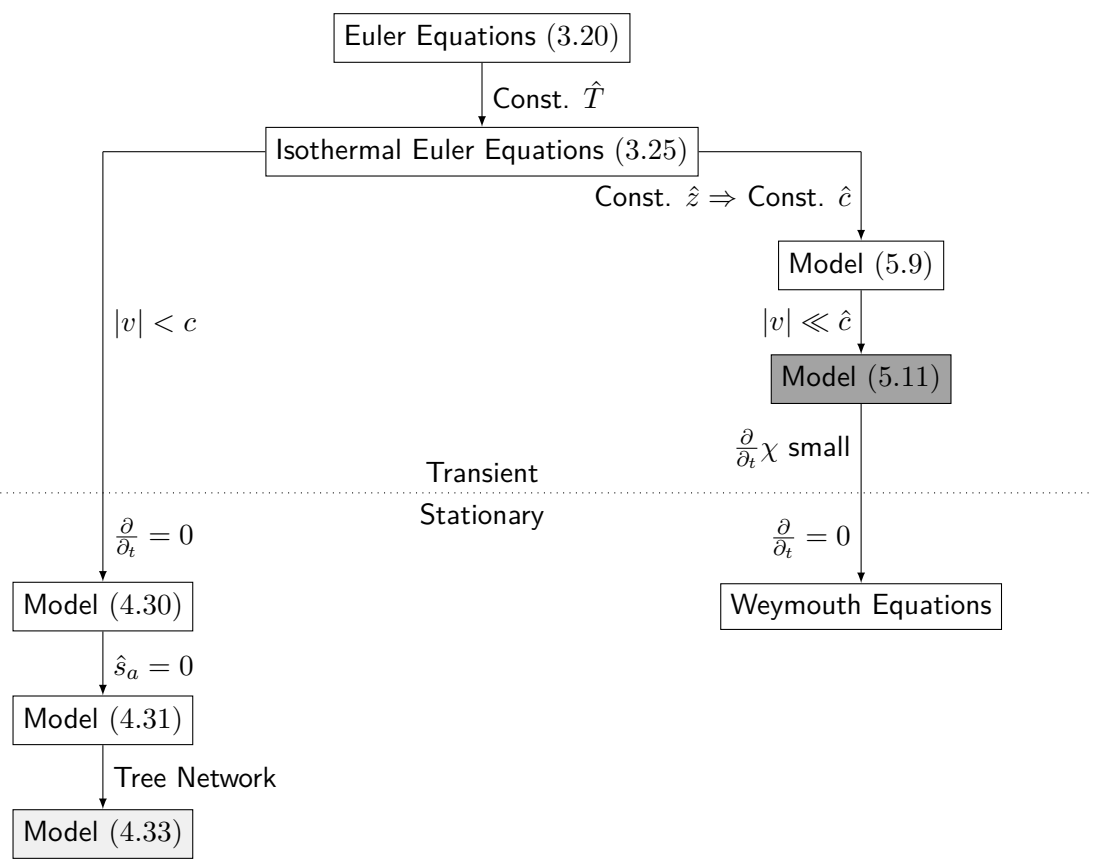

Figure 5.1: Classification of our transient pipe model (5.11) in dark gray compared to our stationary pipe model (4.33) of Chapter 4 in light gray.

within our instantaneous control approach. First, we apply an implicit Euler discretization in time that is a standard approach for gas transport problems; see, e.g., Steinbach [151]. We characterize solutions of the resulting semi-discretized system and obtain nonlinear pipe constraints eventually. Thus, Problem (5.2) is an MINLP. Second, we use the first discretization to specify a non-standard combination of explicit and implicit Euler discretizations in time that yields linear pipe constraints for every pipe within our instantaneous control approach. Consequently, Problem (5.2) is an MIP. Finally, we show a mixed implicit-explicit Euler discretization scheme of the source terms. This last discretization yields MIPs for Problem (5.2) again and is an intermediate case of the first two. These three approaches are visualized in Figure 5.2.

In (5.11), pressure $p$ and mass flux $\chi$ are functions depending on the spatial coordinate and the time. We remark that after a time discretization, pressure functions $p^{t}=p^{t}(x) \in \mathbb{R}_{>0}$ and mass flux functions $\chi^{t}=\chi^{t}(x) \in \mathbb{R}$ for all $t \in \mathcal{T}$ are obtained. Moreover, we use time steps $\Delta t_{\kappa}:=t_{\kappa+1}-t_{\kappa}$ for all $\kappa \in[K-1]$. 


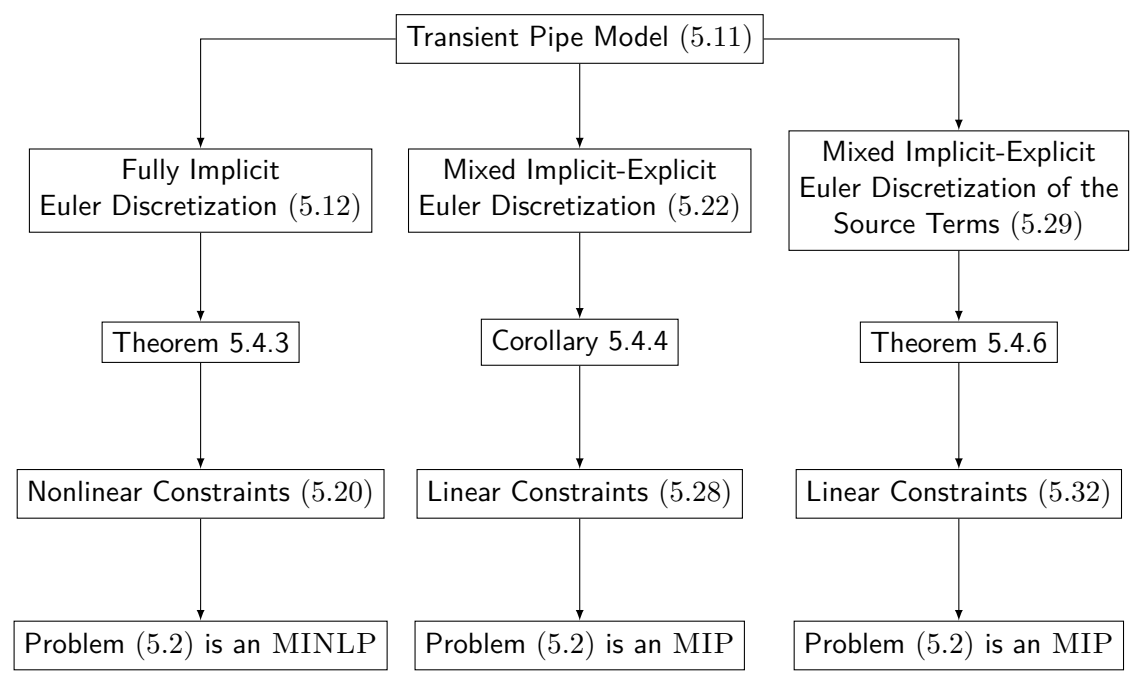

Figure 5.2: Overview of the time discretizations for (5.11).

Fully Implicit Euler Discretization We start with the transient pipe model (5.11). An implicit Euler discretization in time yields

$$
\begin{aligned}
\frac{p^{t_{\kappa+1}}-p^{t_{\kappa}}}{\Delta t_{\kappa}}+\hat{c}^{2} \partial_{x} \chi^{t_{\kappa+1}} & =0, \\
\frac{\chi^{t_{\kappa+1}}-\chi^{t_{\kappa}}}{\Delta t_{\kappa}}+\partial_{x} p^{t_{\kappa+1}} & =-\frac{\hat{\theta}_{a} \hat{c}^{2}}{2} \frac{\chi^{t_{\kappa+1}}\left|\chi^{t_{\kappa+1}}\right|}{p^{t_{\kappa+1}}}-\frac{\hat{g} \hat{s}_{a}}{\hat{c}^{2}} p^{t_{\kappa+1}} .
\end{aligned}
$$

In vector notation, we can write

$$
\begin{aligned}
\partial_{x}\left(\begin{array}{l}
\chi^{t_{\kappa+1}} \\
p^{t_{\kappa+1}}
\end{array}\right)+ & {\left[\begin{array}{cc}
0 & \frac{1}{\hat{c}^{2} \Delta t_{\kappa}} \\
\frac{1}{\Delta t_{\kappa}} & 0
\end{array}\right]\left(\begin{array}{l}
\chi^{t_{\kappa+1}} \\
p^{t_{\kappa+1}}
\end{array}\right) } \\
& =\left(\begin{array}{c}
\frac{p^{t_{\kappa}}}{\hat{c}^{2} \Delta t_{\kappa}} \\
-\frac{\hat{\theta}_{a} \hat{c}^{2}}{2} \frac{\chi^{t_{\kappa+1}}\left|\chi^{t_{\kappa+1}}\right|}{p^{t_{\kappa+1}}}-\frac{\hat{g} \hat{s}_{a}}{\hat{c}^{2}} p^{t_{\kappa+1}}+\frac{\chi^{t_{\kappa}}}{\Delta t_{\kappa}}
\end{array}\right)
\end{aligned}
$$

under the initial conditions

$$
\left(\begin{array}{c}
\chi^{t_{\kappa+1}}(0) \\
p^{t_{\kappa+1}}(0)
\end{array}\right)=y_{0} \in \mathbb{R} \times \mathbb{R}_{>0}
$$

for (5.12). 
Theorem 5.4.3. Let

$$
\begin{aligned}
\xi(x) & =\frac{1}{2} \exp \left(\frac{x}{\hat{c} \Delta t_{\kappa}}\right), \\
h(x) & =\frac{p^{t_{\kappa}}(x)}{\hat{c}^{2} \Delta t_{\kappa}}
\end{aligned}
$$

and

$$
\begin{aligned}
\zeta\left(x, \chi^{t_{\kappa+1}}, p^{t_{\kappa+1}}\right)= & -\frac{\hat{\theta}_{a} \hat{c}^{2}}{2} \frac{\chi^{t_{\kappa+1}}(x)\left|\chi^{t_{\kappa+1}}(x)\right|}{p^{t_{\kappa+1}}(x)} \\
& -\frac{\hat{g} \hat{s}_{a}}{\hat{c}^{2}} p^{t_{\kappa+1}}(x)+\frac{\chi^{t_{\kappa}}(x)}{\Delta t_{\kappa}} .
\end{aligned}
$$

Then the solution of System (5.13) under the initial conditions (5.14) satisfies

$$
\begin{aligned}
\chi^{t_{\kappa+1}}(x)= & (\xi(x)+\xi(-x)) \chi^{t_{\kappa+1}}(0) \\
& +\left(-\frac{1}{\hat{c}} \xi(x)+\frac{1}{\hat{c}} \xi(-x)\right) p^{t_{\kappa+1}}(0) \\
& +I_{\chi}\left(x, \chi^{t_{\kappa+1}}, p^{t_{\kappa+1}}\right)
\end{aligned}
$$

and

$$
\begin{aligned}
p^{t_{\kappa+1}}(x)= & (-\hat{c} \xi(x)+\hat{c} \xi(-x)) \chi^{t_{\kappa+1}}(0)+(\xi(x)+\xi(-x)) p^{t_{\kappa+1}}(0) \\
& +I_{p}\left(x, \chi^{t_{\kappa+1}}, p^{t_{\kappa+1}}\right)
\end{aligned}
$$

where we use

$$
\begin{aligned}
I_{\chi}\left(x, \chi^{t_{\kappa+1}}, p^{t_{\kappa+1}}\right) & =I_{\chi}^{h}(x)+I_{\chi}^{\zeta}\left(x, \chi^{t_{\kappa+1}}, p^{t_{\kappa+1}}\right), \\
I_{p}\left(x, \chi^{t_{\kappa+1}}, p^{t_{\kappa+1}}\right) & =I_{p}^{h}(x)+I_{p}^{\zeta}\left(x, \chi^{t_{\kappa+1}}, p^{t_{\kappa+1}}\right),
\end{aligned}
$$

and

$$
\begin{aligned}
I_{\chi}^{h}(x) & =\int_{0}^{x}(\xi(x-s)+\xi(-(x-s))) h(s) \mathrm{d} s \\
I_{\chi}^{\zeta}\left(x, \chi^{t_{\kappa+1}}, p^{t_{\kappa+1}}\right) & =\int_{0}^{x}\left(-\frac{1}{\hat{c}} \xi(x-s)+\frac{1}{\hat{c}} \xi(-(x-s))\right) \zeta\left(s, \chi^{t_{\kappa+1}}, p^{t_{\kappa+1}}\right) \mathrm{d} s, \\
I_{p}^{h}(x) & =\int_{0}^{x}(-\hat{c} \xi(x-s)+\hat{c} \xi(-(x-s))) h(s) \mathrm{d} s \\
I_{p}^{\zeta}\left(x, \chi^{t_{\kappa+1}}, p^{t_{\kappa+1}}\right) & =\int_{0}^{x}(\xi(x-s)+\xi(-(x-s))) \zeta\left(s, \chi^{t_{\kappa+1}}, p^{t_{\kappa+1}}\right) \mathrm{d} s .
\end{aligned}
$$


Proof. Using the abbreviations

$$
y:=\left(\begin{array}{c}
\chi^{t_{\kappa+1}} \\
p^{t_{\kappa+1}}
\end{array}\right), \quad B:=\left[\begin{array}{cc}
0 & \frac{1}{\hat{c}^{2} \Delta t_{\kappa}} \\
\frac{1}{\Delta t_{\kappa}} & 0
\end{array}\right]
$$

we see that System (5.13) is equivalent to

$$
\partial_{x} y(x)+B y(x)=\left(\begin{array}{c}
h(x) \\
\zeta\left(x, \chi^{t_{\kappa+1}}, p^{t_{\kappa+1}}\right)
\end{array}\right) .
$$

A solution is given by

$$
y(x)=\exp (-B x) y_{0}+\int_{0}^{x} \exp (-B(x-s))\left(\begin{array}{c}
h(s) \\
\zeta\left(s, \chi^{t_{\kappa+1}}, p^{t_{\kappa+1}}\right)
\end{array}\right) \mathrm{d} s
$$

where $y_{0}=\left(\chi^{t_{\kappa+1}}(0), p^{t_{\kappa+1}}(0)\right)^{\top}$ consists of the mass flux and pressure values at the beginning of the pipe, i.e., at $x=0$; see (5.14). This follows from Duhamel's formula; see, e.g., Coddington and Carlson [31, Theorem 3.4]. Diagonalization of $-B$ yields

$$
-B=S D S^{-1}
$$

with

$$
S=\left[\begin{array}{cc}
-\frac{1}{\hat{c}} & \frac{1}{\hat{c}} \\
1 & 1
\end{array}\right], \quad D=\left[\begin{array}{cc}
\frac{1}{\hat{c} \Delta t_{\kappa}} & 0 \\
0 & -\frac{1}{\hat{c} \Delta t_{\kappa}}
\end{array}\right], \quad S^{-1}=\left[\begin{array}{cc}
-\frac{\hat{c}}{2} & \frac{1}{2} \\
\frac{\hat{c}}{2} & \frac{1}{2}
\end{array}\right],
$$

and we thus have

$$
\begin{aligned}
\exp (-B x) & =\left[\begin{array}{cc}
-\frac{1}{\hat{c}} & \frac{1}{\hat{c}} \\
1 & 1
\end{array}\right]\left[\begin{array}{cc}
\exp \left(\frac{x}{\hat{c} \Delta t_{\kappa}}\right) & 0 \\
0 & \exp \left(-\frac{x}{\hat{c} \Delta t_{\kappa}}\right)
\end{array}\right]\left[\begin{array}{cc}
-\frac{\hat{c}}{2} & \frac{1}{2} \\
\frac{\hat{c}}{2} & \frac{1}{2}
\end{array}\right] \\
& =\left[\begin{array}{cc}
\xi(x)+\xi(-x) & -\frac{1}{\hat{c}} \xi(x)+\frac{1}{\hat{c}} \xi(-x) \\
-\hat{c} \xi(x)+\hat{c} \xi(-x) & \xi(x)+\xi(-x)
\end{array}\right] .
\end{aligned}
$$

Analogously, we get

$$
\begin{aligned}
& \exp (-B(x-s))= \\
& {\left[\begin{array}{cc}
\xi(x-s)+\xi(-(x-s)) & -\frac{1}{\hat{c}} \xi(x-s)+\frac{1}{\hat{c}} \xi(-(x-s)) \\
-\hat{c} \xi(x-s)+\hat{c} \xi(-(x-s)) & \xi(x-s)+\xi(-(x-s))
\end{array}\right] .}
\end{aligned}
$$

Substituting both matrix exponentials into (5.18) finally yields the claim. 
We use a spatial discretization that couples the equations in (5.16) and (5.17) with the variables $p_{u}^{t}, p_{w}^{t}, \chi_{a, u}^{t}$, and $\chi_{a, w}^{t}$. Consequently,

$$
\begin{aligned}
p_{u}^{t}=p(0, t), p_{w}^{t}=p\left(\hat{L}_{a}, t\right) & \text { for all } a=(u, w) \in A_{\mathrm{pi}}, t \in \mathcal{T}, \\
\chi_{a, u}^{t}=\chi(0, t), \chi_{a, w}^{t}=\chi\left(\hat{L}_{a}, t\right) & \text { for all } a=(u, w) \in A_{\mathrm{pi}}, t \in \mathcal{T},
\end{aligned}
$$

can be obtained. We can approximate the integrals using numerical integration schemes like the trapezodial rule and write

$$
\begin{aligned}
\widetilde{I}_{\chi}\left(\hat{L}_{a}, \chi_{a, u}^{t_{\kappa+1}}, \chi_{a, w}^{t_{\kappa+1}}, p_{u}^{t_{\kappa+1}}, p_{w}^{t_{\kappa+1}}\right) & \approx I_{\chi}\left(\hat{L}_{a}, \chi^{t_{\kappa+1}}, p^{t_{\kappa+1}}\right), \\
\widetilde{I}_{p}\left(\hat{L}_{a}, \chi_{a, u}^{t_{\kappa+1}}, \chi_{a, w}^{t_{\kappa+1}}, p_{u}^{t_{\kappa+1}}, p_{w}^{t_{\kappa+1}}\right) & \approx I_{p}\left(\hat{L}_{a}, \chi^{t_{\kappa+1}}, p^{t_{\kappa+1}}\right) .
\end{aligned}
$$

Eventually, we derive the constraints

$$
\begin{aligned}
\left(\begin{array}{c}
\chi_{a, w}^{t_{\kappa+1}} \\
p_{w}^{t_{\kappa+1}}
\end{array}\right)= & {\left[\begin{array}{cc}
\xi\left(\hat{L}_{a}\right)+\xi\left(-\hat{L}_{a}\right) & -\frac{1}{\hat{c}} \xi\left(\hat{L}_{a}\right)+\frac{1}{\hat{c}} \xi\left(-\hat{L}_{a}\right) \\
-\hat{c} \xi\left(\hat{L}_{a}\right)+\hat{c} \xi\left(-\hat{L}_{a}\right) & \xi\left(\hat{L}_{a}\right)+\xi\left(-\hat{L}_{a}\right)
\end{array}\right]\left(\begin{array}{c}
\chi_{a, u}^{t_{\kappa+1}} \\
p_{u}^{t_{\kappa+1}}
\end{array}\right) } \\
& +\left(\begin{array}{l}
\widetilde{I}_{\chi}\left(\hat{L}_{a}, \chi_{a, u}^{t_{\kappa+1}}, \chi_{a, w}^{t_{\kappa+1}}, p_{u}^{t_{\kappa+1}}, p_{w}^{t_{\kappa+1}}\right) \\
\widetilde{I}_{p}\left(\hat{L}_{a}, \chi_{a, u}^{t_{\kappa+1}}, \chi_{a, w}^{t_{k+1}}, p_{u}^{t_{\kappa+1}}, p_{w}^{t_{\kappa+1}}\right)
\end{array}\right)
\end{aligned}
$$

for every pipe $a=(u, w) \in A_{\mathrm{pi}}$. These nonlinear constraints couple the mass flux and the pressure variables in time step $t_{\kappa+1}$. The nonlinearity only appears due to $\widetilde{I}_{\chi}$ and $\widetilde{I}_{p}$. Note that it remains independent of whether we use an analytical formula for the antiderivatives of the integrals $I_{\chi}$ and $I_{p}$ or whether we use numerical integration schemes like the trapezoidal rule. The latter still implies nonlinearities in the evaluation of the inhomogeneity $\zeta$; see (5.15).

Thus, in the case of a fully implicit Euler discretization, Problem (5.2) is an MINLP for time step $t_{\kappa+1}$ with $x_{\mathcal{C}}^{t_{\kappa+1}} \in \mathcal{X}^{t_{\kappa+1}}$ defined by (5.20); see Figure 5.2.

Mixed Implicit-Explicit Euler Discretization As in the last section, we start with the transient pipe model (5.11). Guided by the results of the last section, we now reduce the resulting nonlinearity in the right-hand side of the semi-discretized system. To this end, we consider the case in which we replace the inhomogeneity $\zeta\left(x, \chi^{t_{\kappa+1}}, p^{t_{\kappa+1}}\right)$ in (5.15) with $\zeta(x)$ given as

$$
\zeta(x):=-\frac{\hat{\theta}_{a} \hat{c}^{2}}{2} \frac{\chi^{t_{\kappa}}(x)\left|\chi^{t_{\kappa}}(x)\right|}{p^{t_{\kappa}}(x)}-\frac{\hat{g} \hat{s}_{a}}{\hat{c}^{2}} p^{t_{\kappa}}(x)+\frac{\chi^{t_{\kappa}}(x)}{\Delta t_{\kappa}} .
$$


This means that we apply an explicit Euler discretization to the right-hand side of the momentum equation in (5.11b). Taken all together, this mixed implicit-explicit Euler discretization yields

$$
\begin{aligned}
\frac{p^{t_{\kappa+1}}-p^{t_{\kappa}}}{\Delta t_{\kappa}}+\hat{c}^{2} \partial_{x} \chi^{t_{\kappa+1}} & =0, \\
\frac{\chi^{t_{\kappa+1}}-\chi^{t_{\kappa}}}{\Delta t_{\kappa}}+\partial_{x} p^{t_{\kappa+1}} & =-\frac{\hat{\theta}_{a} \hat{c}^{2}}{2} \frac{\chi^{t_{\kappa}}\left|\chi^{t_{\kappa}}\right|}{p^{t_{\kappa}}}-\frac{\hat{g} \hat{s}_{a}}{\hat{c}^{2}} p^{t_{\kappa}} .
\end{aligned}
$$

In vector notation, we can write

$$
\begin{aligned}
& \partial_{x}\left(\begin{array}{l}
\chi^{t_{\kappa+1}} \\
p^{t_{\kappa+1}}
\end{array}\right)+\left[\begin{array}{cc}
0 & \frac{1}{\hat{c}^{2} \Delta t_{\kappa}} \\
\frac{1}{\Delta t_{\kappa}} & 0
\end{array}\right]\left(\begin{array}{l}
\chi^{t_{\kappa+1}} \\
p^{t_{\kappa+1}}
\end{array}\right) \\
& =\left(\begin{array}{c}
\frac{p^{t_{\kappa}}}{\hat{c}^{2} \Delta t_{\kappa}} \\
-\frac{\hat{\theta}_{a} \hat{c}^{2}}{2} \frac{\chi^{t_{\kappa}}\left|\chi^{t_{\kappa}}\right|}{p^{t_{\kappa}}}-\frac{\hat{g} \hat{s}_{a}}{\hat{c}^{2}} p^{t_{\kappa}}+\frac{\chi^{t_{\kappa}}}{\Delta t_{\kappa}}
\end{array}\right)
\end{aligned}
$$

under the initial conditions

$$
\left(\begin{array}{l}
\chi^{t_{\kappa+1}}(0) \\
p^{t_{\kappa+1}}(0)
\end{array}\right)=y_{0} \in \mathbb{R} \times \mathbb{R}_{>0}
$$

for (5.22). Applying the same arguments as before yields the following result:

Corollary 5.4.4. Let $\xi, h, I_{\chi}^{h}$, and $I_{p}^{h}$ be given as in Theorem 5.4.3 and $\zeta$ be given as in (5.21). Then the solution of System (5.23) under the initial conditions (5.24) satisfies

$$
\begin{aligned}
\chi^{t_{\kappa+1}}(x)= & (\xi(x)+\xi(-x)) \chi^{t_{\kappa+1}}(0) \\
& +\left(-\frac{1}{\hat{c}} \xi(x)+\frac{1}{\hat{c}} \xi(-x)\right) p^{t_{\kappa+1}}(0)+\stackrel{\circ}{I}_{\chi}(x)
\end{aligned}
$$

and

$$
\begin{aligned}
p^{t_{\kappa+1}}(x)= & (-\hat{c} \xi(x)+\hat{c} \xi(-x)) \chi^{t_{\kappa+1}}(0) \\
& +(\xi(x)+\xi(-x)) p^{t_{\kappa+1}}(0)+\stackrel{\circ}{I}_{p}(x)
\end{aligned}
$$

where we use

$$
\begin{gathered}
\stackrel{\circ}{I}_{\chi}(x)=I_{\chi}^{h}(x)+\stackrel{\circ}{I}_{\chi}^{\zeta}(x), \\
\stackrel{\circ}{I}_{p}(x)=I_{p}^{h}(x)+\stackrel{\circ}{I_{p}^{\zeta}}(x),
\end{gathered}
$$


and

$$
\begin{aligned}
& \stackrel{\circ}{I_{\chi}^{\zeta}}(x)=\int_{0}^{x}\left(-\frac{1}{\hat{c}} \xi(x-s)+\frac{1}{\hat{c}} \xi(-(x-s))\right) \zeta(s) \mathrm{d} s, \\
& \stackrel{\circ}{I_{p}^{\zeta}}(x)=\int_{0}^{x}(\xi(x-s)+\xi(-(x-s))) \zeta(s) \mathrm{d} s .
\end{aligned}
$$

Proof. Follows directly from Theorem 5.4.3.

We use the same spatial discretization as for the fully implicit Euler discretization; see (5.19). Corollary 5.4.4 now reveals the fact that an explicit discretization of the source terms returns a linear connection between $p_{u}^{t_{\kappa+1}}$, $p_{w}^{t_{\kappa+1}}, \chi_{a, u}^{t_{\kappa+1}}$, and $\chi_{a, w}^{t_{\kappa+1}}$. Furthermore, we can approximate the integrals using numerical integration schemes like the trapezodial rule again and write

$$
\begin{aligned}
\widetilde{I}_{\chi}\left(\hat{L}_{a}\right) & \approx \stackrel{\circ}{I}_{\chi}\left(\hat{L}_{a}\right), \\
\widetilde{I}_{p}\left(\hat{L}_{a}\right) & \approx \stackrel{\circ}{I}_{p}\left(\hat{L}_{a}\right) .
\end{aligned}
$$

Eventually, (5.25) and (5.26) yield the constraints

$$
\begin{aligned}
\left(\begin{array}{c}
\chi_{a, w}^{t_{\kappa+1}} \\
p_{w}^{t_{\kappa+1}}
\end{array}\right)= & {\left[\begin{array}{cc}
\xi\left(\hat{L}_{a}\right)+\xi\left(-\hat{L}_{a}\right) & -\frac{1}{\hat{c}} \xi\left(\hat{L}_{a}\right)+\frac{1}{\hat{c}} \xi\left(-\hat{L}_{a}\right) \\
-\hat{c} \xi\left(\hat{L}_{a}\right)+\hat{c} \xi\left(-\hat{L}_{a}\right) & \xi\left(\hat{L}_{a}\right)+\xi\left(-\hat{L}_{a}\right)
\end{array}\right]\left(\begin{array}{c}
\chi_{a, u}^{t_{\kappa+1}} \\
p_{u}^{t_{\kappa+1}}
\end{array}\right) } \\
& +\left(\begin{array}{c}
\widetilde{I}_{\chi}\left(\hat{L}_{a}\right) \\
\widetilde{I}_{p}\left(\hat{L}_{a}\right)
\end{array}\right)
\end{aligned}
$$

for every pipe $a=(u, w) \in A_{\mathrm{pi}}$. The linearity property holds because the integrals $\widetilde{I}_{\chi}\left(\hat{L}_{a}\right)$ and $\widetilde{I}_{p}\left(\hat{L}_{a}\right)$ only depend on the solution of the previous time step, i.e., on $p_{u}^{t_{\kappa}}, p_{w}^{t_{\kappa}}, \chi_{a, u}^{t_{\kappa}}$, and $\chi_{a, w}^{t_{\kappa}}$. These quantities are known as a result of the problem solved in the last step of the instantaneous control approach and the integrals can be approximated by numerical integration.

Thus, in the case of a mixed implicit-explicit Euler discretization, Problem (5.2) is an MIP for time step $t_{\kappa+1}$ with $x_{\mathcal{C}}^{t_{\kappa+1}} \in \mathcal{X}^{t_{\kappa+1}}$ defined by (5.28); see Figure 5.2.

Remark 5.4.5 (Existence and Uniqueness). We state a well-posedness result in our article Gugat et al. [76]. This result was elaborated by Prof. Dr. Günter Leugering, Prof. Dr. Martin Schmidt, and David Wintergerst. 


\section{Mixed Implicit-Explicit Euler Discretization of the Source Terms}

As in the last two sections, we start with the transient pipe model (5.11). Regarding the two last time discretization schemes, i.e., the fully implicit and the mixed implicit-explicit scheme, one may also think of a further intermediate case. We now show how this intermediate case can be formulated to maintain linear formulations. To this end, we apply a mixed explicit-implicit discretization of the source term of the momentum equation and obtain

$$
\begin{aligned}
\frac{p^{t_{\kappa+1}}-p^{t_{\kappa}}}{\Delta t_{\kappa}}+\hat{c}^{2} \partial_{x} \chi^{t_{\kappa+1}} & =0, \\
\frac{\chi^{t_{\kappa+1}}-\chi^{t_{\kappa}}}{\Delta t_{\kappa}}+\partial_{x} p^{t_{\kappa+1}} & =-\frac{\hat{\theta}_{a} \hat{c}^{2}}{2} \frac{\left|\chi^{t_{\kappa}}\right| \chi^{t_{\kappa+1}}}{p^{t_{\kappa}}}-\frac{\hat{g} \hat{s}_{a}}{\hat{c}^{2}} p^{t_{\kappa}} .
\end{aligned}
$$

In vector notation, we can write

$$
\begin{array}{r}
\partial_{x}\left(\begin{array}{c}
\chi^{t_{\kappa+1}} \\
p^{t_{\kappa+1}}
\end{array}\right)+\left[\begin{array}{cc}
0 & \frac{1}{\hat{c}^{2} \Delta t_{\kappa}} \\
\frac{1}{\Delta t_{\kappa}}+\frac{\hat{c}^{2} \hat{\theta}_{a}}{2} \frac{\left|\chi^{t_{\kappa}}\right|}{p^{t_{\kappa}}} & 0
\end{array}\right]\left(\begin{array}{c}
\chi^{t_{\kappa+1}} \\
p^{t_{\kappa+1}}
\end{array}\right) \\
=\left(\begin{array}{c}
\frac{p^{t_{\kappa}}}{\hat{c}^{2} \Delta t_{\kappa}} \\
-\frac{\hat{g} \hat{s}_{a}}{\hat{c}^{2}} p^{t_{\kappa}}+\frac{\chi^{t_{\kappa}}}{\Delta t_{\kappa}}
\end{array}\right)
\end{array}
$$

under the initial conditions

$$
\left(\begin{array}{l}
\chi^{t_{\kappa+1}}(0) \\
p^{t_{\kappa+1}}(0)
\end{array}\right)=y_{0} \in \mathbb{R} \times \mathbb{R}_{>0}
$$

for (5.29). The main difference with respect to System (5.13) is the dependence of the system matrix on $x$ due to the appearing functions $p^{t_{\kappa}}$ and $\chi^{t_{\kappa}}$. The solution can be formulated with the help of a matrix exponential.

Theorem 5.4.6. Let $h$ be given as in Theorem 5.4.3. Moreover, let

$$
M(x)=\left[\begin{array}{cc}
0 & \frac{1}{\hat{c}^{2} \Delta t_{\kappa}} \\
\frac{1}{\Delta t_{\kappa}}+\frac{\hat{c}^{2} \hat{\theta}_{a}}{2} \frac{\left|\chi^{t_{\kappa}}(x)\right|}{p^{t_{\kappa}}(x)} & 0
\end{array}\right]
$$

and

$$
l(x)=-\frac{\hat{g} \hat{s}_{a}}{\hat{c}^{2}} p^{t_{\kappa}}(x)+\frac{\chi^{t_{\kappa}}(x)}{\Delta t_{\kappa}} .
$$


Then the solution of System (5.30) under the initial conditions (5.31) satisfies

$$
\left(\begin{array}{l}
\chi^{t_{\kappa+1}}(x) \\
p^{t_{\kappa+1}}(x)
\end{array}\right)=\exp (F(x))\left(y_{0}+\int_{0}^{x} \exp (-F(s))\left(\begin{array}{c}
h(s) \\
l(s)
\end{array}\right) \mathrm{d} s\right)
$$

where we use

$$
F(x)=\int_{0}^{x}-M(s) \mathrm{d} s .
$$

Proof. This follows from variation of parameters.

In Theorem 5.4.3, we are able to transform the matrix exponential for the fully implicit Euler discretization by diagonalization. In contrast to that, the idea here is to use numerical integration directly to obtain a linear constraint set. We apply the spatial discretization (5.19) again and approximate $F\left(\hat{L}_{a}\right)$ and the inner integral using the trapezoidal rule again, i.e.,

$$
\begin{aligned}
I_{1}\left(\hat{L}_{a}\right) & :=\exp \left(F\left(\hat{L}_{a}\right)\right) \\
& \approx \exp \left(-\frac{\hat{L}_{a}}{2}\left(M(0)+M\left(\hat{L}_{a}\right)\right)\right) \\
& =: \widetilde{I}_{1}\left(\hat{L}_{a}\right)
\end{aligned}
$$

and

$$
\begin{aligned}
I_{2}\left(\hat{L}_{a}\right) & :=\int_{0}^{\hat{L}_{a}} \exp (-F(s))\left(\begin{array}{c}
h(s) \\
l(s)
\end{array}\right) \mathrm{d} s \\
& \approx \frac{\hat{L}_{a}}{2}\left(\exp \left(-F\left(\hat{L}_{a}\right)\right)\left(\begin{array}{c}
h\left(\hat{L}_{a}\right) \\
l\left(\hat{L}_{a}\right)
\end{array}\right)+\exp (-F(0))\left(\begin{array}{c}
h(0) \\
l(0)
\end{array}\right)\right) \\
& =: \widetilde{I}_{2}\left(\hat{L}_{a}\right) .
\end{aligned}
$$

We obtain that $\widetilde{I}_{1}\left(\hat{L}_{a}\right)$ and $\widetilde{I}_{2}\left(\hat{L}_{a}\right)$ only depend on the solution of the previous time step, i.e., on $p_{u}^{t_{\kappa}}, p_{w}^{t_{\kappa}}, \chi_{a, u}^{t_{\kappa}}$, and $\chi_{a, w}^{t_{\kappa}}$. Nevertheless, we remark that the calculation of $\widetilde{I}_{1}\left(\hat{L}_{a}\right)$ and $\widetilde{I}_{2}\left(\hat{L}_{a}\right)$ involves matrix exponentials. Finally, this yields the linear constraints

$$
\left(\begin{array}{c}
\chi_{a, w}^{t_{\kappa+1}} \\
p_{w}^{t_{\kappa+1}}
\end{array}\right)=\widetilde{I}_{1}\left(\hat{L}_{a}\right)\left(\begin{array}{c}
\chi_{a, u}^{t_{\kappa+1}} \\
p_{u}^{t_{\kappa+1}}
\end{array}\right)+\widetilde{I}_{1}\left(\hat{L}_{a}\right) \widetilde{I}_{2}\left(\hat{L}_{a}\right)
$$

for every pipe $a=(u, w) \in A_{\text {pi }}$.

Thus, in the case of a mixed implicit-explicit Euler discretization of the source terms, Problem (5.2) is an MIP for time step $t_{\kappa+1}$ with $x_{\mathcal{C}}^{t_{\kappa+1}} \in \mathcal{X}^{t_{\kappa+1}}$ defined by (5.32); see Figure 5.2. 


\subsubsection{Numerical Results}

The following results are obtained with the constraints derived from the mixed implicit-explicit time discretization; see (5.28). Moreover, we use the trapezoidal rule to calculate the integrals $\widetilde{I}_{\chi}$ and $\widetilde{I}_{p}$; see (5.27). Thus, we solve MIPs for Problem (5.2); see Figure 5.2. Our transient gas transport optimization problem reads

min LP-modeled (5.3)

s.t. pressure variables: $(3.1)$;

mass flow variables: (3.2), (3.3);

mass flux variables: (3.4);

power variables: $(3.5)$;

(TransGas)

mass flow/mass flux connection: (3.26);

mass flow conservation: (3.6);

valve, control valve, compr.: linearized (3.7)-(3.19);

pipe model: (5.28).

Problem (TransGas) is an example for Problem (5.1) and we can use Algorithm 5.1. We consider case studies to illustrate the applicability of our instantaneous control approach to realistic control problems.

Our first testset is a minimal example with a single pipe to analyze the physical phenomenon of pressure and flow waves. This is a litmus test to check if our pipe model (5.28) yields plausible physical solutions. Afterward, we investigate a bigger network with all elements from Chapter 3. For both testsets, we introduce the data first and show promising numerical results afterward. Our instantaneous control approach and all models are implemented using the $\mathrm{C}++$ framework LaMaTTO ++ ; see LaMaTTO $++[103]$. All MIP models are solved with Gurobi 6.5.0 using all the available 4 threads; see Gurobi [80]. The data analysis of the results was performed by Python Pandas 0.20.3 inside of Python 3.5.2; see McKinney [119]. All computations were performed on an $\operatorname{Intel}^{\odot} \mathrm{Core}^{\mathrm{TM}} \mathrm{i} 5-3360 \mathrm{M}$ CPU with 4 cores and $2.8 \mathrm{GHz}$ each and 4 GB RAM.

\section{Testset One}

We describe the data and state results and remarks for Algorithm 5.1.

Data We consider a minimal example of a fictional single pipe as in Figure 5.3 and describe the main properties. 


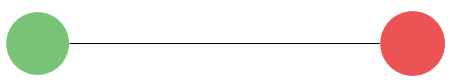

Figure 5.3: Network of Testset One with 1 entry node (green), 1 exit node (red), and 1 pipe (black).

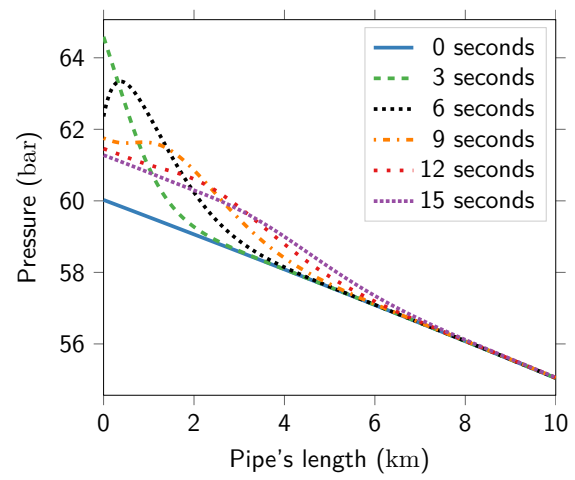

(a) Pressure profile.

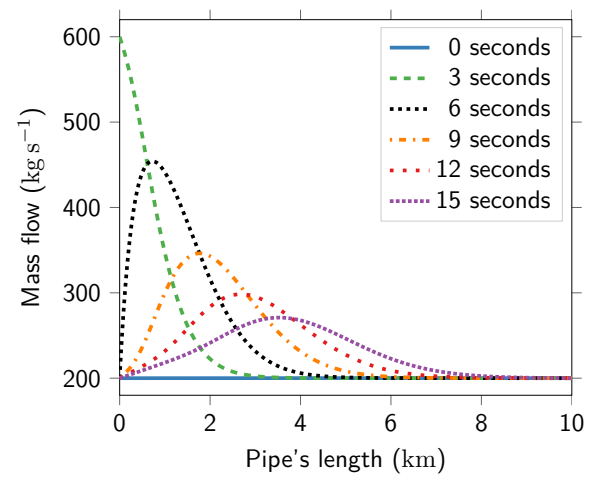

(b) Mass flow profile.

Figure 5.4: Profiles over the pipe's length for different time points.

We use a pipe with the length of $10 \mathrm{~km}$, the diameter of $0.7 \mathrm{~m}$, and the roughness of $0.1 \mathrm{~mm}$. On the one hand, $200 \mathrm{~kg} \mathrm{~s}^{-1}$ are constantly discharged at the pipe's exit. On the other hand, during second $0,200 \mathrm{~kg} \mathrm{~s}^{-1}$ are supplied at the pipe's entry. Then the amount is increased to $600 \mathrm{~kg} \mathrm{~s}^{-1}$ between second 1 and second 5 . Finally, from second 6 onward, the supply is again reduced to $200 \mathrm{~kg} \mathrm{~s}^{-1}$. We choose a time discretization of $1 \mathrm{~s}$ and a spatial discretization of $5 \mathrm{~m}$.

The exact data is available online. ${ }^{1}$ Three $x m l-F i l e s$ are given. The net-file describes the network and its parameters, the initial-file describes the initial conditions, and the bconditions-file specifies the development of supply and demand. The $\mathrm{xml}$-schemas are inspired by the schemas in our article Schmidt et al. [140], which is tailored for stationary gas transport problems.

We remark that constants such as gravity, speed of sound, and specific gas constant are not given in the data. Gravity $\hat{g}$ can be neglected as no height differences occur in the data. We set the speed of sound $\hat{c}=340.29 \mathrm{~m} \mathrm{~s}^{-1}$

\footnotetext{
$1 \overline{\text { https://drive.google.com/file/d/1 }} \mathrm{dgLkXG-FqOozSrtL111KuqlKox0B3Ssz/view?usp=}$ sharing
} 


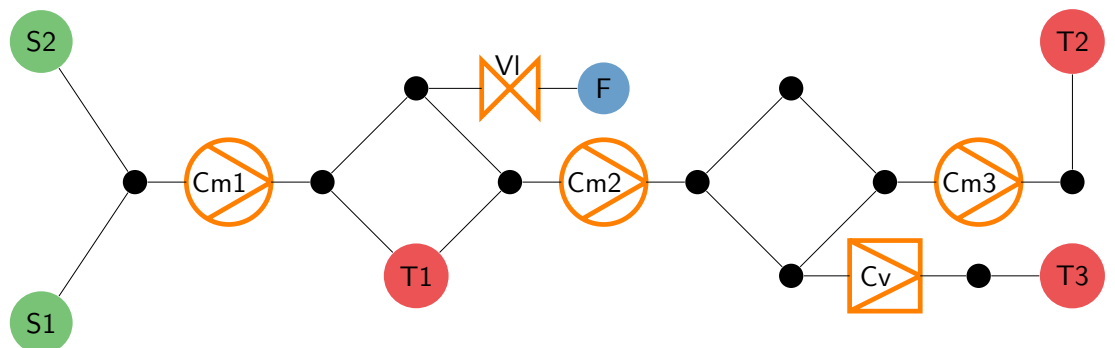

Figure 5.5: Network of Testset Two with 2 entry nodes (green), 3 exit nodes (red), 1 flexible node (blue), 10 inner nodes (black), 1 valve (orange symbol; VI), 1 control valve (orange symbol; Cv), 3 compressor machines (orange symbol; Cm1, Cm2, Cm3), and 12 pipes (black).

and the specific gas constant $\hat{R}_{\mathrm{S}}=500.27 \mathrm{~m}^{2} \mathrm{~s}^{-2} \mathrm{~K}^{-1}$. The former is needed in the pipe model, whereas the latter is used in the compressor model.

Results and Remarks for Algorithm 5.1 We remark that Problem (TransGas) only consists of pipe model (5.28) in this case. It is a feasibility problem that is solved immediately by the solver. Thus, we do not have to specify the objective function. Using the high resolutions of $1 \mathrm{~s}$ for the time discretization and $5 \mathrm{~m}$ for the spatial discretization, we observe pressure waves and mass flow waves within the pipe as shown in Figure 5.4.

Consequently, we conclude that pipe model (5.28) yields plausible physical behaviors. We provide a video with descriptions to visualize the solution. ${ }^{2}$

\section{Testset Two}

We describe the data and state results and remarks for Algorithm 5.1.

Data We consider the test network given in Figure 5.5 that is adapted from Ehrhardt and Steinbach [52]. We remark that this network, as well as the network in Figure 5.3, is a test network that does not contain geographic coordinates. We describe the main properties.

The network contains 16 nodes: 2 entries (S1, S2), 3 exits (T1, T2, T3), and one flexible node (F) that can be entry and exit over time. We remark that the consideration of flexible nodes is not completely in line with the network model presented in Chapter 3 because $u \in V_{-}$or $u \in V_{+}$for a node $u \notin V_{0}$ does not hold anymore for all time steps. However, all required

2 https://youtu.be/6F74WZ0CZ7Y 
changes in the model are straightforward. All remaining nodes (10) are inner nodes. The nodes are connected by 17 arcs that comprise 12 pipes, one valve $(\mathrm{VI})$, one control valve $(\mathrm{Cv})$, and 3 compressor machines $(\mathrm{Cm} 1, \mathrm{Cm} 2$, $\mathrm{Cm} 3$ ). The total pipe length is $92 \mathrm{~km}$ with separate pipe lengths varying between 5 and $12 \mathrm{~km}$. The pipes' diameters vary between 0.5 and $1.1 \mathrm{~m}$ and the roughness values lie between 0.01 and $0.05 \mathrm{~mm}$.

The initial state is a stationary state of the network in which all active elements are open and inactive, i.e., the valve is open, and all compressor machines and the control valve are in bypass mode. Entry S1 supplies $96 \mathrm{~kg} \mathrm{~s}^{-1}$ and entry S2 delivers $19 \mathrm{~kg} \mathrm{~s}^{-1}$. The exit customers T1 and T3 withdraw only small amounts of $2 \mathrm{~kg} \mathrm{~s}^{-1}$ and $4 \mathrm{~kg} \mathrm{~s}^{-1}$, respectively, whereas exit T2 models a large industrial customer that withdraws $108 \mathrm{~kg} \mathrm{~s}^{-1}$. As the mass flow values in the initial state are comparably small and all compressors are in bypass mode, all pressure values are around 60 bar. We choose a time discretization of $1 \mathrm{~min}$ and a spatial discretization of $100 \mathrm{~m}$.

We have to discuss the chosen objective function. We use minimization of (5.3) with $\mu_{u}>\eta_{u}$, i.e., we penalize deviations from the target mass flows harder than deviations in target pressure. More precisely, we choose $\mu_{u}=100$ and $\nu_{u}=1$ with all mass flow values given in $\mathrm{m} \mathrm{s}^{-1}$ and all pressure values given in bar. We use the $\infty$-norm, i.e., Model (5.7).

The exact data is available online. ${ }^{3}$ Two $\mathrm{xml-Files}$ are given. The net-file describes the network and its parameters, while the initial-file describes the initial conditions. In this case, the bconditions-file is needless as we use a tracking type objective that controls supplied and discharged gas as well as the corresponding pressure. Again, the xml-schema is inspired by the schemas in our article Schmidt et al. [140]. For the exact characteristic diagrams of the compressors we use the data of compressor_1 of the publicly available instance GasLib-135 for $\mathrm{Cm} 1, \mathrm{Cm} 2$, and $\mathrm{Cm} 3{ }^{4}$

Furthermore, gravity $\hat{g}$ can be neglected again, whereas the speed of sound is set to $\hat{c}=340.29 \mathrm{~m} \mathrm{~s}^{-1}$ and the specific gas constant is set to $\hat{R}_{\mathrm{s}}=500.27 \mathrm{~m}^{2} \mathrm{~s}^{-2} \mathrm{~K}^{-1}$. Once more the former is needed in the pipe model and the latter is used in the compressor model.

Results and Remarks for Algorithm 5.1 We remark that Problem (TransGas) exists in its full beauty in this case. We discuss the testcase in detail.

\footnotetext{
${ }^{3}$ https://drive.google.com/file/d/1D3neGSnkOhUUS2iSipl2rIKtXd4Y5ntf/view?usp= sharing

${ }^{4}$ http://gaslib.zib.de
} 


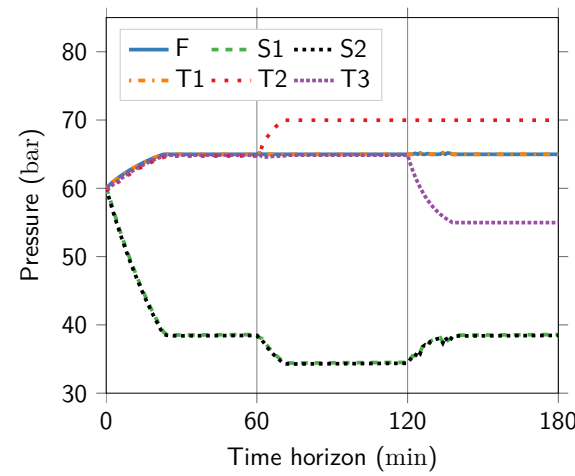

(a) Pressure profile.

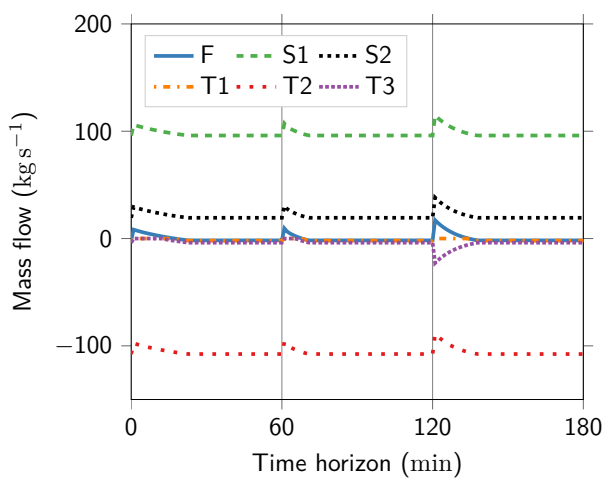

(b) Mass flow profile.

Figure 5.6: Profiles over the time horizon at entries, exists, and the flexible node.

As already mentioned in the data description of the initial state, we start with an almost equal pressure of approximately 60 bar at all nodes. We take our initial state, which is stationary, and use it for the tracking-type objective function. In this case, the network would stay stable over time.

We change the objective function to observe dynamical behavior. Note that the instantaneous control approach obviously has limitations in reaching terminal states that require an action early in the control period or that considerably differ from the initial state. This is why we split a time horizon of 3 hours into 3 blocks of 1 hour each and state a new tracking target in every control sub-period, i.e., in every hour. On the other hand, in real-time operations, this strategy may be used to address uncertainties that realize during the considered overall time horizon.

We now turn to the discussion of the results of the instantaneous control approach. The pressure and mass flow profiles of all entries and exits are given in Figure 5.6. Moreover, the pressure difference profiles for the compressor machines and the control valve are given in Figure 5.7. Again, we provide a video with descriptions to visualize the solution. ${ }^{5}$

At the beginning of the first hour, we set $\hat{p}_{\mathrm{T} 1}=65$ bar and $\nu_{\mathrm{T} 1}=1000$ to reach a higher pressure for the exit customer at T1. The high value for $\nu_{\mathrm{T} 1}$ emphasizes the control of T1. All other entry, exit, and flexible pressures, with $\nu_{u}=1$ still, may be adjusted as required within certain technical bounds that ensure the validity of our physical model. The constant penalty parameters $\mu_{u}=100$ ensure that the amount of supplied and discharged

\footnotetext{
${ }^{5}$ https://youtu.be/4c85DeaAhsA
} 


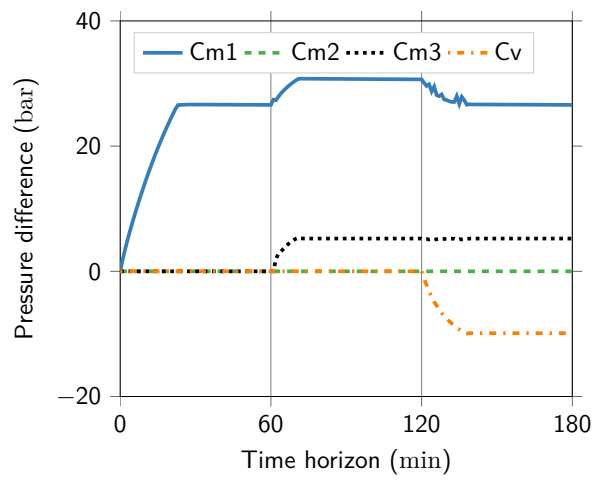

Figure 5.7: Pressure difference profile over the time horizon at active elements.

mass flows stay almost constant over time. In Figure 5.6a we see that the desired state is achieved after less than half an hour.

The main burden lies on the compressor machine $\mathrm{Cm} 1$ that directly starts compression; see Figure 5.7. To this end, it uses the gas in its inflow edges to obtain the required downstream pressure, which yields a pressure drop at the upstream entry nodes as it can be seen in Figure 5.6a. In Figure 5.6b, it is also visible that all nodes shortly deliver more or withdraw less gas to increase the overall amount of gas in the network and, thus, the pressure level. Taking a last look at Figure 5.6a, we also see that all downstream pressures of $\mathrm{Cm} 1$ stabilize in a similar way as the outflow pressure at T1 yielding a new stationary state.

At the beginning of the second hour, we reset $\nu_{\mathrm{T} 1}=1$ and set a lower bound $p_{\mathrm{T} 1}^{-}=65$ to prevent the algorithm from controlling back to the initial state. Additionally, we set $\hat{p}_{\mathrm{T} 2}=70$ and $\nu_{\mathrm{T} 2}=1000$ to reach a higher pressure for the exit customer at T2. On the way to achieve this goal, similar phenomena as before can be observed. The compressor machine $\mathrm{Cm} 3$ also starts to compress; see Figure 5.6a and Figure 5.7. As before, this leads to decreased pressures in the upstream pipes of $\mathrm{Cm} 3$, which is the reason why the already running compressor machine $\mathrm{Cm} 1$ further increases its compression ratio in order to preserve the 65 bar at $\mathrm{T} 1$ of the previous control period. Activating $\mathrm{Cm} 3$ instead of $\mathrm{Cm} 2$ follows the intuition that the shortest control period required is achieved if the "nearest" active network device is used. The in- and outflows are also used shortly to fill the pipes with additional gas to boost the pressure level throughout the network before all in- and outflow pressure and mass flow profiles stabilize in a new stationary state. 


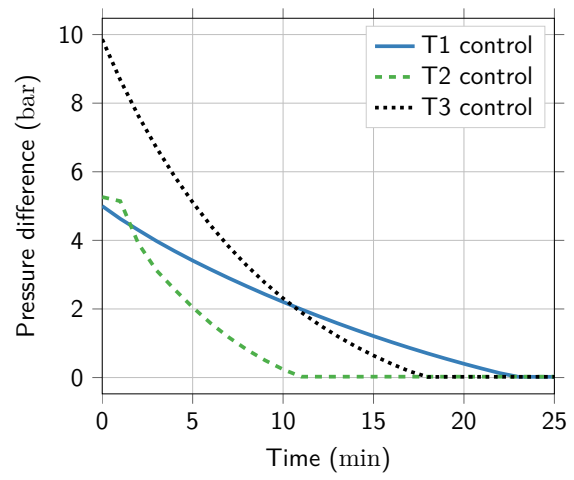

Figure 5.8: Pressure difference profile (initial vs. target) over time for three time blocks.

At the beginning of the third hour, we reset $\nu_{\mathrm{T} 2}=1$ and set a lower bound $p_{\mathrm{T} 2}^{-}=70$ to prevent the algorithm from controlling back to the initial state. Moreover, we set $\hat{p}_{\mathrm{T} 3}=55$ and $\nu_{\mathrm{T} 3}=1000$ to reach a lower pressure for the exit customer at T3. The main device used to reach this state is the control valve $\mathrm{Cv}$. It directly starts to decrease its outflow pressure to the required level; see Figure 5.6a and Figure 5.7. This would yield to increased pressures in the upstream pipes of $\mathrm{Cv}$, which, in turn, is directly balanced by the upstream compressor machine $\mathrm{Cm} 1$; see Figure 5.7. This compressor machine does not need to preserve its high pressure ratio of the previous control period. As a consequence, the pressure values at the upstream entry nodes of $\mathrm{Cm} 1$ increase; see Figure 5.6a.

At the beginning of the of third control period we observe some irregular behavior of the compressor machine $\mathrm{Cm} 1$. This chattering is not a desirable behavior, especially for active compressors, but nothing in our model avoids this behavior, especially because the max-norm objective function typically does not yield unique solutions in an MIP context. However, regularizing objective terms and/or constraints that smooth out these aspects can easily be added to our model if required.

To conclude our discussion on the ability of our instantaneous control approach, we plot the pressure differences of the respective initial and desired target state over time in Figure 5.8. It can clearly be seen that, despite the behavior in control period 2 at the beginning of that period, we always observe a slightly superlinear decrease in the difference to the target state.

All 180 MIPs of the separate time steps are of same size: 2316 variables and 2460 constraints. The constraint matrix has 7735 non-zeros. Gurobi's presolve removes approximately 280 variables and 240 constraints. 


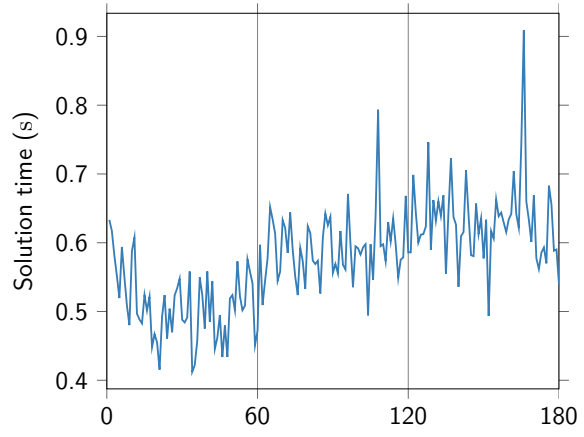

Figure 5.9: Gurobi's running times for solving all time-discrete MIPs $\left(x\right.$-axis: $\left.t_{1}, \ldots, t_{180}\right)$ of the instantaneous control method.

Approximately $5 \%$ of all variables are binaries. We remark that a fully coupled time-discretized problem without the time decomposition would consist of more than 400000 constraints with additional nonlinearities and almost 450000 variables, out of which approximately 20000 would be binary, and additional nonlinearities. The comparably small size of the single MIPs for every time step yield fast solution times. The running times of all 180 MIPs are given in Figure 5.9. All of them lie between $0.4 \mathrm{~s}$ to $1.0 \mathrm{~s}$ with an aggregated running time of $104 \mathrm{~s}$. 



\section{Summary and Outlook}

The relevant concepts of mathematical programming are cited in Chapter 2 . They build the foundation for the new developments in Chapter 4 and Chapter 5. Furthermore, Chapter 3 explains the gas transport optimization model used for the numerical results. We now emphasize the scientific achievements referring to the challenges of Section 1.2.

The first challenge is addressed in Chapter 4. In this case, we ask for algorithms to solve general problems (1.1) where the constraint functions in (1.1c) are not given analytically. In Sections 4.1-4.6, we present new tailored Algorithms 4.1-4.3 dependent on different assumptions about the underlying constraint functions. Additionally, we prove their correct and finite terminations in Theorem 4.4.7, Theorem 4.5.5, and Theorem 4.6.4, respectively. The algorithms decompose the problem into a master problem and a subproblem and rely on mixed-integer linear programming. We relate our algorithms to the existing literature in Section 4.7 and discuss the presented algorithms in Section 4.8. The theoretical study is enriched by promising numerical results for stationary gas transport optimization problems in Section 4.9.

The second challenge is addressed in Chapter 5, where we solve transient gas transport optimization problems. In Section 5.1, we introduce an instantaneous control algorithm that decomposes the problem into single time steps. Moreover, we refer to the existing literature in Section 5.2 and discuss the presented algorithm in Section 5.3. In Section 5.4, we present promising numerical results for transient gas transport optimization problems. For this purpose, a new mixed implicit-explicit Euler discretization that ensures mixed-integer linear programs is developed.

Taken all together, we give new and innovative answers to the challenges formulated in Section 1.2. We apply the methods to gas transport optimization problems taking into account our guidelines of decomposing the problems and using mixed-integer programs.

Despite these result, there are still open questions to answer. The algorithms in Chapter 4 are tailored for one-dimensional functions that we obtain after factorization. According to Remark 4.8.8, a direct transfer to 
higher dimensional functions is not possible and one focus of our future work is to develop an enhancement that removes this limitation; see Remark 4.8.9 and Schmidt et al. [142]. Moreover, the assumptions in Section 4.2 cry out for extensions. For example, we consider (in)exact local Lipschitz constants in our article Schmidt et al. [141]; see Remark 4.8.10. Additionally, the case of inexact function evaluations without a-priorily known error bounds will be investigated, as such evaluations frequently arise for mixed-integer optimal control problems with PDEs.

In Chapter 5, computationally studies of the implicit Euler discretization and the mixed implicit-explicit Euler discretization of the source terms will be realized. Note that the former yields a mixed-integer nonlinear program; see Figure 5.2. Additionally, a possible extension is to consider larger time windows to obtain better solutions; see Remark 5.3.2. In this case, the question arises whether there are suitable, i.e., theoretically rigorous and computationally tractable, generalizations of our method. 


\section{Bibliography}

[1] AG Energiebilanzen e.V. Arbeitsgemeinschaft Energiebilanzen e.V. URL: http://www.ag-energiebilanzen.de/ (visited on 04/27/2018).

[2] N. Altmüller, L. Grüne, and K. Worthmann. "Instantaneous control of the linear wave equation." In: Proceedings of MTNS. 2010. URL: http: //num.math.uni-bayreuth.de/de/publications/2010/altmueller gruene_worthmann_mtns_2010/altmueller_gruene_worthmann_ mtns_2010.pdf (visited on 04/27/2018).

[3] H. Antil, M. Hintermüller, R. Nochetto, T. Surowiec, and D. Wegner. "Finite horizon model predictive control of electrowetting on dielectric with pinning." In: Interfaces Free Bound. 19.1 (2017), pp. 1-30. DOI: 10.4171/IFB/375.

[4] S. Arora and B. Barak. Computational Complexity - A Modern Approach. New York, NY, USA: Cambridge University Press, 2009.

[5] D. Asendorpf. "Verschmähter Weltmeister." In: Die ZEIT (Apr. 2014). URL: http://www.zeit.de/2014/15/gaskraftwerk-energiewendeumwelt (visited on 04/27/2018).

[6] M. Baes, A. Del Pia, Y. Nesterov, S. Onn, and R. Weismantel. "Minimizing Lipschitz-continuous strongly convex functions over integer points in polytopes." In: Mathematical Programming 134.1 (2012), pp. 305-322. DOI: 10.1007/s10107-012-0545-8.

[7] M. K. Banda and M. Herty. "Multiscale modeling for gas flow in pipe networks." In: Mathematical Methods in the Applied Sciences 31 (Aug. 2008), pp. 915-936. DOI: 10.1002/mma.948.

[8] M. K. Banda, M. Herty, and A. Klar. "Gas flow in pipeline networks." In: Networks and Heterogeneous Media 1.1 (Mar. 2006), pp. 41-56. DOI: $10.3934 / \mathrm{nhm} .2006 .1 .41$.

[9] Baron. Branch and Reduce Optimization Navigator. URL: http:// archimedes.cheme.cmu.edu/?q=baron (visited on 04/27/2018). 
[10] B. Baumrucker and L. Biegler. "MPEC strategies for cost optimization of pipeline operations." In: Computers \& Chemical Engineering 34.6 (2010), pp. 900-913. DOI: 10.1016/j.compchemeng.2009.07.012.

[11] P. Belotti, J. Lee, L. Liberti, F. Margot, and A. Wächter. "Branching and Bounds Tightening techniques for Non-convex MINLP." In: Optimization Methods Software 24.4-5 (Aug. 2009), pp. 597-634. DOI: 10.1080/10556780903087124.

[12] P. Belotti. "Disjunctive Cuts for Nonconvex MINLP." In: Mixed Integer Nonlinear Programming. Ed. by J. Lee and S. Leyffer. New York, NY: Springer New York, 2012, pp. 117-144. DOI: 10.1007/9781-4614-1927-3_5.

[13] P. Belotti, C. Kirches, S. Leyffer, J. Linderoth, J. Luedtke, and A. Mahajan. "Mixed-integer nonlinear optimization." In: Acta Numerica 22 (2013), pp. 1-131. DOI: 10.1017/S0962492913000032.

[14] J. F. Benders. "Partitioning Procedures for Solving Mixed-variables Programming Problems." In: Numerische Mathematik 4.1 (Dec. 1962), pp. 238-252. DOI: 10.1007/BF01386316.

[15] R. E. Bixby. "Solving Real-World Linear Programs: A Decade and More of Progress." In: Operations Research 50.1 (2002), pp. 3-15. DOI: 10.1287 /opre.50.1.3.17780.

[16] "State of the Climate in 2015." In: Bulleting of the American Meteorological Society 97.8 (2015). Ed. by J. Blunden and D. Arndt. DoI: 10.1175/2016BAMSStateoftheClimate.1.

[17] P. Bonami, G. Cornuéjols, A. Lodi, and F. Margot. "A Feasibility Pump for mixed integer nonlinear programs." In: Mathematical Programming 119.2 (2009), pp. 331-352. DOI: 10.1007/s10107-008-02122.

[18] Bonmin. Basic Open-source Nonlinear Mixed INteger programming. URL: https://projects.coin-or.org/Bonmin (visited on 04/27/2018).

[19] J. Brouwer, I. Gasser, and M. Herty. "Gas Pipeline Models Revisited: Model Hierarchies, Nonisothermal Models, and Simulations of Networks." In: Multiscale Modeling \& Simulation 9.2 (2011), pp. 601-623. DOI: $10.1137 / 100813580$.

[20] C. Buchheim, C. Meyer, and R. Schäfer. Combinatorial Optimal Control of Semilinear Elliptic PDEs. Tech. rep. Nov. 2017. URL: http: //www.optimization-online.org/DB_HTML/2015/10/5161.html (visited on 04/27/2018). 
[21] Bundesgesetzblatt. "Dreizehntes Gesetz zur Änderung des Atomgesetzes." In: Bundesgesetzblatt 43 (2011), pp. 1704-1705. URL: http: //www.bgbl.de/xaver/bgbl/start.xav?startbk=Bundesanzeiger BGBl\&jumpTo=bgbl111s1704.pdf (visited on 04/27/2018).

[22] Bundesgesetzblatt. "Gesetz für den Vorrang Erneuerbarer Energien (Erneuerbare-Energien-Gesetz - EEG) sowie zur Änderung des Energiewirtschaftsgesetzes und des Mineralölsteuergesetzes." In: Bundesgesetzblatt 13 (2000), pp. 305-309. URL: http://www.bgbl.de/ xaver $/$ bgbl/start.xav?startbk $=$ Bundesanzeiger_BGBl\&jumpTo= bgbl100s0305.pdf (visited on 04/27/2018).

[23] S. Burer and A. N. Letchford. "Non-convex mixed-integer nonlinear programming: A survey." In: Surveys in Operations Research and Management Science 17.2 (2012), pp. 97-106. DOI: 10.1016/j.sorms. 2012.08.001.

[24] R. Burlacu, B. Geißler, and L. Schewe. Solving Mixed-Integer Nonlinear Programs using Adaptively Refined Mixed-Integer Linear Programs. Tech. rep. June 2017. URL: http://www.optimization-online. org/DB_HTML/2017/05/6029.html (visited on 04/27/2018).

[25] J. Carlson, A. Jaffe, and A. Wiles, eds. The Millenium Prize Problems. Providence, RI, USA: American Mathematical Society and Clay Mathematics Institute, 2006. URL: http:// www.claymath.org/ library/monographs/MPPc.pdf (visited on 04/27/2018).

[26] R. G. Carter. "Pipeline optimization: Dynamic programming after 30 years." In: Proceedings of the 30th PSIG Annual Meeting (Nov. 1998). URL: https://www.onepetro.org/conference-paper/PSIG-9803.

[27] B. Chachuat, A. B. Singer, and P. I. Barton. "Global mixed-integer dynamic optimization." In: AIChE Journal 51.8 (2005), pp. 22352253. DOI: 10.1002 /aic. 10494 .

[28] H. Choi, M. Hinze, and K. Kunisch. "Instantaneous control of backward-facing step flows." In: Applied Numerical Mathematics 31.2 (1999), pp. 133-158. DOI: 10.1016/S0168-9274(98)00131-7.

[29] H. Choi, R. Temam, P. Moin, and J. Kim. "Feedback control for unsteady flow and its application to the stochastic Burgers equation." In: Journal of Fluid Mechanics 253 (1993), pp. 509-543. DOI: 10. 1017/S0022112093001880.

[30] V. Chvátal. Linear Programming (Series of Books in the Mathematical Sciences). New York, New York: W. H. Freeman, 1983. 
[31] E. A. Coddington and R. Carlson. Linear ordinary differential equations. SIAM, 1997. URL: http://gibbs.if.usp.br/ marchett/fismat2/ linear-ode_coddington-carlson.pdf (visited on 04/27/2018).

[32] C. F. Colebrook. "Turbulent flow in pipes with particular reference to the transition region between smooth and rough pipe laws." In: Journal of the Institution of Civil Engineers 11 (Feb. 1939), pp. 133156. DOI: $10.1680 /$ ijoti.1939.13150.

[33] M. Conforti, G. Cornuejols, and G. Zambelli. Integer Programming. Vol. 1. Springer, 2014. URL: https://www.springer.com/us/book/ 9783319110073 (visited on 04/27/2018).

[34] S. A. Cook. "The Complexity of Theorem-proving Procedures." In: Proceedings of the Third Annual ACM Symposium on Theory of Computing. STOC '71. Shaker Heights, Ohio, USA: ACM, 1971, pp. 151-158. DOI: $10.1145 / 800157.805047$.

[35] Couenne. Convex Over and Under ENvelopes for Nonlinear Estimation. URL: https:// projects.coin-or.org/Couenne (visited on 04/27/2018).

[36] Cplex. IBM ILOG CPLEX Optimization Studio. URL: https://www01.ibm.com/software/commerce / optimization/cplex-optimizer/ (visited on $04 / 27 / 2018$ ).

[37] H. Crowder and M. W. Padberg. "Solving Large-Scale Symmetric Travelling Salesman Problems to Optimality." In: Management Science 26.5 (1980), pp. 495-509. DOI: 10.1287/mnsc.26.5.495.

[38] R. J. Dakin. "A tree-search algorithm for mixed integer programming problems." In: The Computer Journal 8.3 (1965), pp. 250-255. DOI: 10.1093/comjnl/8.3.250.

[39] G. B. Dantzig. "A History of Scientific Computing." In: ed. by S. G. Nash. New York, NY, USA: ACM, 1990. Chap. Origins of the Simplex Method, pp. 141-151. URL: http://doi.acm.org/10.1145/87252.88081 (visited on 04/27/2018).

[40] G. B. Dantzig. "Linear Programming." In: Proceedings of the Symposium on Modern Calculating Machinery and Numerical Methods, UCLA (July 29-31, 1948). Appl. Math. 15. National Bureau of Standards, 1951, pp. 18-21. 
[41] G. B. Dantzig. "On the Significance of Solving Linear Programming Problems with Some Integer Variables." In: Econometrica 28.1 (1960), pp. 30-44. URL: http://www.jstor.org/stable/1905292 (visited on 04/27/2018).

[42] G. B. Dantzig and M. N. Thapa. Linear Programming 1: Introduction. New York, New York: Springer, 1997. URL: http://www.springer. com/de/book/9780387948331 (visited on 04/27/2018).

[43] G. B. Dantzig and M. N. Thapa. Linear Programming 2: Theory and Extensions. New York, New York: Springer, 1997. URL: http://www. springer.com/de/book/9780387986135 (visited on 04/27/2018).

[44] G. B. Dantzig and P. Wolfe. "Decomposition Principle for Linear Programs." In: Operations Research 8.1 (Feb. 1960), pp. 101-111. DOI: $10.1287 /$ opre.8.1.101.

[45] S. Dempe. Foundations of bilevel programming. Springer Science \& Business Media, 2002. DOI: 10.1007/b101970.

[46] S. Dempe, V. Kalashnikov, G. A. Pérez-Valdés, and N. Kalashnykova. Bilevel Programming Problems. Theory, Algorithms and Applications to Energy Networks. Springer-Verlag Berlin Heidelberg, 2015. DOI: 10.1007/978-3-662-45827-3.

[47] DESFA. National Natural Gas System Operator S.A. URL: http: //www.desfa.gr (visited on 04/27/2018).

[48] P. Domschke, B. Geißler, O. Kolb, J. Lang, A. Martin, and A. Morsi. "Combination of Nonlinear and Linear Optimization of Transient Gas Networks." In: INFORMS Journal on Computing 23.4 (2011), pp. 605-617. DOI: 10.1287/ijoc.1100.0429.

[49] P. Domschke, B. Hiller, J. Lang, and C. Tischendorf. Modellierung von Gasnetzwerken: Eine Übersicht. Tech. rep. Oct. 2017. URL: https: //opus4.kobv.de/opus4-trr154/frontdoor/index/index/docId/191 (visited on $04 / 27 / 2018$ ).

[50] M. A. Duran and I. E. Grossmann. "An outer-approximation algorithm for a class of mixed-integer nonlinear programs." In: Mathematical Programming 36.3 (1986), pp. 307-339. DOI: 10.1007/BF02592064.

[51] K. Ehrhardt and M. C. Steinbach. "KKT Systems in Operative Planning for Gas Distribution Networks." In: Proc. Appl. Math. Mech. 4.1 (Dec. 2004), pp. 606-607. DOI: 10.1002/pamm.200410284. 
[52] K. Ehrhardt and M. C. Steinbach. "Nonlinear Optimization in Gas Networks." In: Modeling, Simulation and Optimization of Complex Processes. Ed. by H. G. Bock, E. Kostina, H. X. Phu, and R. Rannacher. Springer: Berlin, 2005, pp. 139-148. DOI: 10.1007/3-54027170-8_11.

[53] Y. G. Evtuschenko. "Numerical methods for finding global extrema." In: USSR Computational Mathematics and Mathematical Physics 11 (1971), pp. 38-54. DOI: 10.1016/0041-5553(71)90065-6.

[54] M. M. Feistauer. Mathematical methods in fluid dynamics. Pitman monographs and surveys in pure and applied mathematics. Harlow, Essex, England: Longman Scientific \& Technical New York, 1993.

[55] R. Fletcher and S. Leyffer. "Solving mixed integer nonlinear programs by outer approximation." In: Mathematical Programming 66.1 (1994), pp. 327-349. DOI: 10.1007/BF01581153.

[56] L. Fortnow. "The Status of the P Versus NP Problem." In: Commun. ACM 52.9 (Sept. 2009), pp. 78-86. DOI: 10.1145/1562164.1562186.

[57] A. Fügenschuh, B. Geißler, R. Gollmer, A. Morsi, M. E. Pfetsch, J. Rövekamp, M. Schmidt, K. Spreckelsen, and M. C. Steinbach. "Physical and technical fundamentals of gas networks." In: ed. by M. E. Pfetsch, T. Koch, L. Schewe, and B. Hiller. Philadelphia, PA, USA: Society for Industrial and Applied Mathematics, 2015. Chap. 2, pp. 17-44. DOI: 10.1137/1.9781611973693.ch2.

[58] A. Fügenschuh, B. Geißler, A. Martin, and A. Morsi. "The Transport PDE and Mixed-Integer Linear Programming." In: Models and Algorithms for Optimization in Logistics. Ed. by C. Barnhart, U. Clausen, U. Lauther, and R. H. Möhring. Dagstuhl Seminar Proceedings 09261. Dagstuhl, Germany: Schloss Dagstuhl - Leibniz-Zentrum fuer Informatik, Germany, 2009. URL: http://drops.dagstuhl.de/ opus/volltexte/2009/2167 (visited on 04/27/2018).

[59] M. R. Garey and D. S. Johnson. Computers and Intractability: A Guide to the Theory of NP-Completeness. New York, NY, USA: W. H. Freeman \& Co., 1979.

[60] B. Geißler. "Towards Globally Optimal Solutions for MINLPs by Discretization Techniques with Applications in Gas Network Optimization." PhD thesis. Friedrich-Alexander-Universität ErlangenNürnberg, 2011. URL: http://www.dr.hut-verlag.de/9783843901680. html (visited on 04/27/2018). 
[61] B. Geißler, O. Kolb, J. Lang, G. Leugering, A. Martin, and A. Morsi. "Mixed integer linear models for the optimization of dynamical transport networks." In: Mathematical Methods of Operations Research 73.3 (2011), pp. 339-362. DOI: 10.1007/s00186-011-0354-5.

[62] B. Geißler, A. Martin, A. Morsi, and L. Schewe. "The MILPrelaxation approach." In: ed. by M. E. Pfetsch, T. Koch, L. Schewe, and B. Hiller. Philadelphia, PA, USA: Society for Industrial and Applied Mathematics, 2015. Chap. 6, pp. 103-122. DOI: 10.1137/1. 9781611973693.ch6.

[63] B. Geißler, A. Martin, A. Morsi, and L. Schewe. "Using Piecewise Linear Functions for Solving MINLPs." In: Mixed Integer Nonlinear Programming. Ed. by J. Lee and S. Leyffer. The IMA Volumes in Mathematics and its Applications. New York, NY: Springer New York, 2012, pp. 287-314. DOI: 10.1007/978-1-4614-1927-3_10.

[64] B. Geißler, A. Morsi, and L. Schewe. "A New Algorithm for MINLP Applied to Gas Transport Energy Cost Minimization." In: Facets of Combinatorial Optimization: Festschrift for Martin Grötschel. Ed. by M. Jünger and G. Reinelt. Berlin, Heidelberg: Springer Berlin Heidelberg, 2013, pp. 321-353. DOI: 10.1007/978-3-642-38189-8_14.

[65] B. Geißler, A. Morsi, L. Schewe, and M. Schmidt. "Penalty Alternating Direction Methods for Mixed-Integer Optimization: A New View on Feasibility Pumps." In: SIAM Journal on Optimization 27.3 (2017), pp. 1611-1636. DOI: 10.1137/16M1069687.

[66] B. Geißler, A. Morsi, L. Schewe, and M. Schmidt. "Solving Highly Detailed Gas Transport MINLPs: Block Separability and Penalty Alternating Direction Methods." In: INFORMS Journal on Computing (2017). URL: http://www.optimization-online.org/DB_HTML/2016/ 06/5523.html (visited on 04/27/2018). Forthcoming.

[67] B. Geißler, A. Morsi, L. Schewe, and M. Schmidt. "Solving powerconstrained gas transportation problems using an MIP-based alternating direction method." In: Computers 83 Chemical Engineering 82 (2015), pp. 303-317. DOI: 10.1016/j.compchemeng.2015.07.005.

[68] A. M. Geoffrion. "Generalized Benders decomposition." In: Journal of Optimization Theory and Applications 10.4 (1972), pp. 237-260. DOI: $10.1007 / \mathrm{BF} 00934810$. 
[69] R. E. Gomory. "An algorithm for integer solutions to linear programming." In: Recent Advances in Mathematical Programming (1969). Ed. by R. Graves and P. Wolfe, pp. 269-302. URL: http://www. ralphgomory.com/wp-content/uploads/2011/10/18-An-Algorithmfor-Integer-Solutions- to- Linear- Programs- Recent- Advances-inMathematical-Programming.pdf (visited on 04/27/2018).

[70] R. E. Gomory. An algorithm for the mixed-integer problem. Tech. rep. The RAND Cooperation, 1960. URL: http://www.dtic.mil/get-trdoc/pdf?AD=AD0616505 (visited on 04/27/2018).

[71] R. E. Gomory. "Solving linear programming problems in integers." In: Combinatorial analysis, Proceedings 10 (1960). Ed. by R. Bellman and M. Hall. URL: http://pages.stern.nyu.edu/ rgomory/academic_ papers/08_LP.pdf (visited on 04/27/2018).

[72] U. Gotzes, N. Heinecke, B. Hiller, J. Rövekamp, and T. Koch. "Regulatory rules for gas markets in Germany and other European countries." In: ed. by M. E. Pfetsch, T. Koch, L. Schewe, and B. Hiller. Philadelphia, PA, USA: Society for Industrial and Applied Mathematics, 2015. Chap. 3, pp. 45-64. DOI: 10.1137/1.9781611973693.ch3.

[73] M. Gugat, M. Herty, and V. Schleper. "Flow control in gas networks: Exact controllability to a given demand." In: Mathematical Methods in the Applied Sciences 34.7 (2011), pp. 745-757. DOI: 10.1002/mma. 1394.

[74] M. Gugat. "Boundary Controllability between Sub- and Supercritical Flow." In: SIAM Journal on Control and Optimization 42.3 (2003), pp. 1056-1070. DOI: 10.1137/S0363012902409660.

[75] M. Gugat, F. M. Hante, M. Hirsch-Dick, and G. Leugering. "Stationary states in gas networks." In: Networks and Heterogeneous Media 10.2 (2015), pp. 295-320. DOI: 10.3934/nhm.2015.10.295.

[76] M. Gugat, G. Leugering, A. Martin, M. Schmidt, M. Sirvent, and D. Wintergerst. "MIP-Based Instantaneous Control of Mixed-Integer PDE-Constrained Gas Transport Problems." In: Computational Optimization and Applications 70.1 (May 2018), pp. 267-294. DOI: 10.1007/s10589-017-9970-1.

[77] M. Gugat, G. Leugering, A. Martin, M. Schmidt, M. Sirvent, and D. Wintergerst. "Towards Simulation Based Mixed-Integer Optimization with Differential Equations." In: Networks (2018). DOI: 10.1002/net. 21812. 
[78] M. Gugat, R. Schultz, and D. Wintergerst. "Networks of pipelines for gas with nonconstant compressibility factor: stationary states." In: Computational and Applied Mathematics (2016), pp. 1-32. DOI: 10.1007/s40314-016-0383-z.

[79] O. K. Gupta and A. Ravindran. "Branch and Bound Experiments in Convex Nonlinear Integer Programming." In: Management Science 31.12 (1985), pp. 1533-1546. DOI: 10.1287/mnsc.31.12.1533.

[80] Gurobi. Gurobi Optimization Inc. URL: http://www.gurobi.com (visited on $04 / 27 / 2018$ ).

[81] M. Hahn, S. Leyffer, and V. M. Zavala. Mixed-Integer PDEConstrained Optimal Control of Gas Networks. Tech. rep. Aug. 2017. URL: https://wiki.mcs.anl.gov/leyffer/images/2/27/GasNetMIP.pdf (visited on 04/27/2018).

[82] P. Hansen and B. Jaumard. "Lipschitz optimization." In: Handbook of Global Optimization. Ed. by R. Horst and P. M. Pardalos. Springer US, 1995, pp. 407-493. DOI: 10.1007/978-1-4615-2025-2_9.

[83] F. M. Hante, G. Leugering, A. Martin, L. Schewe, and M. Schmidt. "Challenges in Optimal Control Problems for Gas and Fluid Flow in Networks of Pipes and Canals: From Modeling to Industrial Applications." In: Industrial Mathematics and Complex Systems: Emerging Mathematical Models, Methods and Algorithms. Ed. by P. Manchanda, R. Lozi, and A. H. Siddiqi. Singapore: Springer Singapore, 2017, pp. 77-122. DOI: 10.1007/978-981-10-3758-0_5.

[84] M. Herty, C. Kirchner, and A. Klar. "Instantaneous control for traffic flow." In: Mathematical Methods in the Applied Sciences 30.2 (2007), pp. 153-169. DOI: 10.1002/mma.779.

[85] M. Hinze. "Optimal and instantaneous control of the instationary Navier-Stokes equations." Technische Universität Dresden, 2002. URL: https://www.math.uni-hamburg.de/home/hinze/Psfiles/habil_mod. pdf (visited on $04 / 27 / 2018$ ).

[86] R. Horst. "Deterministic global optimization with partition sets whose feasibility is not known: Application to concave minimization, reverse convex constraints, DC-programming, and Lipschitzian optimization." In: Journal of Optimization Theory and Applications 58.1 (1988), pp. 11-37. DOI: 10.1007/BF00939768. 
[87] R. Horst and N. V. Thoai. "Branch-and-bound methods for solving systems of Lipschitzian equations and inequalities." In: Journal of Optimization Theory and Applications 58.1 (1988), pp. 139-145. DOI: 10.1007/BF00939776.

[88] R. Horst and H. Tuy. Global Optimization. 2nd. Berlin: Springer Verlag, 1996. URL: https://www.springer.com/la/book/9783540610380.

[89] R. Horst and H. Tuy. "On the convergence of global methods in multiextremal optimization." In: Journal of Optimization Theory and Applications 54.2 (1987), pp. 253-271. DOI: 10.1007/BF00939434.

[90] R. Hundhammer and G. Leugering. "Instantaneous Control of Vibrating String Networks." In: Online Optimization of Large Scale Systems. Ed. by M. Grötschel, S. O. Krumke, and J. Rambau. Berlin, Heidelberg: Springer Berlin Heidelberg, 2001, pp. 229-249. DOI: 10.1007/978-3-662-04331-8_15.

[91] S. Jacobsson and V. Lauber. "The politics and policy of energy system transformation - explaining the German diffusion of renewable energy technology." In: Energy Policy 34.3 (2006). Renewable Energy Policies in the European UnionRenewable Energy Policies in the European Union, pp. 256-276. DOI: 10.1016/j.enpol.2004.08.029.

[92] R. G. Jeroslow. "There Cannot be any Algorithm for Integer Programming with Quadratic Constraints." In: Operations Research 21.1 (1973), pp. 221-224. URL: http://www.jstor.org/stable/169101 (visited on $04 / 27 / 2018$ ).

[93] I. Joormann, M. Schmidt, M. C. Steinbach, and B. M. Willert. "What does "feasible" mean?" In: ed. by M. E. Pfetsch, T. Koch, L. Schewe, and B. Hiller. Philadelphia, PA, USA: Society for Industrial and Applied Mathematics, 2015. Chap. 11, pp. 211-232. DOI: 10.1137/1. 9781611973693.ch11.

[94] N. B. Karmarkar. "A new polynomial-time algorithm for linear programming." In: Combinatorica 4.4 (1984), pp. 373-395. DOI: 10. 1007/BF02579150.

[95] R. M. Karp. "Reducibility among Combinatorial Problems." In: Complexity of Computer Computations: Proceedings of a symposium on the Complexity of Computer Computations, held March 20-22, 1972, at the IBM Thomas J. Watson Research Center, Yorktown Heights, New York, and sponsored by the Office of Naval Research, Mathematics Program, IBM World Trade Corporation, and the IBM 
Research Mathematical Sciences Department. Ed. by R. E. Miller, J. W. Thatcher, and J. D. Bohlinger. Boston, MA: Springer US, 1972, pp. 85-103. DOI: 10.1007/978-1-4684-2001-2_9.

[96] L. G. Khachiyan. "A polynomial algorithm in linear programming." In: Doklady Akademiia Nauk SSSR 244 (1979), pp. 1093-1096. URL: http://ci.nii.ac.jp/naid/10011277264/en/ (visited on 04/27/2018).

[97] L. G. Khachiyan. "Polynomial algorithms in linear programming." In: USSR Computational Mathematics and Mathematical Physics 20.1 (1980), pp. 53-72. DOI: 10.1016/0041-5553(80)90061-0.

[98] V. Klee and G. J. Minty. "How good is the simplex algorithm?" In: Inequalities. Ed. by O. Shisha. Vol. III. New York: Academic Press, 1972, pp. 159-175. URL: http:// www.scirp.org / (S(i43dyn45teexjx455qlt3d2q))/reference/ReferencesPapers.aspx? ReferenceID=610763 (visited on 04/27/2018).

[99] T. Koch, B. Hiller, M. Pfetsch, and L. Schewe. Evaluating Gas Network Capacities. Ed. by M. E. Pfetsch, T. Koch, L. Schewe, and B. Hiller. Philadelphia, PA, USA: Society for Industrial and Applied Mathematics, 2015. DOI: 10.1137/1.9781611973693.

[100] G. R. Kocis and I. E. Grossmann. "Relaxation strategy for the structural optimization of process flow sheets." In: Industrial \& Engineering Chemistry Research 26.9 (1987), pp. 1869-1880. DOI: 10.1021/ie00069a026.

[101] J. Králik, P. Stiegler, Z. Vostrý, and J. Záworka. Dynamic Modeling of Large-Scale Networks with Application to Gas Distribution. Vol. 6. Studies in Automation and Control. New York: Elsevier Sci. Publ., 1988.

[102] D. E. Kvasov and Y. D. Sergeyev. "Lipschitz global optimization methods in control problems." In: Automation and Remote Control 74.9 (2013), pp. 1435-1448. DOI: 10.1134/S0005117913090014.

[103] LaMaTTO++. LaMaTTO++: A Framework for Modeling and Solving Mixed-Integer Nonlinear Programming Problems on Networks. URL: http://www.mso.math.fau.de/edom/projects/lamatto.html (visited on 04/27/2018).

[104] A. H. Land and A. G. Doig. "An Automatic Method of Solving Discrete Programming Problems." In: Econometrica 28.3 (1960), pp. 497-520. DOI: 10.2307/1910129. 
[105] J. Lelieveld, D. Kunkel, and M. G. Lawrence. "Global risk of radioactive fallout after major nuclear reactor accidents." In: Atmospheric Chemistry and Physics 12.9 (2012), pp. 4245-4258. DOI: 10.5194/acp12-4245-2012.

[106] G. Leugering, A. Martin, M. Schmidt, and M. Sirvent. "Nonoverlapping Domain Decomposition for Optimal Control Problems governed by Semilinear Models for Gas Flow in Networks." In: Control $\&$ Cybernetics 46.3 (2017), pp. 191-225.

[107] S. Leyffer. "Deterministic Methods for Mixed Integer Nonlinear Programming." PhD thesis. University of Dundee, Dundee, Scotland, UK, 1993. URL: https: / / pdfs . semanticscholar .org / 3d19 / d40fb5d43c81085e173f96053c925009372d.pdf (visited on 04/27/2018).

[108] L. Liberti and C. C. Pantelides. "Convex Envelopes of Monomials of Odd Degree." In: Journal of Global Optimization 25.2 (2003), pp. 157-168. DOI: 10.1023/A:1021924706467.

[109] M. V. Lurie. Modeling of Oil Product and Gas Pipeline Transportation. Wiley-VCH, 2008. URL: http://eu.wiley.com/WileyCDA/WileyTitle/ productCd-3527408339.html (visited on 04/27/2018).

[110] S. J. Maher, T. Fischer, T. Gally, G. Gamrath, A. Gleixner, R. L. Gottwald, G. Hendel, T. Koch, M. E. Lübbecke, M. Miltenberger, B. Müller, M. E. Pfetsch, C. Puchert, D. Rehfeldt, S. Schenker, R. Schwarz, F. Serrano, Y. Shinano, D. Weninger, J. T. Witt, and J. Witzig. The SCIP Optimization Suite 4.0. Tech. rep. 17-12. Takustr. 7, 14195 Berlin: ZIB, 2017. URL: https://opus4.kobv.de/opus4zib/frontdoor/index/index/docId/6217 (visited on 04/27/2018).

[111] D. Mahlke, A. Martin, and S. Moritz. "A mixed integer approach for time-dependent gas network optimization." In: Optimization Methods and Software 25.4 (2010), pp. 625-644. DOI: 10.1080 / 10556780903270886.

[112] D. Mahlke, A. Martin, and S. Moritz. "A simulated annealing algorithm for transient optimization in gas networks." In: Mathematical Methods of Operations Research 66 (2007), pp. 99-116. DOI: 10.1007/s00186-006-0142-9.

[113] O. L. Mangasarian. Nonlinear Programming. Society for Industrial and Applied Mathematics, 1994. DOI: 10.1137/1.9781611971255. 
[114] H. M. Markowitz and A. S. Manne. "On the Solution of Discrete Programming Problems." In: Econometrica 25.1 (1957), pp. 84-110. URL: http://www.jstor.org/stable/1907744 (visited on 04/27/2018).

[115] A. Martin and M. Möller. "Cutting Planes for the Optimisation of Gas Networks." In: Modeling, Simulation and Optimization of Complex Processes. Ed. by H. G. Bock, E. Kostina, H. X. Phu, and R. R. Heidelberg: Springer, 2005, pp. 307-330. DOI: 10.1007/3-54027170-8_24.

[116] A. Martin, M. Möller, and S. Moritz. "Mixed Integer Models for the Stationary Case of Gas Network Optimization." In: Mathematical Programming 105.2 (2006), pp. 563-582. DOI: 10.1007/s10107-0050665-5.

[117] B. A. McCarl and T. H. Spreen. "Applied Mathematical Programming using Algebraic Systems." Unpublished Book. URL: http:// agecon2 . tamu . edu / people / faculty / mccarl- bruce / regbook. htm (visited on $04 / 27 / 2018$ ).

[118] G. P. McCormick. "Computability of global solutions to factorable nonconvex programs: Part I - Convex underestimating problems." In: Mathematical Programming 10.1 (1976), pp. 147-175. DOI: 10. 1007/BF01580665.

[119] W. McKinney. Python for Data Analysis: Data Wrangling with Pandas, NumPy, and IPython. First. Beijing: O'Reilly, 2013. URL: http:// shop. oreilly.com/ product/0636920023784.do (visited on 04/27/2018).

[120] M. Möller. "Mixed Integer Models for the Optimisation of Gas Networks in the Stationary Case." PhD thesis. Technische Universität Darmstadt, 2004. URL: http: / / citeseerx. ist.psu . edu / viewdoc / download?doi $=10.1 \cdot 1.632 .6459 \& \mathrm{rep}=$ rep1\&type $=$ pdf $($ visited on 04/27/2018).

[121] S. Moritz. "A Mixed Integer Approach for the Transient Case of Gas Network Optimization." PhD thesis. Technische Universität Darmstadt, 2006. URL: http://tuprints.ulb.tu-darmstadt.de/785/1/ diss.pdf (visited on 04/27/2018).

[122] A. Morsi. "Solving MINLPs on Loosely-Coupled Networks with Applications in Water and Gas Network Optimization." PhD thesis. Friedrich-Alexander-Universität Erlangen-Nürnberg, 2013. URL: 
http: / / www.dr.hut-verlag. de /9783843911764.html (visited on 04/27/2018).

[123] G. L. Nemhauser and L. A. Wolsey. Integer and Combinatorial Optimization. New York, NY, USA: Wiley-Interscience, 1988. URL: http://eu.wiley.com/WileyCDA/WileyTitle/productCd-0471359432. html (visited on 04/27/2018).

[124] A. J. Osiadacz. Different Transient Flow Models - Limitations, Advantages, and Disadvantages. PSIG report 9606. Pipeline Simulation Interest Group, 1996. URL: https://www.onepetro.org/conferencepaper/PSIG-9606 (visited on 04/27/2018).

[125] J. Pintér. "Branch- and bound algorithms for solving global optimization problems with Lipschitzian structure." In: Optimization 19.1 (1988), pp. 101-110. DOI: 10.1080/02331938808843322.

[126] J. Pintér. "Extended univariate algorithms for n-dimensional global optimization." In: Computing 36.1 (1986), pp. 91-103. DOI: 10.1007/ BF02238195.

[127] J. Pintér. "Global optimization on convex sets." In: OperationsResearch-Spektrum 8.4 (1986), pp. 197-202. DOI: 10.1007 / BF01721128.

[128] J. Pintér. "Globally convergent methods for n-dimensional multiextremal optimization." In: Optimization 17.2 (1986), pp. 187-202. DOI: 10.1080/02331938608843118.

[129] J. Pintér. "Nonlinear Optimization with GAMS/LGO." In: Journal of Global Optimization 38.1 (May 2007), pp. 79-101. DOI: 10.1007/ s10898-006-9084-2.

[130] J. D. Pintér. Global Optimization in Action (Continuous and Lipschitz Optimization: Algorithms, Implementations and Applications). Springer Verlag, 1996. DOI: 10.1007/978-1-4757-2502-5.

[131] S. A. Piyavskii. "An Algorithm for Finding the absolute Extremum of a Function." In: USSR Computational Mathematics and Mathematical Physics 12 (1972), pp. 57-67. DOI: 10.1016/0041-5553(72)90115-2.

[132] I. Quesada and I. E. Grossmann. "An LP NLP based branch and bound algorithm for convex MINLP optimization." In: Computers $\mathcal{E}$ Chemical Engineering 16 (1992), pp. 937-947. URL: http://repository. cmu.edu/cgi/viewcontent.cgi? article $=1176 \&$ context $=$ cheme (visited on $04 / 27 / 2018$ ). 
[133] R. Z. Ríos-Mercado and C. Borraz-Sánchez. "Optimization problems in natural gas transportation systems: A state-of-the-art review." In: Applied Energy 147 (2015), pp. 536-555. DOI: 10.1016/j.apenergy. 2015.03.017.

[134] H. S. Ryoo and N. V. Sahinidis. "A branch-and-reduce approach to global optimization." In: Journal of Global Optimization 8.2 (1996), pp. 107-138. DOI: 10.1007/BF00138689.

[135] H. S. Ryoo and N. V. Sahinidis. "Global optimization of nonconvex NLPs and MINLPs with applications in process design." In: Computers 83 Chemical Engineering 19.5 (1995), pp. 551-566. DOI: 10.1016/0098-1354(94)00097-2.

[136] S. Sager. "Numerical methods for mixed-integer optimal control problems." PhD thesis. Universität Heidelberg, 2006. URL: http://mathopt. de/PUBLICATIONS/Sager2005.pdf (visited on 04/27/2018).

[137] N. V. Sahinidis and I. E. Grossmann. "Convergence properties of generalized benders decomposition." In: Computers \& Chemical Engineering 15.7 (1991), pp. 481-491. DOI: 10.1016/0098-1354(91)85027$\mathrm{R}$.

[138] M. Schmidt. "A Generic Interior-Point Framework for Nonsmooth and Complementarity Constrained Nonlinear Optimization." PhD thesis. Leibniz Universität Hannover, 2013.

[139] M. Schmidt. "An interior-point method for nonlinear optimization problems with locatable and separable nonsmoothness." In: EURO J. Comput. Optim. 3.4 (Nov. 2015), pp. 309-348. DOI: 10.1007/s13675015-0039-6.

[140] M. Schmidt, D. Aßmann, R. Burlacu, J. Humpola, I. Joormann, N. Kanelakis, T. Koch, D. Oucherif, M. E. Pfetsch, L. Schewe, R. Schwarz, and M. Sirvent. "GasLib-A Library of Gas Network Instances." In: Data 2.4 (2017). DOI: 10.3390/data2040040.

[141] M. Schmidt, M. Sirvent, and W. Wollner. A Decomposition Method for MINLPs with Lipschitz Continuous Nonlinearities. Tech. rep. July 2017. URL: http://www.optimization-online.org/DB_HTML/2017/ 07/6130.html (visited on 04/27/2018). Submitted to Mathematical Programming.

[142] M. Schmidt, M. Sirvent, and W. Wollner. "Simulation-Based MINLPs with Lipschitz Nonlinearities." Working paper. 2017. 
[143] M. Schmidt, M. C. Steinbach, and B. M. Willert. "A Primal Heuristic for Nonsmooth Mixed Integer Nonlinear Optimization." In: Facets of Combinatorial Optimization. Ed. by M. Jünger and G. Reinelt. Berlin, Heidelberg: Springer, 2013, pp. 295-320. DOI: 10.1007/978-3642-38189-8_13.

[144] M. Schmidt, M. C. Steinbach, and B. M. Willert. "An MPEC based heuristic." In: ed. by M. E. Pfetsch, T. Koch, L. Schewe, and B. Hiller. Philadelphia, PA, USA: Society for Industrial and Applied Mathematics, 2015. Chap. 9, pp. 163-180. DOI: 10.1137/1.9781611973693.ch9.

[145] M. Schmidt, M. C. Steinbach, and B. M. Willert. "High detail stationary optimization models for gas networks." In: Optimization and Engineering 16.1 (2015), pp. 131-164. DOI: 10.1007/s11081-014-9246$\mathrm{x}$.

[146] M. Schmidt, M. C. Steinbach, and B. M. Willert. "High detail stationary optimization models for gas networks: validation and results." In: Optimization and Engineering 17.2 (2016), pp. 437-472. DOI: 10.1007/s11081-015-9300-3.

[147] A. Schrijver. Theory of Linear and Integer Programming. New York, NY, USA: John Wiley \& Sons, Inc., 1986. URL: https: / / promathmedia . files . wordpress . com / 2013 / 10 / alexander_ schrijver_theory_of_linear_and_integerbookfi-org.pdf (visited on 04/27/2018).

[148] M. Sirvent, N. Kanelakis, B. Geißler, and P. Biskas. "Linearized model for optimization of coupled electricity and natural gas systems." In: Journal of Modern Power Systems and Clean Energy 5.3 (May 2017), pp. 364-374. DOI: 10.1007/s40565-017-0275-2.

[149] E. M. Smith and C. C. Pantelides. "A symbolic reformulation/spatial branch-and-bound algorithm for the global optimisation of nonconvex MINLPs." In: Computers \& Chemical Engineering 23.4-5 (1999), pp. 457-478. DOI: 10.1016/S0098-1354(98)00286-5.

[150] E. M. Smith and C. C. Pantelides. "Global optimisation of nonconvex MINLPs." In: Computers $\& 3$ Chemical Engineering 21 (1997), S791S796. DOI: 10.1016/S0098-1354(97)87599-0.

[151] M. C. Steinbach. "On PDE Solution in Transient Optimization of Gas Networks." In: J. Comput. Appl. Math. 203.2 (June 2007), pp. 345361. DOI: 10.1016/j.cam.2006.04.018. 
[152] R. A. Stubbs and S. Mehrotra. "A branch-and-cut method for 0-1 mixed convex programming." In: Mathematical Programming 86.3 (Dec. 1999), pp. 515-532. DOI: 10.1007/s101070050103.

[153] M. Tawarmalani and N. V. Sahinidis. Convexification and Global Optimization in Continuous and Mixed-Integer Nonlinear Programming. Boston, MA: Kluwer Academic Publishers, 2002, p. 478. DOI: 10.1007/978-1-4757-3532-1.

[154] M. Tawarmalani and N. V. Sahinidis. "Global optimization of mixedinteger nonlinear programs: A theoretical and computational study." In: Mathematical Programming 99.3 (2004), pp. 563-591. DOI: 10. 1007/s10107-003-0467-6.

[155] H. Tuy and R. Horst. "Convergence and restart in branch-andbound algorithms for global optimization. Application to concave minimization and D.C. Optimization problems." In: Mathematical Programming 41.1 (1988), pp. 161-183. DOI: 10.1007/BF01580762.

[156] Umweltbundesamt. Berichterstattung unter der Klimarahmenkonvention der Vereinten Nationen und dem Kyoto-Protokoll 2017. Tech. rep. Umweltbundesamt, 2017. URL: http://www.umweltbundesamt.de/ publikationen/berichterstattung-unter-der-klimarahmenkonvention2 (visited on $04 / 27 / 2018$ ).

[157] A. Wächter and L. T. Biegler. "On the implementation of an interiorpoint filter line-search algorithm for large-scale nonlinear programming." In: Mathematical Programming 106.1 (2006), pp. 25-57. DOI: 10.1007/s10107-004-0559-y.

[158] H.-J. Wagner, M. K. Koch, J. Burkhardt, T. Große Böckmann, N. Feck, and P. Kruse. "CO2-Emissionen der Stromerzeugung - Ein ganzheitlicher Vergleich verschiedener Techniken." In: Fachzeitschrift BWK 59.8 (2007). URL: https : / / www . vdi . de / fileadmin / vdi_de / redakteur_dateien / geu_dateien / FB4 - Internetseiten / CO2-Emissionen \% 20der\% 20Stromerzeugung_01.pdf (visited on 04/27/2018).

[159] T. Westerlund and F. Pettersson. "An extended cutting plane method for solving convex MINLP problems." In: Computers \&5 Chemical Engineering 19 (1995). European Symposium on Computer Aided Process Engineering, pp. 131-136. DOI: 10.1016/0098-1354(95)87027$\mathrm{X}$. 
Bibliography

[160] L. A. Wolsey. Integer programming. New York, NY, USA: WileyInterscience, 1998. URL: http://eu.wiley.com/WileyCDA/WileyTitle/ productCd-0471283665.html (visited on 04/27/2018).

[161] A. Zlotnik, M. Chertkov, and S. Backhaus. "Optimal control of transient flow in natural gas networks." In: 2015 54th IEEE Conference on Decision and Control (CDC). Dec. 2015, pp. 4563-4570. DOI: 10.1109/CDC.2015.7402932. 
Natural gas is one of the most important energy sources. Consequently, its transportation through gas networks is an essential task and gives rise to gas transport problems. Such optimization problems involve discrete decisions to switch network elements as valves, control valves, or compressor machines. Moreover, the physical behavior of natural gas is described by differential equations. Thus, mixed-integer problems constrained by differential equations become relevant. The scientific contribution of this thesis is twofold.

First, three new global algorithms are presented. A typical solution approach transforms the differential equations to linear constraints. This is reasonable as mixed-integer linear programming is the most successful instance of mixed-integer programming. The algorithms in this thesis do not rely on this transformation and can work with less information about the underlying differential equation constraints. In an iterative process, mixed-integer linear programs and small nonlinear programs are solved alternately and the correct and finite terminations are proven. The developments allow to solve stationary gas transport optimization problems with ordinary differential equations. In this sense, promising numerical results for the Greek natural gas transport network are shown.

Second, an instantaneous control algorithm for transient gas network optimization with partial differential equations is presented. A new and specific discretization scheme that allows to use mixed-integer linear programs is developed for the example of gas. Again, promising numerical results that illustrate the applicability of the approach are shown.

About the author:

Mathias Sirvent received his PhD from FAU Erlangen-Nürnberg in March 2018. From 2013 to 2018, he was a research fellow at the economics, discrete optimization, and mathematics (EDOM) group headed by Prof. Dr. Alexander Martin. During that time, the author of this thesis was working on academic and industry-driven projects such as the SFB Transregio 154 about mathematical modeling, simulation, and optimization using the example of gas networks. His research is focused on mixed-integer (non-)linear optimization and operations research.

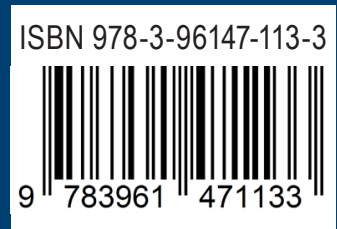

